Geometry $\&$ Topology

Volume 9 (2005) 833-934

Published: 23 May 2005

Revised: 13 December 2005 (see footnote 3 on page 834)

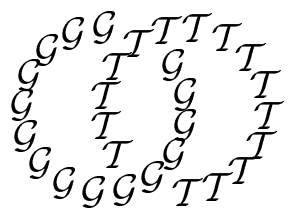

\title{
Bar constructions for topological operads and the Goodwillie derivatives of the identity
}

\author{
Michael Ching \\ Department of Mathematics, Room 2-089 \\ Massachusetts Institute of Technology \\ Cambridge, MA 02139, USA \\ Email: mcching@math.mit.edu
}

\begin{abstract}
We describe a cooperad structure on the simplicial bar construction on a reduced operad of based spaces or spectra and, dually, an operad structure on the cobar construction on a cooperad. We also show that if the homology of the original operad (respectively, cooperad) is Koszul, then the homology of the bar (respectively, cobar) construction is the Koszul dual. We use our results to construct an operad structure on the partition poset models for the Goodwillie derivatives of the identity functor on based spaces and show that this induces the 'Lie' operad structure on the homology groups of these derivatives. We also extend the bar construction to modules over operads (and, dually, to comodules over cooperads) and show that a based space naturally gives rise to a left module over the operad formed by the derivatives of the identity.
\end{abstract}

\section{AMS Classification numbers Primary: $55 \mathrm{P} 48$}

Secondary: 18D50, 55P 43

Keywords: Operad, cooperad, bar construction, module

Proposed: Thomas Goodwillie

Seconded: Ralph Cohen, Gunnar Carlsson
Received: 18 March 2005

Accepted: 6 May 2005 


\section{Introduction}

The motivation for this paper was an effort to construct an operad structure on the derivatives (in the sense of Tom Goodwillie's homotopy calculus [10, 11, 12]) of the identity functor $I$ on the category of based spaces. Such an operad structure has been 'known' intuitively by experts for some time but, as far as the author knows, no explicit construction has previously been given. One piece of evidence for such a structure is the calculation, due to various people, of the homology of these derivatives. This homology is the suspension of the standard Lie operad and so is itself an operad. It is reasonable to ask, therefore, if there is an operad structure on the derivatives themselves that induces this structure on the homology.

Our construction is based on the partition poset model for the derivatives $\partial_{*} I$ described by Arone and Mahowald in [1. They show that the derivatives are the dual spectra associated to certain finite complexes known as the partition poset complexes. In the present work we notice that these complexes are precisely the simplicial bar construction 2 on the operad $P$ in based spaces with $P(n)=$ $S^{0}$ for all $n$. Most of the paper is concerned with showing that such a bar construction has a natural cooperad structure 3 We do this by reinterpreting the bar construction in terms of spaces of trees. The cooperad structure then comes from a natural way to break trees apart. Taking duals, we get the required operad structure on the derivatives of the identity. In fact, we can view the derivatives of the identity as a cobar construction on the cooperad $Q$ in spectra with $Q(n)=S$, the sphere spectrum, for all $n$.

In the final part of the paper (Section 9) we show that by taking homology we do indeed recover the 'Lie' operad structure on $H_{*}\left(\partial_{*} I\right)$. We do this by introducing spectral sequences for calculating the homology of the topological bar and cobar constructions. The $E^{1}$ terms of these spectral sequences can be identified with algebraic versions of the bar and cobar constructions, which

\footnotetext{
${ }^{1}$ The Goodwillie derivatives of a homotopy functor are a sequence of spectra with actions by the symmetric groups, but are only defined up to homotopy. By an operad structure on these derivatives, we mean choices of models for these spectra in a suitable symmetric monoidal category, such as the category of $S$-modules of EKMM 6 , together with an operad structure on those models.

${ }^{2}$ See, for example, [16. Section II.2.3] for the general form of the two-sided simplicial bar construction.

${ }^{3}$ After this paper was written, the author learnt that this result had already been proved in unpublished work of Salvatore [17 using an alternative definition of the bar construction on an operad. See Remark 4.7
} 
in turn are related to the theory of Koszul duality for operads introduced by Ginzburg and Kapranov in [9]. Our main result on this connection is that if the homology of a topological operad $P$ is Koszul, then the homology of the bar construction $B(P)$ is its Koszul dual cooperad. In our case of interest, we deduce that the induced operad structure on the homology of the derivatives of the identity is that of the Koszul dual of the cocommutative cooperad. This is precisely the 'Lie' operad structure referred to above.

\section{Outline of the paper}

We now give a more detailed description of the paper. The first two sections are concerned with preliminaries. In Section 1 we recall the notions of symmetric monoidal and enriched categories and specify the categories we will be working with in this paper. These are symmetric monoidal categories that are enriched, tensored and cotensored over the category $\mathcal{T}$ of based compactly-generated spaces (where $\mathcal{T}$ is a symmetric monoidal category with respect to the smash product). It is to operads in these categories that we refer in the title when we say 'topological operads'. We also require an extra condition that relates the symmetric monoidal structure to the tensoring over $\mathcal{T}$. This condition (see Definition [1.10) is crucial to our later constructions. The two main examples of categories satisfying our requirements are: based spaces themselves, and a suitable symmetric monoidal category of spectra, such as that of EKMM [6].

In Section 2 we recall the definitions of operads and cooperads. We should stress that the constructions of this paper apply only to what we call reduced operads and cooperads. These are $P$ with $P(0)=*$ and $P(1)=S$ the unit of the symmetric monoidal structure. The bar construction can still be defined for more general operads, but the cooperad structure described here does not seem to extend to such cases. In this section we also define modules and comodules over operads and cooperads respectively.

The real substance of the paper starts in Section 3. Here we define the trees that will form the combinatorial heart of our description of the bar and cobar constructions. It is not a coincidence that these trees are the same species used by, for example, Getzler and Jones in their work [8] on the bar constructions for algebraic operads and Koszul duality. We also describe what we call a weighting on a tree (Definition 3.7), that is, a suitable assignment of lengths to the edges of the tree. The spaces $w(T)$ of weightings are at the heart of everything we do in this paper.

In Section 4.1 we give our description of the bar construction on an operad in terms of such trees. If $P$ is an operad of based spaces, we can think of a 
point in the bar construction $B(P)$ as a weighted tree (that is, a tree with lengths assigned to the edges) with vertices labelled by points coming from the spaces $P(n)$. See Definition 4.1 for a precise statement and Definition 4.4 for a more formal approach. In Section 4.2 we show that what we have defined is isomorphic to the standard simplicial bar construction on an operad.

In Section 4.3 we concern ourselves with the cooperad structure on $B(P)$. This is given by the process of 'ungrafting' trees (see Definition 4.14 and beyond). This involves taking a weighted, labelled tree and breaking it up into smaller trees. Finding the right way to weight and label these smaller trees gives us the required cooperad structure maps.

One of the advantages of the way we have set up the theory is that the cobar construction on a cooperad is strictly dual to the bar construction on an operad. In Section 5 we go through the definitions and results dual to those of Section 4 .

The short section Section [6 is devoted to a simple but key result (Proposition 6.4) that relates the bar and cobar constructions via a duality functor that reduces to Spanier-Whitehead duality in the case of spectra. This result says that, under the right circumstances, the dual of the bar construction on an operad $P$ is isomorphic to the cobar construction on the dual of $P$. This allows us, later on, to identify the derivatives of the identity as the cobar construction on a cooperad of spectra.

Before turning to our main example and application, we deal in Section 7 with the two-sided bar and cobar constructions. These include the bar construction for a module over an operad and, dually, the cobar construction for a comodule over a cooperad. To describe these requires a fairly simple generalization of

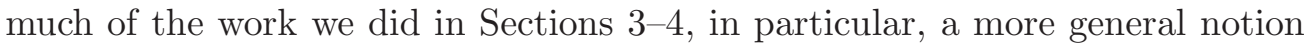
of tree (see Definition [7.1).

Finally, in Section 8 we are able to complete the main aim of this paper. We identify the partition poset complexes with a bar construction and deduce the existence of an operad structure on the derivatives of the identity functor (Corollary 8.8). We also give examples of modules over the resulting operad, including, in particular, a module $M_{X}$ naturally associated to a based space $X$.

The last section of the paper Section 9 is concerned with the relationship of our work to the algebraic bar construction and Koszul operads. As promised, we construct a spectral sequence (Proposition 9.39) relating the two and deduce the result on Koszul duality (Proposition 9.48). 


\section{Future Work}

The work of this paper raises various questions that seem to the author to warrant further attention:

- What is the homotopy theory of the topological bar and cobar constructions? In particular, how do they relate to known model structures on the categories of operads and cooperads (see, for example, Berger-Moerdijk [2])?

- Is there a deeper relationship between Goodwillie's homotopy calculus and the theory of operads? The present paper does not do any calculus, the only connection being via the partition poset complexes. One might ask, for example, if the derivatives of other functors can be described and/or treated using these ideas.

- What object is described by an algebra or module over the operad formed by the derivatives of the identity? In Remark 8.10 we show that a based space $X$ gives rise to such a module. How much of (the homotopy theory of) the space $X$ is retained by this module?

\section{Acknowledgements}

The work of this paper forms the author's PhD thesis written at the Massachusetts Institute of Technology under the supervision of Haynes Miller, to whom the greatest thanks are due for his constant support, encouragement and advice. The idea that the derivatives of the identity might be related to a cobar construction was suggested by work of Kristine Bauer, Brenda Johnson and Jack Morava. The observation that the partition poset complexes (and hence the derivatives of the identity) can be described in terms of spaces of trees was mentioned to the author by Tom Goodwillie, who heard it from Greg Arone. The work of Benoit Fresse [7] on the algebraic side of the theory was invaluable to the present paper. The author has also benefited greatly from conversations with Mark Behrens and Andrew Mauer-Oats while writing this paper, and finally would like to thank the referee for some helpful comments and suggestions.

\section{Symmetric monoidal and enriched categories}

On the one hand, the bar and cobar constructions are most easily defined (and understood) in the category of based spaces. On the other hand, our 
main application is in a category of spectra. We will develop the theory in a general setting that encompasses both cases. This approach will also allow us to appreciate more readily the duality between the bar and cobar constructions.

In this section we recall the basic theory of symmetric monoidal and enriched categories (see [3, Section 6] for a detailed account). We state precisely (Definition 1.10) the structure we will require of a category to make the bar and cobar constructions in it. The only material in this chapter that is not standard is the definition of enriched symmetric monoidal categories or 'symmetric monoidal $\mathcal{V}$-categories' as we have called them (Definition 1.10). The 'distributivity' morphism described there is a key component of the constructions made later in the paper and so we draw the reader's attention to it now.

Definition 1.1 (Symmetric monoidal categories) A monoidal category consists of

- a (locally small) category $\mathcal{V}$,

- a functor $-\wedge-: \mathcal{V} \times \mathcal{V} \rightarrow \mathcal{V}$,

- a unit object $I$ in $\mathcal{V}$ together with natural isomorphisms $X \wedge I \cong X \cong$ $I \wedge X$,

- a natural associativity isomorphism $X \wedge(Y \wedge Z) \cong(X \wedge Y) \wedge Z$,

such that the appropriate three coherence diagrams commute [15, Section VII]. A symmetric monoidal category is a monoidal category together with

- a natural symmetry isomorphism $X \wedge Y \cong Y \wedge X$,

such that four additional coherence diagrams also commute. We will denote such a symmetric monoidal category by $(\mathcal{V}, \wedge, I)$, or just $\mathcal{V}$ with the rest of the structure understood.

Remark 1.2 We will not give names to the associativity and symmetry isomorphisms in a symmetric monoidal category. When we write unbracketed expressions such as

$$
X \wedge Y \wedge Z
$$

or unordered expressions such as

$$
\bigwedge_{a \in A} X_{a}
$$

we mean any one particular choice of ordering and bracketing. Different choices are related by the appropriate associativity and commutativity isomorphisms between them. A map to or from a particular choice determines a map to or from any other choice by composing with the relevant isomorphism. 
Definition 1.3 A closed symmetric monoidal category is a symmetric monoidal category $(\mathcal{V}, \wedge, I)$ together with a functor

$$
\mathcal{V}^{\mathrm{op}} \times \mathcal{V} \rightarrow \mathcal{V} ;(X, Y) \mapsto \operatorname{Map}(X, Y)
$$

and a natural isomorphism of sets

$$
\operatorname{Hom}_{\mathcal{V}}(X \wedge Y, Z) \cong \operatorname{Hom}_{\mathcal{V}}(X, \operatorname{Map}(Y, Z)),
$$

where $\operatorname{Hom}_{\mathcal{V}}(X, Y)$ is the set of morphisms from $X$ to $Y$ in the category $\mathcal{V}$.

Remark 1.4 The natural isomorphism of sets in Definition 1.3 can be made into an isomorphism within $\mathcal{V}$. That is, in any closed symmetric monoidal category there is a natural isomorphism

$$
\operatorname{Map}(X \wedge Y, Z) \cong \operatorname{Map}(X, \operatorname{Map}(Y, Z)) .
$$

See [3, Section 6.5.3] for details.

Definition 1.5 (Enriched categories) Let $(\mathcal{V}, \wedge, I)$ be a given closed symmetric monoidal category. A $\mathcal{V}$-category or category enriched over $\mathcal{V}$ consists of

- a class $\mathcal{C}$,

- for each pair of elements $C, D \in \mathcal{C}$, an object $\operatorname{Map}_{\mathcal{V}}(C, D)$ of $\mathcal{V}$,

- composition morphisms

$$
\operatorname{Map}_{\mathcal{V}}(C, D) \wedge \operatorname{Map}_{\mathcal{V}}(D, E) \rightarrow \operatorname{Map}_{\mathcal{V}}(C, E)
$$

for each $C, D, E \in \mathcal{C}$,

- identity morphisms

$$
I \rightarrow \operatorname{Map}_{\mathcal{V}}(C, C)
$$

for each $C \in \mathcal{C}$,

that satisfy the appropriate conditions [3, Section 6.2.1]. We will denote such a $\mathcal{V}$-category by $\mathcal{C}$ with the rest of the structure understood.

Remark 1.6 We include some basic observations about enriched categories from [3. Section 6.2].

(1) Let $($ Set, $\times, *)$ be the symmetric monoidal category of sets under cartesian product. A Set-category is then the same thing as a (locally small) category. 
(2) A $\mathcal{V}$-category $\mathcal{C}$ has an underlying category whose objects are the elements of $\mathcal{C}$ and whose morphisms $C \rightarrow D$ are the elements of the set $\operatorname{Hom}_{\mathcal{V}}\left(I, \operatorname{Map}_{\mathcal{V}}(C, D)\right)$, where $I$ is the unit object of $\mathcal{V}$. We often therefore think of a $\mathcal{V}$-category $\mathcal{C}$ as a normal category with extra structure given by the objects $\operatorname{Map}_{\mathcal{V}}(C, D)$.

(3) A closed symmetric monoidal category $\mathcal{V}$ is enriched over itself with

$$
\operatorname{Map}_{\mathcal{V}}(X, Y):=\operatorname{Map}(X, Y) .
$$

Definition 1.7 (Tensoring and cotensoring) Let $\mathcal{C}$ be a $\mathcal{V}$-category. A tensoring of $\mathcal{C}$ over $\mathcal{V}$ is a functor

$$
\mathcal{V} \times \mathcal{C} \rightarrow \mathcal{C} ;(X, C) \mapsto X \otimes C
$$

together with a natural isomorphism

$$
\operatorname{Map}_{\mathcal{V}}(X \otimes C, D) \cong \operatorname{Map}\left(X, \operatorname{Map}_{\mathcal{V}}(C, D)\right) .
$$

A category $\mathcal{C}$ tensored over $\mathcal{V}$ is a $\mathcal{V}$-category together with a chosen tensoring. A cotensoring of $\mathcal{C}$ over $\mathcal{V}$ is a functor

$$
\mathcal{V}^{\text {op }} \times \mathcal{C} \rightarrow \mathcal{C} ;(X, D) \mapsto \operatorname{Map}_{\mathcal{C}}(X, D)
$$

together with a natural isomorphism

$$
\operatorname{Map}_{\mathcal{V}}\left(C, \operatorname{Map}_{\mathcal{C}}(X, D)\right) \cong \operatorname{Map}\left(X, \operatorname{Map}_{\mathcal{V}}(C, D)\right) .
$$

A category $\mathcal{C}$ cotensored over $\mathcal{V}$ is a $\mathcal{V}$-category together with a chosen cotensoring.

Remark 1.8 Here are some basic observations about tensorings and cotensorings.

(1) A closed symmetric monoidal category $(\mathcal{V}, \wedge, I)$ is tensored and cotensored over itself with $X \otimes Y:=X \wedge Y$ and $\operatorname{Map}_{\mathcal{V}}(X, Y):=\operatorname{Map}(X, Y)$.

(2) If $\mathcal{C}$ is tensored over $\mathcal{V}$, we have natural isomorphisms

$$
(X \wedge Y) \otimes C \cong X \otimes(Y \otimes C)
$$

for $X, Y \in \mathcal{V}$ and $C \in \mathcal{C}$. If $\mathcal{C}$ is cotensored over $\mathcal{V}$, we have natural isomorphisms

$$
\operatorname{Map}_{\mathcal{C}}(X \wedge Y, C) \cong \operatorname{Map}_{\mathcal{C}}\left(X, \operatorname{Map}_{\mathcal{C}}(Y, C)\right)
$$

for $X, Y \in \mathcal{V}$ and $C \in \mathcal{C}$. 
Proposition 1.9 Let $\mathcal{C}$ be a $\mathcal{V}$-category. Then $\mathcal{C}^{o p}$ has a natural enrichment over $\mathcal{V} \mathbb{U}$ If $\mathcal{C}$ is tensored, then $\mathcal{C}^{o p}$ is naturally cotensored and vice versa.

Proof We define an enrichment on $\mathcal{C}^{\text {op }}$ by

$$
\operatorname{Map}_{\mathcal{V}}\left(C^{\text {op }}, D^{\text {op }}\right):=\operatorname{Map}_{\mathcal{V}}(D, C)
$$

where $C^{\text {op }}$ is the object in $\mathcal{C}^{\text {op }}$ corresponding to $C \in \mathcal{C}$. If $-\otimes-$ is a tensoring for $\mathcal{C}$ then we get a cotensoring for $\mathcal{C}^{\text {op }}$ by setting

$$
\operatorname{Map}_{\mathcal{C}^{\mathrm{op}}}\left(X, D^{\mathrm{op}}\right):=(X \otimes D)^{\mathrm{op}} .
$$

The required natural isomorphism comes from

$$
\begin{aligned}
\operatorname{Map}_{\mathcal{V}}\left(C^{\text {op }}, \operatorname{Map}_{\mathcal{C}^{\text {op }}}\left(X, D^{\text {op }}\right)\right) & =\operatorname{Map}_{\mathcal{V}}(X \otimes D, C) \\
& \cong \operatorname{Map}\left(X, \operatorname{Map}_{\mathcal{V}}(D, C)\right) \\
& =\operatorname{Map}\left(X, \operatorname{Map}_{\mathcal{V}}\left(C^{\text {op }}, D^{\text {op }}\right)\right)
\end{aligned}
$$

The vice versa part is similar.

We are interested in categories that both are themselves symmetric monoidal categories and are enriched over another symmetric monoidal category. The following definition contains the properties of these that we require in this paper.

Definition 1.10 Let $(\mathcal{V}, \wedge, I)$ be a closed symmetric monoidal category. A symmetric monoidal $\mathcal{V}$-category consists of

- a symmetric monoidal category $(\mathcal{C}, \bar{\wedge}, S)$ with $\mathcal{C}$ enriched, tensored and cotensored over $\mathcal{V}$,

- a natural transformation

$$
d:(X \wedge Y) \otimes(C \bar{\wedge} D) \rightarrow(X \otimes C) \bar{\wedge}(Y \otimes D)
$$

satisfying the following axioms:

- (Associativity) The diagram

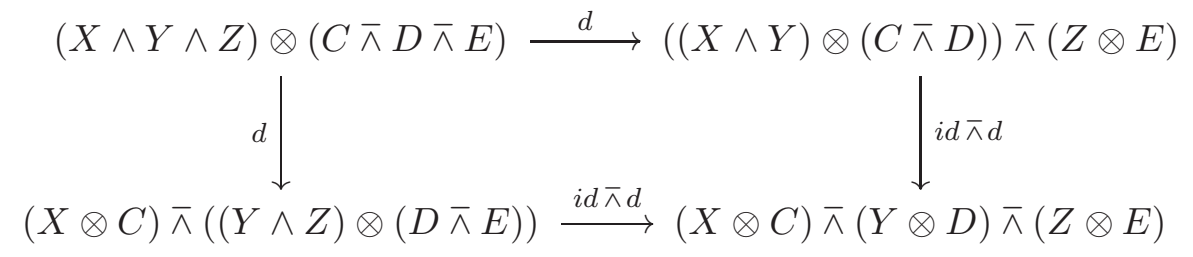

commutes for all $X, Y, Z \in \mathcal{V}$ and $C, D, E \in \mathcal{C}$.

\footnotetext{
${ }^{4}$ Here $\mathcal{C}^{\text {op }}$ denotes the opposite category of the category underlying $\mathcal{C}$ described in Remark 1.6 (2).
} 
- (Unit) The composite

$$
X \otimes C \cong(X \wedge I) \otimes(C \bar{\wedge} S) \stackrel{d}{\longrightarrow}(X \otimes C) \bar{\wedge}(I \otimes S) \cong X \otimes C
$$

is the identity, for any $X \in \mathcal{V}$ and $C \in \mathcal{C}$. Recall that $I, S$ are the units of the symmetric monoidal structures on $\mathcal{V}, \mathcal{C}$ respectively.

The transformation $d$ (for 'distribute') is our way of relating the symmetric monoidal structures in the two categories. It will be essential in constructing the cooperad structure on the bar construction of an operad (see Definition 4.26).

Remark 1.11 A closed symmetric monoidal category $\mathcal{V}$ is itself a symmetric monoidal $\mathcal{V}$-category with the transformation $d$ given by the symmetry and associativity isomorphism:

$$
(X \wedge Y) \wedge(C \wedge D) \cong(X \wedge C) \wedge(Y \wedge D)
$$

Proposition 1.12 Let $\mathcal{C}$ be a symmetric monoidal $\mathcal{V}$-category. Then $\mathcal{C}^{o p}$ is naturally also a symmetric monoidal $\mathcal{V}$-category.

Proof We already know from Proposition 1.9 that $\mathcal{C}^{\text {op }}$ is enriched, tensored and cotensored over $\mathcal{V}$ and there is a canonical symmetric monoidal structure on $\mathcal{C}^{\text {op }}$ given by that on $\mathcal{C}$. It therefore only remains to construct the map $d$. The tensoring in $\mathcal{C}^{\mathrm{op}}$ is given by the cotensoring in $\mathcal{C}$. Therefore $d$ for $\mathcal{C}^{\mathrm{op}}$ corresponds to the following map in $\mathcal{C}$ :

$$
\operatorname{Map}_{\mathcal{C}}(X, C) \wedge \operatorname{Map}_{\mathcal{C}}(Y, D) \rightarrow \operatorname{Map}_{\mathcal{C}}(X \wedge Y, C \bar{\wedge} D)
$$

This is adjoint to a map

$$
(X \wedge Y) \otimes\left(\operatorname{Map}_{\mathcal{C}}(X, C) \bar{\wedge} \operatorname{Map}_{\mathcal{C}}(Y, D)\right) \rightarrow C \bar{\wedge} D
$$

constructed by first using $d$ for $\mathcal{C}$ to get to

$$
\left(X \otimes \operatorname{Map}_{\mathcal{C}}(X, C)\right) \bar{\wedge}\left(Y \otimes \operatorname{Map}_{\mathcal{C}}(Y, D)\right)
$$

and then using the evaluation maps

$$
X \otimes \operatorname{Map}_{\mathcal{C}}(X, C) \rightarrow C \text { and } Y \otimes \operatorname{Map}_{\mathcal{C}}(Y, D) \rightarrow D .
$$

An important property of the categories that we work with in this paper is that they are pointed, that is, they have a null object $*$ that is both initial and terminal. The following proposition describes how null objects interact with symmetric monoidal structures and enrichments. 
Proposition 1.13 Let $(\mathcal{V}, \wedge, I)$ be a closed symmetric monoidal category that is pointed with null object $*$. Then

$$
* \wedge X \cong * \cong \operatorname{Map}(*, X) \cong \operatorname{Map}(X, *)
$$

for all $X \in \mathcal{V}$.

Moreover, let $\mathcal{C}$ be a category enriched over $\mathcal{V}$. If $\mathcal{C}$ is tensored then $* \otimes C$ is an initial object in $\mathcal{C}$ for all $C \in \mathcal{C}$. If $\mathcal{C}$ is cotensored then $\operatorname{Map}_{\mathcal{C}}(*, D)$ is a terminal object in $\mathcal{C}$ for all $D \in \mathcal{C}$.

Finally, if $\mathcal{C}$ is both tensored and cotensored over $\mathcal{V}$, then the initial and terminal objects are isomorphic and so $\mathcal{C}$ is itself pointed.

Proof We observe that

$$
\operatorname{Hom}_{\mathcal{V}}(* \wedge X, Y) \cong \operatorname{Hom}_{\mathcal{V}}(*, \operatorname{Map}(X, Y))
$$

which has one element for any $X, Y$. This tells us that $* \wedge X$ is initial and hence isomorphic to $*$. The other isomorphisms in the first part of the proposition are similar.

Next, the tensoring functor $-\otimes C: \mathcal{V} \rightarrow \mathcal{C}$ is a left adjoint so preserves an initial object. Dually, the cotensoring functor $\operatorname{Map}_{\mathcal{C}}(-, D): \mathcal{V}^{\text {op }} \rightarrow \mathcal{C}$ is a right adjoint so preserves the terminal object. This gives us the second part.

Finally, if $\mathcal{C}$ is both tensored and cotensored, we get a map from the terminal object to the initial object by

$$
\operatorname{Map}_{\mathcal{C}}(*, D) \rightarrow I \otimes \operatorname{Map}_{\mathcal{C}}(*, D) \rightarrow * \otimes \operatorname{Map}_{\mathcal{C}}(*, D)
$$

The first map here is an example of a general isomorphism $C \rightarrow I \otimes C$ where $I$ is the unit object of $\mathcal{V}$. The second map comes from $I \rightarrow *$. A map from a terminal object to an initial object must be an isomorphism. Therefore $\mathcal{C}$ is pointed.

Examples 1.14 The categories with which we will mainly be concerned in this paper are the following.

(1) Let $\mathcal{T}$ be the category of compactly generated based spaces and basepointpreserving continuous maps of [14]. Then $\mathcal{T}$ is a pointed closed symmetric monoidal category under the usual smash product $\wedge$, with unit the 0 sphere $S^{0}$ and $\operatorname{Map}(X, Y)$ equal to the space of basepoint-preserving maps $X \rightarrow Y$. 
(2) Let $\mathcal{S} p$ be the category of $S$-modules of EKMM [6]. Then $\left(\mathcal{S} p, \wedge_{S}, S\right)$ is a symmetric monoidal $\mathcal{T}$-category, where $S$ is the sphere spectrum and $\wedge_{S}$ is the smash product of $S$-modules [6, Section II.1.1]. The enrichment, tensoring and cotensoring are described in [6, Section VII.2.8]. For the distributivity map $d$ we have a natural isomorphism

$$
d:(X \wedge Y) \wedge(E \wedge S F) \stackrel{\cong}{\longrightarrow}(X \wedge E) \wedge S(Y \wedge F)
$$

given by the fact that $X \wedge E \cong(X \wedge S) \wedge_{S} E$ (see [6, Section II.1.4]).

We will usually work with a general symmetric monoidal $\mathcal{T}$-category denoted $(\mathcal{C}, \bar{\wedge}, S)$, but these examples will be foremost in our minds.

\section{Operads and cooperads}

In this section $(\mathcal{C}, \bar{\wedge}, S)$ denotes a pointed symmetric monoidal category with null object $*$. We will assume that $\mathcal{C}$ has all necessary limits and colimits and write the coproduct in $\mathcal{C}$ as a wedge product using $\vee$.

Definition 2.1 (Symmetric sequences) A symmetric sequence in $\mathcal{C}$ is a functor $F$ from the category of nonempty finite sets and bijections to $\mathcal{C}$. For each nonempty finite set $A$, the symmetric group $\Sigma_{A}$ acts on $F(A)$. We will write $F(n)$ for $F(\{1, \ldots, n\})$. Note that our symmetric sequences (and hence our operads) do not have an $F(0)$ term because our indexing sets are nonempty. We will often write 'finite set' when we mean 'nonempty finite set' and these will usually be labelled $A, B, \ldots$ We write $\mathcal{C}^{\Sigma}$ for the category of symmetric sequences in $\mathcal{C}$ (whose morphisms are the natural transformations).

There are several different but equivalent ways to define operads (see MarklShnider-Stasheff [16] for a comprehensive guide). We will use the following definition.

Definition 2.2 (Operads) An operad in the symmetric monoidal category $(\mathcal{C}, \bar{\wedge}, S)$ is a symmetric sequence $P$ together with partial composition maps

$$
-\circ_{a}-: P(A) \bar{\wedge} P(B) \rightarrow P\left(A \cup \cup_{a} B\right)
$$

for each pair of finite sets $A, B$, and each $a \in A$ (where $A \cup_{a} B$ denotes $(A \backslash\{a\}) \amalg B)$, and a unit map

$$
\eta: S \rightarrow P(1)
$$

The composition maps must be natural in $A$ and $B$ and must satisfy the following four axioms: 
(1) The diagram

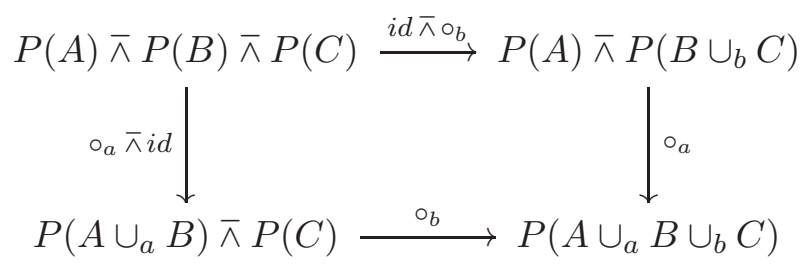

commutes for all $a \in A$ and $b \in B$. (Notice that $\left(A \cup_{a} B\right) \cup_{b} C=$ $\left.A \cup_{a}\left(B \cup_{b} C\right).\right)$

(2) The diagram

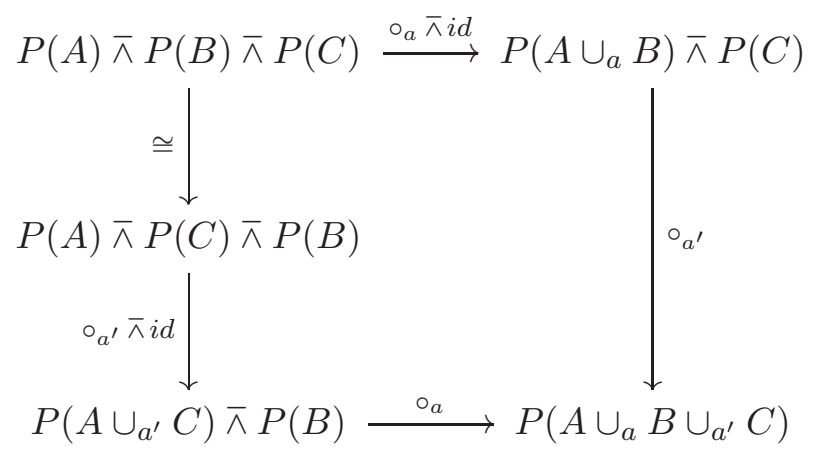

commutes for all $a \neq a^{\prime} \in A$. (Notice that $\left.\left(A \cup_{a} B\right) \cup_{a^{\prime}} C=\left(A \cup_{a^{\prime}} C\right) \cup_{a} B.\right)$

(3) The diagram

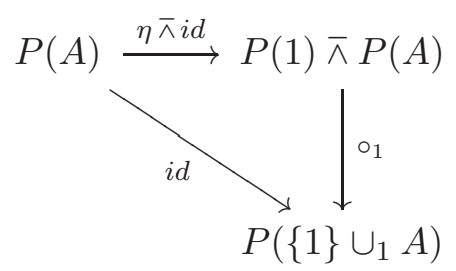

commutes for all $A$.

(4) The diagram

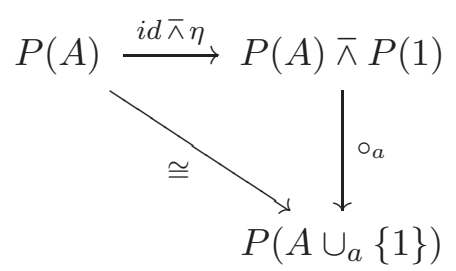

commutes for all $a \in A$. (The diagonal map here is induced by the obvious bijection $A \rightarrow A \cup_{a}\{1\}$.) 
A morphism of operads $P \rightarrow P^{\prime}$ is a morphism of symmetric sequences that commutes with the composition and unit maps.

Definition 2.3 An augmentation of an operad $P$ is a map $\varepsilon: P(1) \rightarrow S$ such that the composite

$$
S \stackrel{\eta}{\longrightarrow} P(1) \stackrel{\varepsilon}{\longrightarrow} S
$$

is the identity on $S$. An augmented operad is an operad together with an augmentation. An operad $P$ is reduced if the unit map $\eta: S \rightarrow P(1)$ is an isomorphism. A reduced operad has a unique augmentation given by the inverse of the unit map. A morphism of augmented operads is a morphism of operads that commutes with the augmentation.

Remark 2.4 Operads are a generalization of monoids for the symmetric monoidal category $(\mathcal{C}, \bar{\wedge}, S)$. A monoid $X$ in $\mathcal{C}$ gives rise to an operad $P_{X}$ with $P_{X}(1)=X$ and $P_{X}(n)=*$ for $n>1$. Conversely, given an operad $P$ in the symmetric monoidal category $\mathcal{C}, P(1)$ is a monoid in $\mathcal{C}$.

An alternative definition of an operad is based on a monoidal structure on the category of symmetric sequences. We define this monoidal structure now.

Definition 2.5 (Composition product of symmetric sequences) Let the composition product of the two symmetric sequences $M, N$ be the symmetric sequence $M \circ N$ with

$$
(M \circ N)(A):=\bigvee_{A=\coprod_{j \in J} A_{j}} M(J) \bar{\wedge} \bigwedge_{j \in J} N\left(A_{j}\right) .
$$

The coproduct here is taken over all unordered partitions of $A$ into a collection of nonempty subsets $\left\{A_{j}\right\}_{j \in J}$. The particular choice of indexing set is not important in the sense that we do not sum over different $J$ that index the same partition. A bijection $A \rightarrow A^{\prime}$ determines a bijection between partitions of $A$ and partitions of $A^{\prime}$ in an obvious way. Thus we match up the terms in the coproducts that define $(M \circ N)(A)$ and $(M \circ N)\left(A^{\prime}\right)$. If $J$ and $J^{\prime}$ index two corresponding partitions of $A$ and $A^{\prime}$ respectively, then we get a natural choice of bijection $J \rightarrow J^{\prime}$. Moreover, if $j \in J$ and $j^{\prime} \in J^{\prime}$ correspond under this bijection then we get a bijection $A_{j} \rightarrow A_{j^{\prime}}^{\prime}$ by restricting the bijection $A \rightarrow A^{\prime}$. The actions of $M$ and $N$ on these bijections together give us an isomorphism

$$
(M \circ N)(A) \rightarrow(M \circ N)\left(A^{\prime}\right) .
$$

Thus $M \circ N$ becomes a symmetric sequence in $\mathcal{C}$. 
Definition 2.6 The unit symmetric sequence in the pointed symmetric monoidal category $(\mathcal{C}, \bar{\wedge}, S)$ is the symmetric sequence $I$ given by

$$
I(A):= \begin{cases}S & \text { if }|A|=1 \\ * & \text { otherwise }\end{cases}
$$

where $*$ is the null object of $\mathcal{C}$.

Lemma 2.7 Let $(\mathcal{C}, \bar{\wedge}, S)$ be a pointed symmetric monoidal category. Then for any symmetric sequence $M$ there are natural isomorphisms

$$
M \circ I \cong M \cong I \circ M
$$

Proof For the finite set $A$, the only term that contributes to $(M \circ I)(A)$ comes from the partition of $A$ into singleton subsets. This makes it clear that $M \circ I \cong M$. The only term that contributes to $(I \circ M)(A)$ comes from the trivial partition of $A$ into one subset, that is $A$ itself. From this we see that $I \circ M \cong M$.

To get a monoidal structure on the category of symmetric sequences, we also need an associativity isomorphism. This does not exist in general, although it does in the case of the following lemma.

Lemma 2.8 Let $(\mathcal{C}, \bar{\wedge}, S)$ be a pointed symmetric monoidal category in which $\bar{\wedge}$ commutes with finite coproducts. Then there are natural isomorphisms

$$
L \circ(M \circ N) \cong(L \circ M) \circ N
$$

for symmetric sequences $L, M, N$ in $\mathcal{C}$.

Proof Using the hypothesis that $\bar{\wedge}$ commutes with finite coproducts, it is not hard to see that each side is naturally isomorphic to the symmetric sequence $(L \circ M \circ N)$ given by

$$
(L \circ M \circ N)(A):=\bigvee_{A=\coprod_{b \in B} A_{b}, B=\coprod_{c \in C} B_{c}} L(C) \bar{\wedge} \bigwedge_{c \in C} M\left(B_{c}\right) \bar{\wedge} \bigwedge_{b \in B} N\left(A_{b}\right) .
$$

The coproduct here is over all partitions of $A$ into nonempty subsets indexed by some set $B$, together with a partition of $B$ into subsets indexed by some $C$. Equivalently, the coproduct is indexed of pairs of partitions of $A$, one (indexed by $B$ ) a refinement of the other (indexed by $C$ ).

The following description of operads is due to Smirnov. See [16, Theorem 1.68] for further details. 
Proposition 2.9 Let $(\mathcal{C}, \bar{\wedge}, S)$ be a pointed symmetric monoidal category in which $\bar{\wedge}$ commutes with finite coproducts. Then the composition product $\circ$ is a monoidal product on the category of symmetric sequences in $\mathcal{C}$ with unit object $I$ and unit and associativity isomorphisms given by Lemmas 2.7 and 2.8 respectively. In this case, an operad in $\mathcal{C}$ is precisely a monoid for this monoidal product.

Proof One can easily check that the axioms for a monoidal structure are satisfied. If $P$ is an operad in $\mathcal{C}$, the operad compositions make up a map

$$
P \circ P \rightarrow P
$$

and the unit map $\eta$ gives a map of symmetric sequences

$$
I \rightarrow P \text {. }
$$

The operad axioms then translate into associativity and unit axioms that give $P$ the structure of a monoid under $\circ$.

Remark 2.10 If $\mathcal{C}$ is a closed symmetric monoidal category then $\bar{\lambda}$ has a right adjoint and so preserves all colimits. In particular, the hypothesis of Lemma 2.8 holds and so we get a true monoidal structure on the symmetric sequences in $\mathcal{C}$.

Unfortunately, even when $\mathcal{C}$ is closed symmetric monoidal, its opposite category $\mathcal{C}^{\text {op }}$ (with the standard symmetric monoidal structure) is unlikely to be closed. Since we will want to dualize most of the results of this paper to be able to deal with cooperads as well as operads, we need to get round this hypothesis. For this, we notice that in general there are natural maps of symmetric sequences

$$
(L \circ M \circ N) \rightarrow L \circ(M \circ N)
$$

and

$$
(L \circ M \circ N) \rightarrow(L \circ M) \circ N
$$

where $(L \circ M \circ N)$ is defined as in the proof of Lemma 2.8. In general these are not isomorphisms so we do not get a monoidal structure on the category of symmetric sequences. However, it is possible to define monoids in this more general case (see [5] for more details), and we get the following alternative characterization of an operad.

Proposition 2.11 Let $(\mathcal{C}, \bar{\wedge}, S)$ be a pointed symmetric monoidal category. An operad in $\mathcal{C}$ is equivalent to a symmetric sequence $P$ together with maps

$$
m: P \circ P \rightarrow P ; \eta: I \rightarrow P
$$

of symmetric sequences such that the following diagrams commute: 
(1) Associativity:

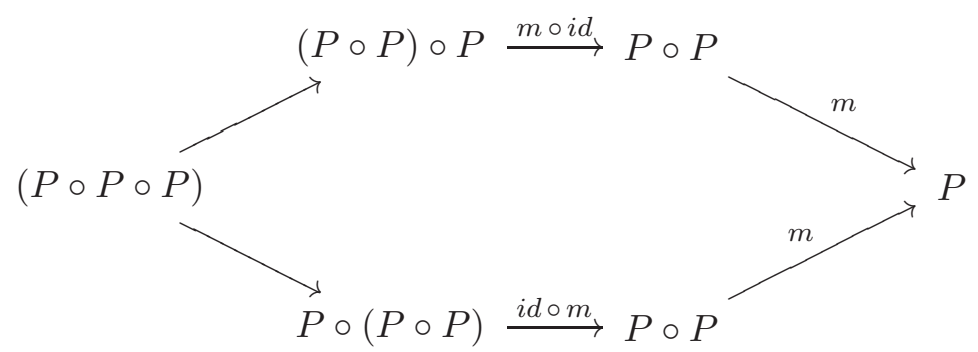

where the two initial arrows are the maps mentioned in Remark 2.10]

(2) Left unit:

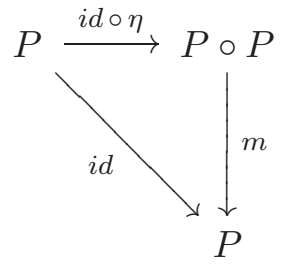

(3) Right unit:

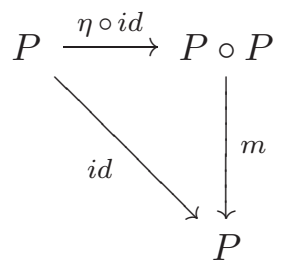

Remark 2.12 We will refer to an operad $P$ as a monoid with respect to the composition product, even when we do not in fact have a monoidal structure. There are similarly defined notions of an object with a right or left action of a monoid in this generalized setting. These give us right and left modules over our operads which we now define.

Definition 2.13 (Modules over operads) A left module over the operad $P$ is a symmetric sequence $M$ together with a left action of the monoid $P$, that is, a map

$$
l: P \circ M \rightarrow M
$$


such that the diagrams

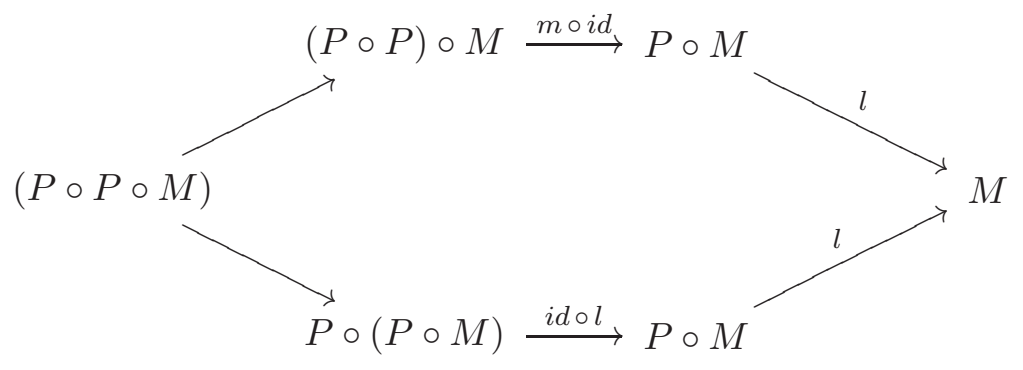

and

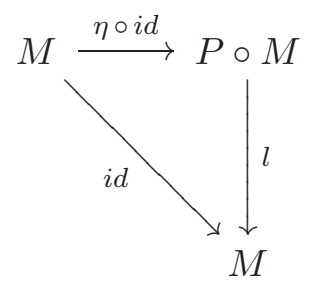

commute.

A right module over $P$ is a symmetric sequence $M$ with a right action of $P$, that is a map

$$
M \circ P \rightarrow M
$$

satisfying corresponding axioms. A $(P, P)$-bimodule is a symmetric sequence $M$ that is both a right and a left module over $P$ such that

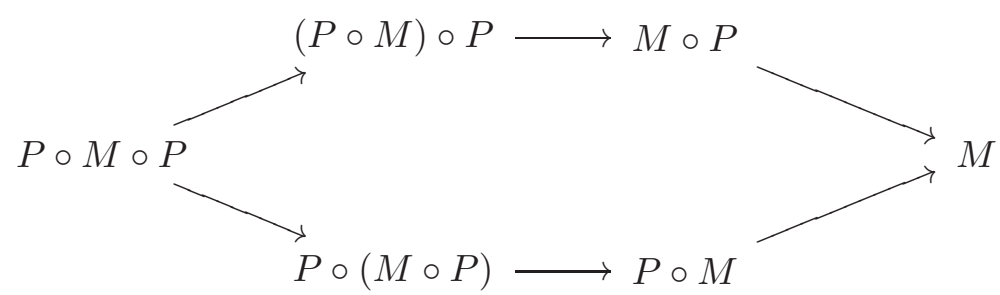

commutes. Clearly, $P$ itself is a $(P, P)$-bimodule.

Remark 2.14 It's useful to have a slightly more explicit description of a module over an operad. The action map for a left $P$-module $M$ consists of maps

$$
P(r) \bar{\wedge} M\left(A_{1}\right) \bar{\wedge} \cdots \bar{\wedge} M\left(A_{r}\right) \rightarrow M(A)
$$

for every partition $A=\coprod_{i=1}^{r} A_{i}$ of a finite set $A$ into nonempty subsets. Conversely, giving maps of this form that satisfy appropriate conditions uniquely determines a left $P$-module. Similarly, a right module structure consists of maps of the form

$$
M(r) \bar{\wedge} P\left(A_{1}\right) \bar{\wedge} \cdots \bar{\wedge} P\left(A_{r}\right) \rightarrow M(A)
$$


Remark 2.15 In the same way that operads are a generalization of monoids in $\mathcal{C}$, modules over those operads are generalization of modules over the monoids. A module $M$ over the monoid $X$ gives rise to a module $P_{M}$ over the operad $P_{X}$ described in Remark 2.4 with $P_{M}(n)=*$ if $n>1$ and $P_{M}(1)=M$.

Remark 2.16 An augmentation for the operad $P$ is equivalent to either a left or right module structure on the unit symmetric sequence $I$.

The standard notion of an algebra over an operad is closely related to that of a module. We briefly describe how this works.

Definition 2.17 (Algebras over an operad) An algebra over the operad $P$ is an object $C \in \mathcal{C}$ together with maps

$$
P(A) \bar{\wedge} \bigwedge_{a \in A} C \rightarrow C
$$

that satisfy appropriate naturality, associativity and unit axioms.

The following result allows us to construct a left $P$-module from a $P$-algebra 5

Lemma 2.18 Let $C$ be an algebra over the operad $P$. Then there is a natural left $P$-module structure on the constant symmetric sequence $\underline{C}$ with $\underline{C}(A)=C$ for all finite sets $A 6$

Proof The components of the module structure map $P \circ \underline{C} \rightarrow \underline{C}$ are given by the algebra structure maps as follows:

$$
P(r) \bar{\wedge} \underline{C}\left(A_{1}\right) \bar{\wedge} \cdots \bar{\wedge} \underline{C}\left(A_{r}\right)=P(r) \bar{\wedge} C^{\bar{\wedge} r} \rightarrow C=\underline{C}(A)
$$

\footnotetext{
${ }^{5}$ There is a more basic way to view algebras over an operad as modules. This requires us to introduce an $M(0)$ term to our modules (that is, our symmetric sequences become functors from the category of all finite sets, not just nonempty finite sets). With a corresponding generalization of the composition product, and hence of the notion of module, a $P$-algebra is equivalent to a left $P$-module concentrated in the $M(0)$ term. The reason we do not allow our modules to have this extra term is that the comodule structure on the bar construction (see Section 7.2) would not then exist in general.

${ }^{6}$ The obvious converse to this Lemma is not true. That is, a constant symmetric sequence together with a left $P$-module structure need not arise from a $P$-algebra. The construction given in the proof of this lemma forces different components of the module structure map to be the same which need not be the same in general.
} 
Definition 2.19 (Cooperads) The notion of a cooperad is dual to that of an operad. That is, a cooperad in $\mathcal{C}$ is an operad in the opposite category $\mathcal{C}^{\text {op }}$ with the canonical symmetric monoidal structure determined by that in $\mathcal{C}$. More explicitly, a cooperad consists of a symmetric sequence $Q$ in $\mathcal{C}$ together with cocomposition maps

$$
Q\left(A \cup_{a} B\right) \rightarrow Q(A) \bar{\wedge} Q(B)
$$

and a counit map

$$
Q(1) \rightarrow S
$$

satisfying axioms dual to (1)-(4) of Definition 2.2. A morphism of cooperads is a morphism of symmetric sequences that commutes with the cocomposition and counit maps. A coaugmentation for a cooperad is a map $S \rightarrow Q(1)$ left inverse to the counit map. A cooperad $Q$ is reduced if the counit map is an isomorphism.

Remark 2.20 The description of an operad as a monoid for the composition product of symmetric sequences naturally dualizes to cooperads. We define the dual composition product $\widehat{o}$ of two symmetric sequences by replacing the coproduct in Definition 2.5 with a product. That is:

$$
M \widehat{\circ} N(A):=\prod_{A=\coprod_{j \in J} A_{j}} M(J) \bar{\wedge} \bigwedge_{j \in J} N\left(A_{j}\right) .
$$

If $\bar{\wedge}$ commutes with finite products (which is in general not likely) this is a monoidal product of symmetric sequences (the result dual to Proposition 2.9) and a cooperad is precisely a comonoid for this product. In general we can define the triple product ( $L \widehat{\circ} M \widehat{o} N$ ) by replacing coproduct with product in the definition given in the proof of Lemma 2.8. We then have natural maps

$$
(L \widehat{\circ} M) \widehat{\circ} N \rightarrow(L \widehat{\circ} M \widehat{\circ} N) \text { and } L \widehat{\circ}(M \widehat{\circ} N) \rightarrow(L \widehat{\circ} M \widehat{\circ} N)
$$

which allow us to say what we mean by a comonoid in general. Thus we get the result dual to Proposition 2.11, that a cooperad in $\mathcal{C}$ is a symmetric sequence $Q$ together with maps

$$
Q \rightarrow Q \widehat{\circ} Q \quad \text { and } \quad Q \rightarrow I
$$

such that the corresponding diagrams commute. In particular we have a coas- 
sociativity diagram:

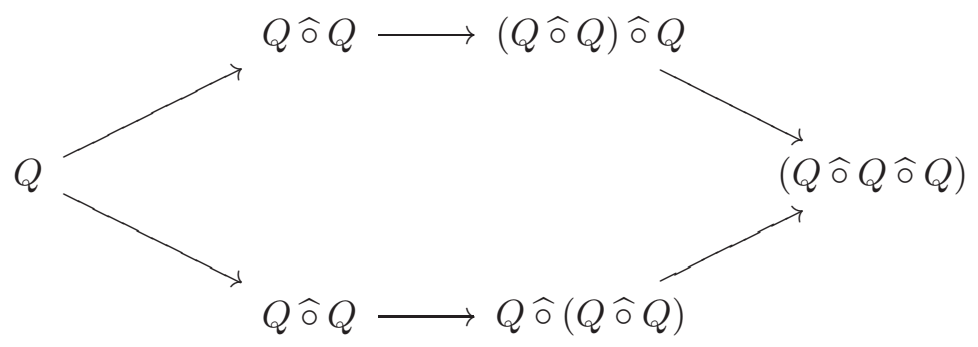

Remark 2.21 In 8] Getzler and Jones define a cooperad to be a comonoid for the composition product $\circ$. In their case, $\circ$ and $\widehat{o}$ are equal because finite products are isomorphic to finite coproducts in the category of chain complexes.

Definition 2.22 (Comodules over a cooperad) A left comodule $C$ over the cooperad $Q$ is a left module over $Q$ considered as an operad in $\mathcal{C}^{\text {op }}$. More explicitly, $C$ is a symmetric sequence together with a left coaction of the comonoid $Q$, that is, a map $C \rightarrow Q \widehat{\circ} C$. Equivalently, we have a suitable collection of cocomposition maps

$$
C(A) \rightarrow Q(r) \bar{\wedge} C\left(A_{1}\right) \bar{\wedge} \cdots \bar{\wedge} C\left(A_{r}\right)
$$

for partitions $A=\coprod_{i=1}^{r} A_{i}$. Similarly a right comodule is a symmetric sequence $C$ with a right coaction $C \rightarrow C \widehat{o} Q$, or equivalently, cocomposition maps

$$
C(A) \rightarrow C(r) \bar{\wedge} Q\left(A_{1}\right) \bar{\wedge} \cdots \bar{\wedge} Q\left(A_{r}\right) .
$$

A bicomodule is a symmetric sequence with compatible left and right comodule structures. The cooperad $Q$ is itself a $(Q, Q)$-bicomodule.

A coalgebra over a cooperad is the dual concept of an algebra over an operad and the constant symmetric sequence with value equal to a $Q$-coalgebra is a left $Q$-comodule.

\section{Spaces of trees}

As mentioned in the introduction to the paper, the key to finding a cooperad structure on the bar construction on an operad is its reinterpretation in terms of trees. These are the same sorts of trees used in many other places to work with operads. See Getzler-Jones [8], Ginzburg-Kapranov [9] and Markl-ShniderStasheff [16] for many examples. 
Definition 3.1 (Trees) A typical tree of the sort we want is shown in Figure 1. It has a root element at the base, a single edge attached to the root, and no other vertices with only one incoming edge. We encode these geometric requirements in the following combinatorial definition. A tree $T$ is a finite poset satisfying the following conditions:

(1) $T$ has at least two elements: an initial (or minimal) element $r$, the root, and another element $b$ such that $b \leq t$ for all $t \in T, t \neq r$.

(2) For any elements $t, u, v \in T$, if $u \leq t$ and $v \leq t$, then either $u \leq v$ or $v \leq u$.

(3) For any $t<u$ in $T$ with $t \neq r$, there is some $v \in T$ such that $t<v$ but $u \not \leq v$.

We picture a tree by its graph, whose vertices are the elements of $T$ with an edge between $t$ and $u$ if $t<u$ and there is no $v$ with $t<v<u$. An incoming edge to a vertex $t$ is an edge corresponding to some relation $t<u$. Condition (1) above ensures that the tree has a root $r$ with exactly one incoming edge (that connects it to $b$ ). The second condition ensures that this graph is indeed a tree in the usual sense. The third condition ensures that no vertices except the root have exactly one incoming edge.

More terminology: the maximal elements of the tree $T$ will be called leaves. From now on, by a vertex, we mean an element other than the root or a leaf (see Figure 1). A tree is binary if each vertex has precisely two incoming edges. The root edge is the edge connected to the root element. The leaf edges are the edges connected to the leaves. The other edges in the tree are internal edges. Given a vertex $v$ of a tree, we write $i(v)$ for the set of incoming edges of the vertex $v$. We generally denote trees with the letters $T, U, \ldots$

Remark 3.2 We stress that our trees are not allowed to have vertices with only one incoming edge, as guaranteed by condition (3) of the definition. This reflects the fact that we will deal only with reduced operads in this paper.

Definition 3.3 (Labellings) A labelling of the tree $T$ by a finite set $A$ is a bijection between $A$ and the set of leaves of $T$. An isomorphism of $A$-labelled trees is an isomorphism of the underlying trees that preserves the labelling. We denote the set of isomorphism classes of $A$-labelled trees by $\mathrm{T}(A)$. For a finite set $A, \mathrm{~T}(A)$ is also finite. For a positive integer $n$, we write $\mathrm{T}(n)$ for the set $\mathrm{T}(\{1, \ldots, n\})$. 


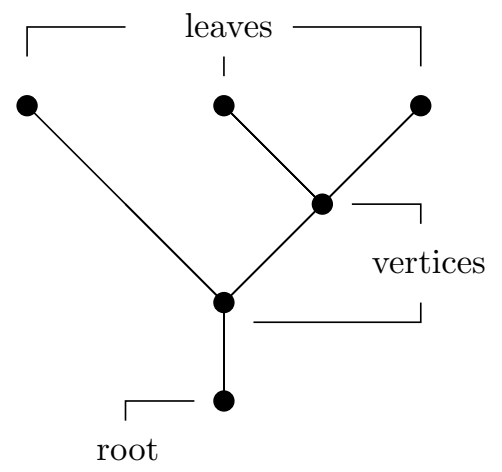

Figure 1: Terminology for trees

Example 3.4 There is up to isomorphism only one tree with one leaf. It has a single edge whose endpoints are the root and the leaf. Thus $\mathrm{T}(1)$ has one element. It is easy to see that $\mathrm{T}(2)$ also only has one element: the tree with one vertex that has two input edges. Figure 2 shows $\mathrm{T}(1), \mathrm{T}(2), \mathrm{T}(3)$.

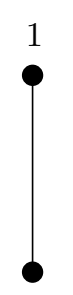

$\mathrm{T}(1)$

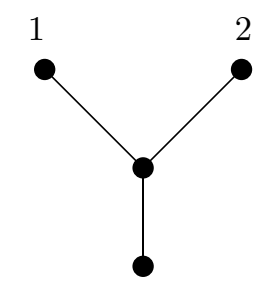

$\mathrm{T}(2)$
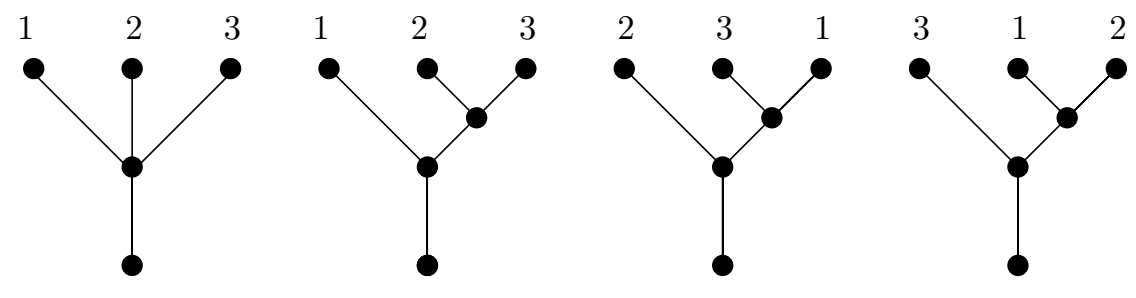

$\mathrm{T}(3)$

Figure 2: Labelled trees with three or fewer leaves 
Definition 3.5 (Edge collapse) Given a tree $T$ and an internal edge $e$, denote by $T / e$ the tree obtained by collapsing the edge $e$, identifying its endpoints. (In poset terms, this is equivalent to removing from the poset the element corresponding to the upper endpoint of the edge.) If $u$ and $v$ are those endpoints, write $u \circ v$ for the resulting vertex of $T / e$. Note that $T / e$ has the same leaves as $T$ so retains any labelling. See Figure 3 for an example.

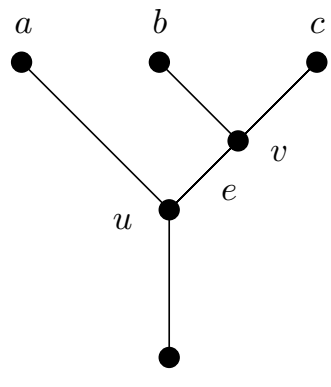

$T$

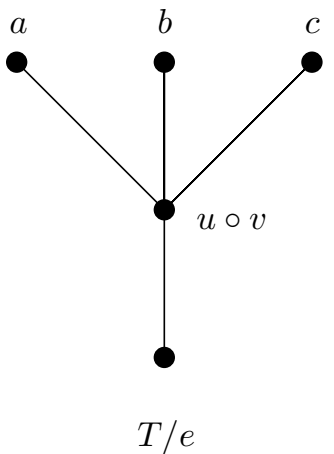

Figure 3: Edge collapse of labelled trees

Definition 3.6 The process of collapsing edges gives us a partial order on the set $\mathrm{T}(A)$ of isomorphism classes of $A$-labelled trees. We say that $T \leq T^{\prime}$ if $T$ can be obtained from $T^{\prime}$ be collapsing a sequence of edges. We think of the resulting poset as a category.

We now give our trees topological significance by introducing 'weightings' on them.

Definition 3.7 A weighting on a tree $T$ is an assignment of nonnegative 'lengths' to the edges of $T$ in such a way that the 'distance' from the root to each leaf is exactly 1 . The set of weightings on a tree $T$ is a subset of the space of functions from the set of edges of $T$ to the unit interval $[0,1]$ and we give it the subspace topology. We denote the resulting space by $w(T)$. A tree together with a weighting is a weighted tree.

Example 3.8 There is only one way to weight the unique tree $T \in \mathrm{T}(1)$ (the single edge must have length 1 ), so $w(T)=*$. For any $n, \mathrm{~T}(n)$ contains a tree $T_{n}$ with a single vertex that has $n$ incoming edges. For this tree we have $w\left(T_{n}\right)=\Delta^{1}$ the topological 1-simplex or unit interval. Figure 2 displays 
another shape of tree with three leaves, one that has two vertices. For such a tree $U$, we have $w(U)=\Delta^{2}$, the topological 2-simplex. Not all spaces of weightings are simplices, but we do have the following result.

Lemma 3.9 Let $T$ be a tree with $n$ (internal) vertices. Then $w(T)$ is homeomorphic to the $n$-dimensional disc $D^{n}$. If $n \geq 1$, the boundary $\partial w(T)$ is the subspace of weightings for which at least one edge has length zero.

Proof Suppose $T$ has $l$ leaves. Then it has $n+l$ total edges and using the lengths of the edges as coordinates we can think of $w(T)$ as a subset of $\mathbb{R}^{n+l}$. For each leaf $l_{i}$ of $T$ there is a condition on the lengths of the edges in a weighting that translates into an affine hyperplane $H_{i}$ in $\mathbb{R}^{n+l}$. Then $w(T)$ is the intersection of all these hyperplanes with $[0,1]^{n+l}$.

Now these hyperplanes all pass through the point that corresponds to the root edge having length 1 and all other edges length zero. Therefore their intersection is another affine subspace of $\mathbb{R}^{n+l}$. To see that they intersect transversely, we check that each $H_{i}$ does not contain the intersection of the $H_{j}$ for $j \neq i$. Consider the point $p_{i}$ in $\mathbb{R}^{n+l}$ that assigns length 1 to each leaf edge except that corresponding to leaf $l_{i}$, and length 0 to all other edges (including the leaf edge for $l_{i}$ ). Since the equation for the hyperplane $H_{j}$ contains the length of exactly one leaf edge, this point $p_{i}$ is in

$$
\bigcap_{j \neq i} H_{j}
$$

but not in $H_{i}$. This shows that the $H_{i}$ do indeed intersect transversely. Therefore their intersection is an $n$-dimensional affine subspace $V$ of $\mathbb{R}^{n+l}$.

Finally, notice that, as long as $n>0, V$ passes through an interior point of $[0,1]^{n+l}$, for example, the point where all edges except the leaf edges have length $\varepsilon$ for some small $\varepsilon>0$ and the leaf edges then have whatever lengths they must have to obtain a weighting. It then follows that $w(T)=V \cap[0,1]^{n+l}$ is homeomorphic to $D^{n}$. If $n=0$, there is only one tree and its space of weightings is a single point, that is, $D^{0}$.

For the second statement, notice that the boundary of $w(T)$ is the intersection of $V$ with the boundary of the cube $[0,1]^{n+l}$. If a weighting includes an edge of length zero, it lies in this boundary. Conversely, a weighting in this boundary must have some edge with length either 0 or 1 . If the root edge has length 1 , all other edges must have length 0 . If some other edge has length 1 , the root edge must have length 0 . In any case, some edge has length 0 . 
Definition 3.10 For each finite set $A$, the assignment $T \mapsto w(T)$ determines a functor

$$
w(-): \mathrm{T}(A) \rightarrow \mathcal{U}
$$

where $\mathcal{U}$ is the category of unbased spaces. To see this we must define maps

$$
w(T / e) \rightarrow w(T)
$$

whenever $e$ is an internal edge in the $A$-labelled tree $T$. Given a weighting on $T / e$ we define a weighting on $T$ by giving edges in $T$ their lengths in $T / e$ with the edge $e$ having length zero. This is an embedding of $w(T / e)$ as a 'face' of the 'simplex' $w(T)$. It's easy to check that this defines a functor as claimed.

Let $w_{0}(T)$ be the subspace of $w(T)$ containing weightings for which either the root edge or some leaf edge has length zero. We set

$$
\bar{w}(T):=w(T) / w_{0}(T) .
$$

This is a based space with basepoint given by the point to which $w_{0}(T)$ has been identified. If $T$ is the tree with only one edge then $w_{0}(T)$ is empty. We use the convention that taking the quotient by the empty set is equivalent to adjoining a disjoint basepoint. So in this case, $\bar{w}(T)=S^{0}$.

The maps $w(T / e) \rightarrow w(T)$ clearly map $w_{0}(T / e)$ to $w_{0}(T)$ and so give us maps

$$
\bar{w}(T / e) \rightarrow \bar{w}(T) .
$$

For each finite set $A$, these form a functor

$$
\bar{w}(-): \mathrm{T}(A) \rightarrow \mathcal{T}
$$

where $\mathcal{T}$ is the category of based spaces.

Example 3.11 Figure 4 displays the spaces $w(T)$ for $T \in \mathrm{T}(3)$ and how the functor $w(-)$ fits them together. Recall that the poset $\mathrm{T}(3)$ has four objects: one minimal object (the tree with one vertex and three incoming edges) and three maximal objects (three binary trees with two vertices). As the picture shows, the functor $w(-)$ embeds a 1-simplex for the minimal object as one of the 1-dimensional faces of a 2 -simplex for each of the maximal objects. The subspaces $w_{0}(T)$ are outlined in bold. Collapsing these we get the functor $\bar{w}(-)$ which embeds $S^{1}$ (for the minimal object) as the boundary of $D^{2}$ (for each maximal object). 

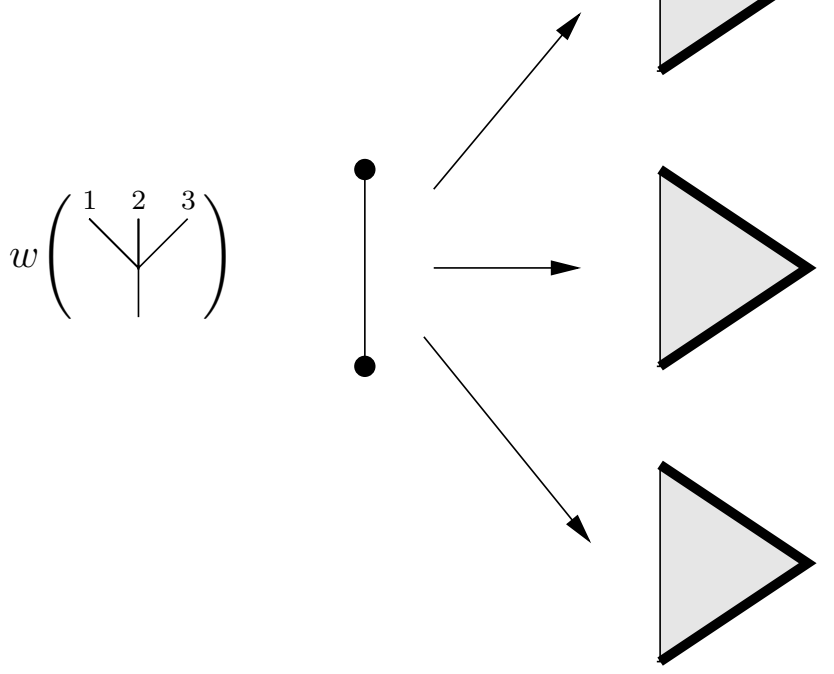
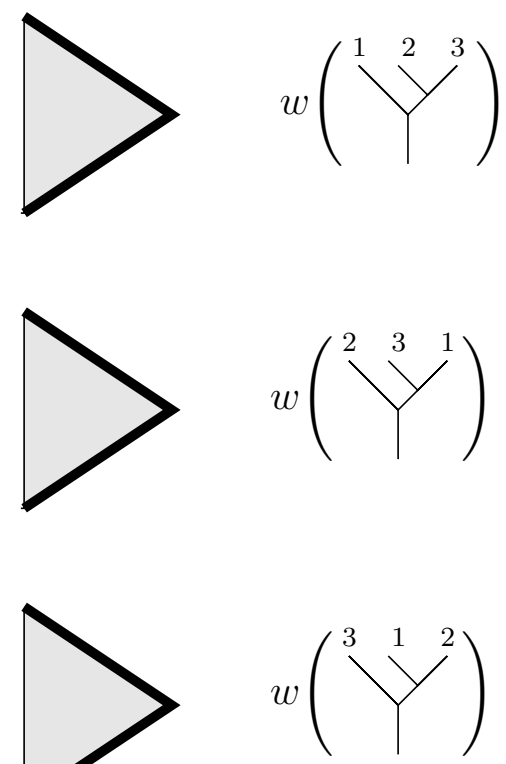

Figure 4: Spaces of weightings of trees with three leaves

\section{Bar constructions for reduced operads}

This section forms the heart of the paper. We show that by giving an explicit description of the simplicial bar construction in terms of trees, we can construct a cooperad structure on it. In Section 4.1 we give our definition of the bar construction $B(P)$ for an operad $P$ in $\mathcal{C}$. In Section 4.2 we show that this is isomorphic to the standard simplicial reduced bar construction on $P$. Then in Section 4.3 we prove the main result of this paper: that $B(P)$ admits a natural cooperad structure.

We will work in a fixed symmetric monoidal $\mathcal{T}$-category $(\mathcal{C}, \bar{\wedge}, S)$ where $\mathcal{T}$ is the category of based compactly-generated spaces and basepoint preserving maps. Since $\mathcal{T}$ is pointed, Proposition 1.13 implies that $\mathcal{C}$ too is pointed. We denote the null object in $\mathcal{C}$ also by $*$. We assume that $\mathcal{C}$ has all limits and colimits. The examples to bear in mind are $\mathcal{C}=\mathcal{T}$ itself and $\mathcal{C}=\mathcal{S} p$, which we take to be the category of $S$-modules of EKMM [6], although other categories of spectra could be used. We will use the notation developed in Section 1 for the enrichment, tensoring and cotensoring of $\mathcal{C}$ over $\mathcal{T}$.

Before we start we should stress that the constructions in this paper only apply 
to reduced operads and cooperads. That is, those for which the unit (or counit) map is an isomorphism. This is reflected in several places, most notably in the fact that our trees are not allowed to have vertices with only one incoming edge (see Remark [3.2). It is a necessary condition for our construction of the cooperad structure on $B(P)$.

\subsection{Definition of the bar construction}

We give two definitions of the bar construction for an operad. The first is somewhat informal and relies on $\mathcal{C}$ being the category of based spaces, but captures how we really think about these objects. The second is a precise formal definition as a coend in the category $\mathcal{C}$.

Definition 4.1 Let $P$ be a reduced operad in $\mathcal{T}$. The bar construction on $P$ is the symmetric sequence $B(P)$ defined as follows. A general point $p$ in $B(P)(A)$ consists of

- an isomorphism class of $A$-labelled trees: $T \in \mathrm{T}(A)$,

- a weighting on $T$ and,

- for each (internal) vertex $v$ of $T$, a point $p_{v}$ in the based space $P(i(v))$ (recall that $i(v)$ is the set of incoming edges of the vertex $v$ ),

subject to the following identifications:

- If $p_{v}$ is the basepoint in $P(i(v))$ for any $v$ then $p$ is identified with the basepoint $* \in B(P)(A)$.

- If the internal edge $e$ has length zero, we identify $p$ with the point $q$ given by

- the tree $T / e$,

- the weighting on $T / e$ in which an edge has the same length as the corresponding edge of $T$ in the weighting that makes up $p$

- $q_{u \circ v}$ given by the image under the composition map

$$
P(i(u)) \wedge P(i(v)) \rightarrow P(i(u \circ v))
$$

of $\left(p_{u}, p_{v}\right)$ (notice that $\left.i(u \circ v)=i(u) \circ_{v} i(v)\right)$,

\footnotetext{
${ }^{7}$ This is the inverse image under the injective map

$$
w(T / e) \rightarrow w(T)
$$

of the weighting corresponding to $p$. The condition that $e$ has length zero says precisely that the weighting for $p$ is in the image of this map.
} 
$-q_{t}=p_{t}$ for the other vertices $t$ of $T / e$.

- If a root or leaf edge has length zero, $p$ is identified with $* \in B(P)(A)$.

A bijection $\sigma: A \rightarrow A^{\prime}$ gives us an isomorphism $\sigma_{*}: B(P)(A) \rightarrow B(P)\left(A^{\prime}\right)$ by relabelling the leaves of the underlying trees. In this way, $B(P)$ becomes a symmetric sequence in $\mathcal{T}$.

Example 4.2 Consider $B(P)(1)$. There is only one tree with a single leaf and only one weighting on it. It has no vertices so $B(P)(1)$ does not depend at all on $P$. With the basepoint (which is disjoint in this case because nothing is identified to it) we get $B(P)(1)=S^{0}$.

Next consider $B(P)(2)$. Again there is only one tree, but this time it has a vertex (with two incoming edges) and the space of ways to weight the tree is the 1 -simplex $\Delta^{1}$. Making all the identifications we see that

$$
B(P)(2)=\Sigma P(2),
$$

the reduced suspension of $P(2)$.

Definition 4.3 (The functors $P_{A}$ ) A key ingredient of the general definition of the bar construction is that an operad $P$ in $\mathcal{C}$ determines a functor

$$
P_{A}(-): \mathrm{T}(A)^{\mathrm{op}} \rightarrow \mathcal{C} .
$$

where $\mathrm{T}(A)$, as always, is the poset of isomorphism classes of $A$-labelled trees ordered by edge collapse. For a tree $T$ we define

$$
P_{A}(T):=\bar{\bigwedge}_{\text {vertices } v \text { in } T} P(i(v))
$$

where we recall that $i(v)$ is the set of incoming edges to the vertex $v$. If $e$ is an internal edge in $T$ with endpoints $u$ and $v$ then there is a partial composition map

$$
P(i(u)) \bar{\wedge} P(i(v)) \rightarrow P(i(u \circ v)) .
$$

Using this we get a map

$$
P_{A}(T) \rightarrow P_{A}(T / e) .
$$

The associativity axioms for the operad $P$ ensure that these maps make $P_{A}(-)$ into a functor as claimed.

Recall from Definition 3.10 that we have a functor

$$
\bar{w}(-): \mathrm{T}(A) \rightarrow \mathcal{T}
$$

given by taking the space of weightings on a tree, modulo those for which a root or leaf edge has length zero. 
Definition 4.4 (Formal definition of the bar construction) Let the bar construction of the reduced operad $P$ be the symmetric sequence $B(P)$ defined by

$$
B(P)(A):=\bar{w}(-) \otimes_{\mathrm{T}(A)} P_{A}(-)=\int^{T \in \mathrm{T}(A)} \bar{w}(T) \otimes P_{A}(T) .
$$

This is the coend in $\mathcal{C}$ of the bifunctor

$$
\bar{w}(-) \otimes P_{A}(-): \mathrm{T}(A) \times \mathrm{T}(A)^{\mathrm{op}} \rightarrow \mathcal{C} .
$$

(See [15] for the theory of coends.) The definition of the coend is a colimit over a category whose objects are morphisms in $\mathrm{T}(A)$ and we will write the coend above as

$$
\underset{T \leq T^{\prime} \in \mathrm{T}(A)}{\operatorname{colim}} \bar{w}(T) \otimes P_{A}\left(T^{\prime}\right)
$$

when we need to manipulate it as such.

A bijection $A \rightarrow A^{\prime}$ induces an isomorphism of categories $\mathrm{T}(A) \rightarrow \mathrm{T}\left(A^{\prime}\right)$ by the relabelling of trees. If $T \mapsto T^{\prime}$ under this isomorphism then $P_{A}(T)=P_{A}\left(T^{\prime}\right)$ and $\bar{w}(T)=\bar{w}\left(T^{\prime}\right)$. Therefore we get an induced isomorphism $B(P)(A) \rightarrow$ $B(P)\left(A^{\prime}\right)$. This makes $B(P)$ into a symmetric sequence in $\mathcal{C}$.

Remark 4.5 To see that our two definitions of the bar construction are equivalent when $\mathcal{C}=\mathcal{T}$, recall that the coend is a quotient of the coproduct

$$
\bigvee_{T \in \mathrm{T}(A)} \bar{w}(T) \otimes P_{A}(T)
$$

That is, a point consists of a weighted tree together with elements of the $P(i(v))$ for vertices $v$ subject to some identifications. The maps $P_{A}(T) \rightarrow P_{A}(T / e)$ and $\bar{w}(T / e) \rightarrow \bar{w}(T)$ encode the identifications made in Definition 4.1

Remark 4.6 Our definition of the bar construction is rather reminiscent of the geometric realization of simplicial sets or spaces. This line of thought leads to the definition of an arboreal object in $\mathcal{C}$ as a functor

$$
\mathrm{T}(A)^{\mathrm{op}} \rightarrow \mathcal{C}
$$

in which $\mathrm{T}(A)$ plays the role of the simplicial indexing category $\Delta$ for simplicial sets. With the spaces of weightings $\bar{w}(T)$ playing the role of the topological simplices, the bar construction $B(P)$ can be thought of as the geometric realization of the arboreal object $P_{A}(-)$. We will formalize and extend these ideas in future work [4]. 
Remark 4.7 The $W$-construction of Boardman and Vogt (also sometimes called the bar construction) is defined in a very similar manner to $B(P)$. It uses slightly different spaces of trees and produces an operad instead of a cooperad. See [19] for details. Benoit Fresse has noticed a relationship between $W(P)$ and $B(P)$, namely that

$$
B(P)=\Sigma \operatorname{Indec}(W(P))
$$

where $\Sigma$ is a single suspension (that is, tensoring with $S^{1}$ ) and Indec denotes the 'operadic indecomposables functor'. It is the cooperad structure on $\Sigma$ Indec $(W(P))$, corresponding to that on $B(P)$, that was described by Salvatore in [17.

Example 4.8 Let $\mathcal{A} s s$ be the operad for associative monoids in unbased spaces. This is given by

$$
\mathcal{A s s}(n):=\Sigma_{n}
$$

(with the discrete topology and regular $\Sigma_{n}$-action). The composition maps are the inclusions given by identifying

$$
\Sigma_{r} \times \Sigma_{n_{1}} \times \cdots \times \Sigma_{n_{r}}
$$

with a subgroup of $\Sigma_{n_{1}+\cdots+n_{r}}$. We obtain an operad $\mathcal{A} s s_{+}$in $\mathcal{T}$ by adding a disjoint basepoint to each of the terms of $\mathcal{A} s s$. Let us calculate $B\left(\mathcal{A} s s_{+}\right)$.

The points $p_{v} \in \mathcal{A} s s_{+}(i(v))$ required by Definition 4.1 can be thought of as determining an order on the incoming edges to vertices of a tree. This allows us to identify a point in $B\left(\mathcal{A} s s_{+}\right)(n)$ with a planar weighted tree with leaves labelled $1, \ldots, n$. This breaks $B\left(\mathcal{A} s s_{+}\right)(n)$ up into a wedge of $n$ ! terms, each corresponding to an ordering of the leaves of the trees involved.

As we now show, each of these terms is an $(n-1)$-sphere. Think of constructing a planar weighted tree with leaves labelled in a fixed order (say, $1, \ldots, n$ ) by the following method. Connect the first leaf to the root with an edge of length 1. Then attach the second leaf at some point along the edge already drawn. Attach the third leaf at some point along the path from the second leaf to the root, and so on. The space of choices made in doing all this is $[0,1]^{n-1}$ and we obtain precisely the planar weighted trees we want in this manner (see Figure 5). The root edge or a leaf edge will have length zero if and only if at least one of our choices was either 0 or 1 . Hence the space we want is obtained by identifying the boundary of $[0,1]^{n-1}$ to a basepoint. This gives $S^{n-1}$.

Therefore we have

$$
B\left(\mathcal{A} s s_{+}\right)(n) \cong S^{n-1} \wedge\left(\Sigma_{n}\right)_{+}
$$



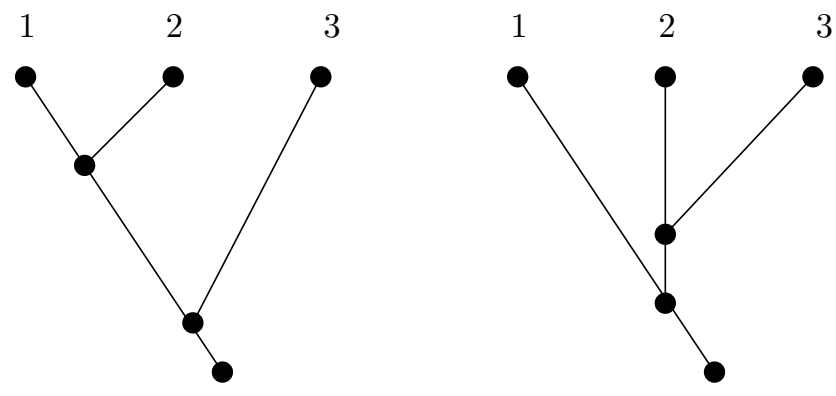

Figure 5: Constructing planar weighted trees

where $\Sigma_{n}$ acts trivially on the $S^{n-1}$ term and by translation on the nonbasepoints of $\left(\Sigma_{n}\right)_{+}$.

We can also picture what happens for $n=3$ in terms of sticking together the spaces $\bar{w}(T) \wedge \mathcal{A} s s_{3}(T)_{+}$for $T \in \mathrm{T}(3)$. The $\bar{w}(T)$ are the quotients of the spaces pictured in Figure 4 by the subspaces outlined in bold. To make up $B\left(\mathcal{A} s s_{+}\right)(3)$ we need six copies of the 1 -simplex (corresponding to the points in $\mathcal{A} s s(3)$ ) and twelve copies of the 2-simplex. (There are four points in $\mathcal{A} s s(2) \times \mathcal{A} s s(2)$ and three trees of this type.) These fit together to form six disjoint copies of the space of Figure 6, one for each permutation of 1,2,3. The type of tree used to form each part is shown. When we collapse the bold subspaces to the basepoint

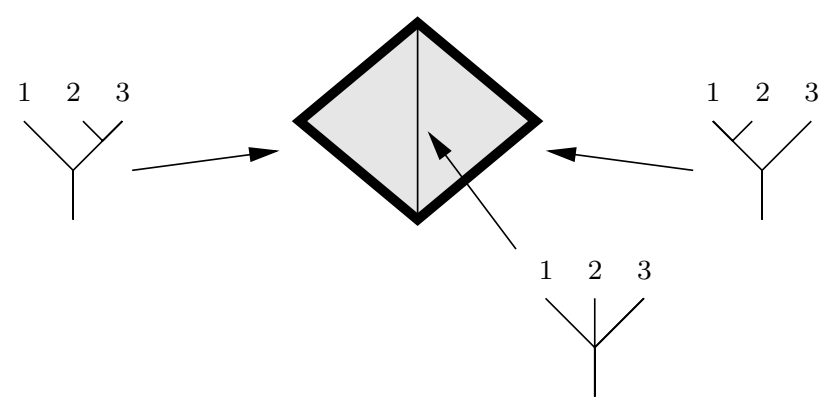

Figure 6: One sixth of $B\left(\mathcal{A s s} s_{+}\right)(3)$

we get a wedge of six copies of $S^{2}$ as expected. 


\subsection{Relation to the simplicial bar construction}

In this section we show that $B(P)$ is isomorphic to the geometric realization of the standard simplicial bar construction on the reduced operad $P$. This simplicial bar construction can be defined for any augmented monoid in a monoidal category 8 We have seen (Proposition 2.9) that under the right conditions an operad is just a monoid for the monoidal product on the category of symmetric sequences given by the composition product $\circ$. To define the simplicial bar construction in general (that is, without the assumption that $\bar{\wedge}$ commutes with finite coproducts) we must say what we mean by higher iterates of $\circ$. For this we use the following natural extension of the three-way product introduced in the proof of Lemma 2.8

Definition 4.9 (Iterated composition product) The composition product of the symmetric sequences $M_{1}, \ldots, M_{r}$ is the symmetric sequence given by

$$
\left(M_{1} \circ \cdots \circ M_{r}\right)(A):=\bigvee_{A_{i}=\amalg_{a \in A_{i-1}} A_{i, a}} M_{1}\left(A_{1}\right) \bar{\wedge} \bigwedge_{a \in A_{1}} M_{2}\left(A_{2, a}\right) \bar{\wedge} \cdots \bar{\wedge} \bigwedge_{a \in A_{r-1}} M_{r}\left(A_{r, a}\right)
$$

for each finite set $A=A_{r}$. Here we are taking the coproduct over partitions of $A$ into subsets $A_{r, a}$ indexed over $a \in A_{r-1}$, partitions of $A_{r-1}$ indexed over $A_{r-2}$, and so on. Equivalently we can view this coproduct as indexed over sequences of $r-1$ partitions of $A$, each a refinement of the next.

Remark 4.10 There is a natural map from $\left(M_{1} \circ \cdots \circ M_{r}\right)$ to any of the symmetric sequences obtained by choosing ways to bracket this expression. All the 'obvious' diagrams relating these maps commute. If $\bar{\wedge}$ commutes with finite coproducts in $\mathcal{C}$ then all these maps are isomorphisms and reflect the associativity isomorphisms of the monoidal product $\circ$.

Definition 4.11 (Simplicial bar construction) Let $P$ be a reduced operad in $\mathcal{C}$. The simplicial bar construction $\mathcal{B}_{\bullet}(P)$ is the simplicial object in the category of symmetric sequences on $\mathcal{C}$ with

$$
\mathcal{B}_{k}(P)=\underbrace{P \circ \cdots \circ P}_{k} .
$$

For $i=1, \ldots, k-1$, face maps

$$
d_{i}: \underbrace{P \circ \cdots \circ P}_{k} \rightarrow \underbrace{P \circ \cdots \circ P}_{k-1}
$$

\footnotetext{
${ }^{8}$ See [16, Section II.2.3] for a discussion of different forms of the simplicial bar construction.
} 
are given by

$$
\cdots \circ P \circ P \circ \cdots \rightarrow \cdots \circ(P \circ P) \circ \cdots \rightarrow \cdots \circ P \circ \cdots
$$

where we are using the operad composition $P \circ P \rightarrow P$ to compose the $i^{\text {th }}$ and $i+1^{\text {th }}$ factors. The maps $d_{0}$ and $d_{k}$ are given by applying the augmentation map $P \rightarrow I$ to the first and last copies of $P$ respectively. Degeneracy maps

$$
s_{j}: \underbrace{P \circ \cdots \circ P}_{k} \rightarrow \underbrace{P \circ \cdots \circ P}_{k+1}
$$

are given for $j=0, \ldots, k$ by using the unit map $I \rightarrow P$ to insert a copy of $P$ between the $j^{\text {th }}$ and $(j+1)^{\text {th }}$ factors:

$$
\cdots \circ P \circ P \circ \cdots \cong \cdots \circ P \circ I \circ P \circ \cdots \rightarrow \cdots \circ P \circ P \circ P \circ \cdots .
$$

Remark 4.12 It is sufficient for this definition that $P$ be augmented. However, we need $P$ to be reduced to make the following identification of the simplicial bar construction with $B(P)$ as defined previously.

Proposition 4.13 Let $P$ be a reduced operad in $\mathcal{C}$. Then the geometric realization 19 of $\mathcal{B}_{\bullet}(P)$ is isomorphic to the bar construction $B(P)$.

Proof We give the proof for $\mathcal{C}=\mathcal{T}$ (which is the only case we require in this paper) based on the informal description of $B(P)$ in Definition 4.1 The same idea could be used to write a proof that works for any $\mathcal{C}$ using the formal definition of $B(P)$ as a coend.

The idea is that the iterated composition products that make up the simplicial bar construction can be thought of in terms of sequences of partitions which in turn are related to trees of the type we are using to define $B(P)$.

We first give an explicit description of the $n$-simplices in $\mathcal{B}_{\bullet}(P)(A)$. These are given by the object

$$
\underbrace{P \circ \cdots \circ P}_{n}(A) \text {. }
$$

Enlarging on the last sentence of Definition 4.9 we can write this as a coproduct over all sequences of partitions

$$
\widehat{0}=\lambda_{0} \leq \lambda_{1} \leq \cdots \leq \lambda_{n-1} \leq \lambda_{n}=\widehat{1}
$$

\footnotetext{
${ }^{9}$ The geometric realization of a simplicial symmetric sequence is defined pointwise: $|X|(A)=|X(A)|$. Note that a simplicial symmetric sequence is the same thing as a symmetric sequence of simplicial objects.
} 
of the set $A$, where $\lambda \leq \mu$ if $\lambda$ is finer than $\mu$ (if two elements of $A$ are in the same block in $\lambda$, they are also in the same block in $\mu$ ) and $\widehat{0}, \widehat{1}$ are the minimal and maximal partitions with respect to this order. The terms in the coproduct are appropriate smash products of the $P(r)$. We get a factor of $P(r)$ every time one of the blocks of one of the partitions breaks up into $r$ blocks in the next partition along.

A point in the geometric realization $\left|\mathcal{B}_{\bullet}(P)\right|$ can be represented by a point in the topological $n$-simplex $\Delta^{n}$ together with a choice of sequence of partitions as described above and a point in the appropriate smash product of the spaces $P(r)$.

A sequence of partitions determines an $A$-labelled tree $T$ as follows. (See Figure 7 for an example when $n=3$.) Take a vertex for each block of each $\lambda_{i}$ for $i=1, \ldots, n$. Add a root, and a leaf for each element of $A$. Two vertices are joined by an edge if they come from consecutive partitions of the sequence and the block for one is contained in the block for the other. Finally we add a root edge from the $\lambda_{n}$ vertex to the root and a leaf edge from each leaf to the corresponding $\lambda_{1}$ vertex. (Notice that vertices in this tree might have only one input edge - let's allow this for the moment.)

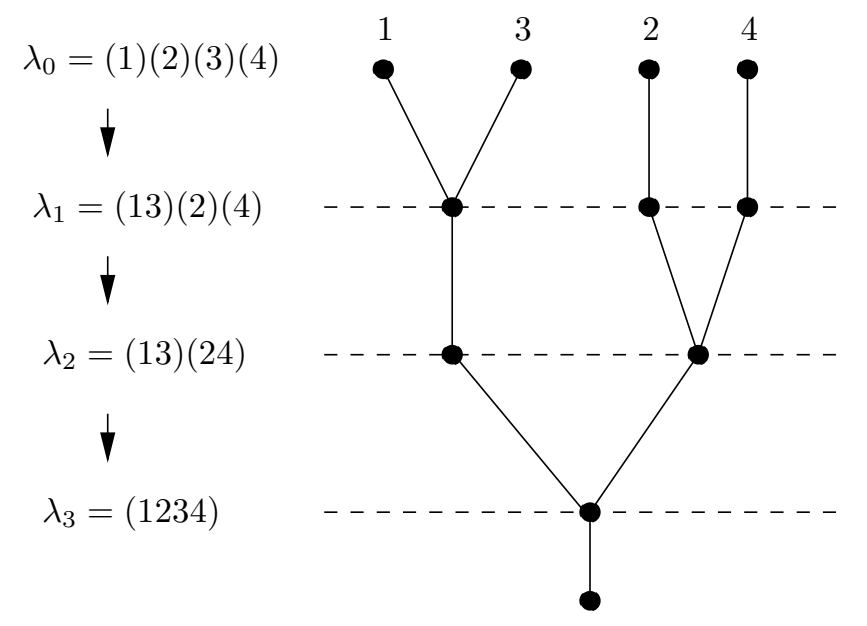

Figure 7: Producing trees from sequences of partitions

A point in $\Delta^{n}$ determines a weighting on the tree we have just constructed. Thinking of $\Delta^{n}$ as the subspace of $\mathbb{R}^{n+1}$ with $x_{0}+\cdots+x_{n}=1$ and $x_{i} \geq 0$, we get a weighting by giving the root edge length $x_{0}$, the edges connecting the vertices for $\lambda_{i-1}$ to the vertices for $\lambda_{i}$ length $x_{i}$ and the leaf edges length $x_{n}$. 
We can now remove the vertices with only one input edge, connecting their input and output edges. This gives us a point in $w(T)$ for some tree $T$ in the sense of Definition 3.1 .

Finally notice that because $P(1)=S^{0}$ (as $P$ is reduced), the smash product of spaces $P(r)$ determined by the sequence of partitions is precisely $P_{A}(T)$. Therefore we actually obtain a point in $B(P)(A)$.

It remains to show that this process sets up a homeomorphism between the geometric realization $\left|\mathcal{B}_{\bullet}(P)(A)\right|$ and $B(P)(A)$. There are a couple of key steps. Firstly the degeneracy maps in the simplicial bar construction are isomorphisms on terms in the coproduct. These correspond to inserting lots of vertices with one input edge in our trees, which are then removed by our construction. So we only have to worry about the identifications made by the face maps. The face maps are given by removing partitions from the sequences, which corresponds to edge collapse. Hence the identifications made in defining $B(P)$ are the same as those in defining the realization of $\mathcal{B}_{\bullet}(P)$. This completes the proof.

\subsection{Cooperad structure on the bar construction}

Up to this point, all we have done is identify the simplicial bar construction on a reduced operad in terms of trees. The main point of this paper is that this identification allows us to see that there is a cooperad structure on the bar construction. In this section we describe that structure. The key to getting the cooperad cocomposition maps is the process of grafting (or rather ungrafting) trees.

Definition 4.14 (Tree grafting) Let $T$ be an $A$-labelled tree, let $U$ be a $B$-labelled tree and let $a$ be an element of $A$. We define the grafting of $U$ onto $T$ at $a$ to be the tree $T \cup_{a} U$ obtained by identifying the root edge of $U$ to the leaf edge of $T$ corresponding to $a$. Figure 8 below illustrates this process.

We denote the newly identified edge by $e_{a}$. Every other edge of $T \cup_{a} U$ comes either from $T$ or from $U$. The vertices of $T \cup_{a} U$ are the vertices of $T$ together with the vertices of $U$ (and they have the same number of incoming edges). Finally there is a natural $A \cup_{a} B$-labelling of $T \cup_{a} U$, given by combining the labellings of $T$ and of $U$.

We say that an $A \cup_{a} B$-labelled tree is of type $(A, B)$ if it is of the form $T \cup_{a} U$ for an $A$-labelled tree $T$ and a $B$-labelled tree $U$. The next lemma says that an $A \cup_{a} B$-labelled tree is a grafting in at most one way. This is trivial but crucial to the construction of the cooperad structure maps below. 


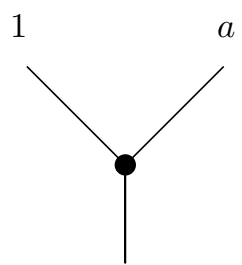

$T$
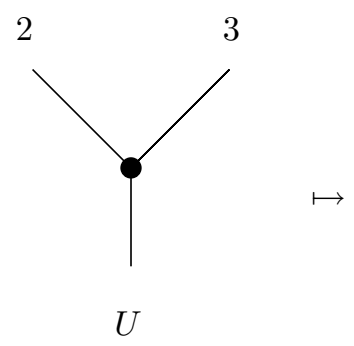

$U$

Figure 8: Tree grafting

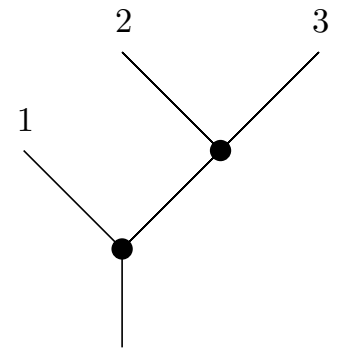

$$
T \cup_{a} U
$$

Lemma 4.15 For any $A \cup_{a} B$-labelled tree $V$ there is at most one pair $(T, U)$ such that $V=T \cup_{a} U$.

Proof In the grafted tree $T \cup_{a} U$ the 'upper' endpoint of the edge $e_{a}$ is a vertex whose 'parent leaves' are labelled precisely by the elements of $B$. There can be at most one such vertex $v$ in $V$ and cutting along the edge immediately below $v$ produces the trees $T, U$ that make up $V$.

Definition 4.16 To give $B(P)$ a cooperad structure we have to define maps

$$
B(P)\left(A \cup_{a} B\right) \rightarrow B(P)(A) \pi B(P)(B)
$$

for finite sets $A, B$ and $a \in A$. A point $p$ in $B(P)\left(A \cup_{a} B\right)$ consists of a weighted tree $V$ labelled by $A \cup_{a} B$ together with elements of $p_{v} \in P(i(v))$ for vertices $v$ of $V$. We treat two cases:

(1) If $V$ is not of the form $T \cup_{a} U$ for an $A$-labelled tree $T$ and a $B$-labelled tree $U$, then we will map $p$ to the basepoint on the right-hand side of (4.17).

(2) If $V$ is of this form (that is, it is of type $(A, B)$ ) then things are more interesting. Below we describe how the map (4.17) is defined in this case.

Since $V$ is of type $(A, B)$, Lemma4.15tells us that there is a unique $A$-labelled tree $T$ and a unique $B$-labelled tree $U$ such that $V=T \cup_{a} U$. We use these trees as the basis for elements $q \in B(P)(A)$ and $r \in B(P)(B)$ respectively. What remains to be seen is how the weighting and vertex labels of $V$ determine weightings and vertex labels for $T$ and $U$. 
The vertex labels are easy because the vertices of $T \cup_{a} U$ consist of the vertices of each of $T$ and $U$ with the same numbers of input edges. Therefore we take

$$
q_{v}:=p_{v} \in P(i(v))
$$

for vertices $v$ of $T$ and

$$
r_{u}:=p_{u} \in P(i(v))
$$

for vertices $u$ of $U$.

The way in which a weighting on $T \cup_{a} U$ determines weightings on $T$ and $U$ is the key part of our construction. This comes about via a map

$$
\bar{w}\left(T \cup_{a} U\right) \rightarrow \bar{w}(T) \wedge \bar{w}(U)
$$

(recall that $\bar{w}(-)$ is the space of weightings on a tree with those that have zero length root or leaf edges identified to a basepoint).

So take a weighting of $T \cup_{a} U$. Define a weighting on $T$ by giving the edges the same lengths they had in $T \cup_{a} U$ and giving the leaf edge for $a$ the necessary length to make the root-leaf distances equal to 110 Next define a weighting on $U$ by taking the lengths from $T \cup_{a} U$ and scaling up by a constant factor to make the root-leaf distances equal to 1 (the length of the root edge of $U$ comes from the length of the edge $e_{a}$ in $\left.T \cup_{a} U\right)$. The scaling factor is the inverse of the total length of the $U$ part of $T \cup_{a} U$. The only time this doesn't work is if all the $U$-edges in $T \cup_{a} U$ (including $e_{a}$ ) are of length zero. However in that case the weighting we just defined on $T$ has a leaf edge of length zero and so is the basepoint in $\bar{w}(T)$. This is almost enough to define a map of the form (4.18). The only thing left to check is that if a leaf or root edge of $T \cup_{a} U$ is of length zero then the same is true of either of the chosen weightings on $T$ and $U$. This is clear. Figure 9 illustrates a particular case of the map (4.18).

This completes the definition of the cooperad structure maps (4.17):

$$
B(P)\left(A \cup_{a} B\right) \rightarrow B(P)(A) \bar{\wedge} B(P)(B)
$$

given, in summary, by:

$$
p=\left(V,\left\{p_{v}\right\}\right) \mapsto \begin{cases}q=\left(T,\left\{p_{v}\right\}_{v \in T}\right), r=\left(U,\left\{p_{v}\right\}_{v \in U}\right) & \text { if } V=T \cup_{a} U ; \\ * & \text { otherwise. }\end{cases}
$$

with the weightings on $T, U$ given by the map (4.18) just constructed.

We still have to check that these maps are well-defined. To see this we have to look at the identifications made in the definition of $B(P)\left(A \cup_{a} B\right)$ :

\footnotetext{
${ }^{10}$ Intuitively, we have collapsed the $U$ part of the tree to a single edge with the same overall length.
} 

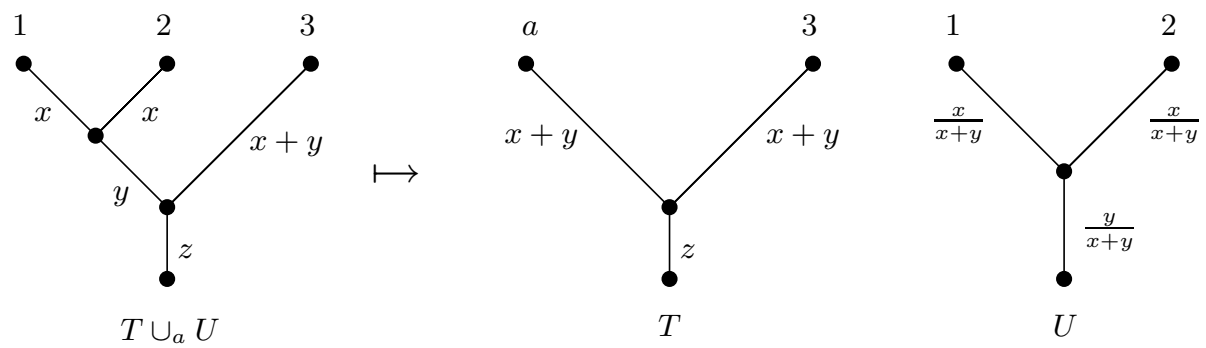

Figure 9: The map $\bar{w}\left(T \cup_{a} U\right) \rightarrow \bar{w}(T) \wedge \bar{w}(U)$

- If $p_{v}$ equals the basepoint in $P(i(v))$ for any vertex $v \in V$ then the same will be true of the corresponding vertex in either $T$ or $U$. Hence such a $p$ maps to the basepoint.

- If an interior edge $e$ of the tree $V$ underlying the point $p$ is of length zero, $p$ is identified with another point $p^{\prime}$ as described in Definition 4.1 We have various possibilities:

(1) $V$ is not of the form $T \cup_{a} U$ in which case neither is $V / e$ and both $p$ and $p^{\prime}$ map to the basepoint.

(2) $\quad V=T \cup_{a} U$ and $e$ corresponds to an internal edge of $T$. In this case, the points $q$ and $q^{\prime}$ will be identified via the collapse of that edge, and the points $r$ and $r^{\prime}$ will be equal. So $p$ and $p^{\prime}$ map to the same element of $B(P)(A) \bar{\wedge} B(P)(B)$.

(3) $V=T \cup_{a} U$ and $e$ corresponds to an internal edge of $U$. This is similar to case (2).

(4) $V=T \cup_{a} U$ and $e$ is the edge $e_{a}$ obtained from identifying the root edge of $U$ with the $a$-leaf edge of $T$. In this case $V / e$ is no longer of the form $T \cup_{a} U$ and so $p^{\prime}$ maps to the basepoint. But in the weighting on $U$ determined by that on $T \cup_{a} U$ the root edge has length scaled up from the length of $e_{a}$ which is therefore zero. So the point $r$ is the basepoint in $B(P)(B)$ and so $p$ also maps to the basepoint.

- We have already checked in the definition of the map (4.18) that if a root or leaf edge in $p$ is of length zero, then the same is true of at least one of $q$ and $r$. Therefore such a $p$ maps to the basepoint in $B(P)(A) \wedge B(P)(B)$.

This completes the check that our maps (4.17) are well-defined. The final piece of the cooperad structure for $B(P)$ is a counit map $B(P)(1) \rightarrow S^{0}$. But we 
already saw that $B(P)(1) \cong S^{0}$ (in the based space case) so our counit is this isomorphism. Note that this means $B(P)$ turns out to be a reduced cooperad.

Example 4.19 The map

$$
B(P)(\{1,2,3\}) \rightarrow B(P)(\{a, 3\}) \wedge B(P)(\{1,2\})
$$

is pictured in Figure 9. The left-hand side (with vertices labelled by elements of $P(2))$ represents a point $p$ of $B(P)(\{1,2,3\})$. The two trees on the righthand side (with vertices labelled by those same elements in the obvious way) represent the image of $p$ in $B(P)(\{a, 3\}) \bar{\wedge} B(P)(\{1,2\})$. In this example, all points that are based on trees of shapes other than that shown are mapped to the basepoint.

We will save for later the task of checking that these maps do indeed give us a cooperad structure. First we translate Definition 4.16] into the categorytheoretic language needed to define the cocomposition maps for a general $\mathcal{C}$. To do this, we notice that the 'ungrafting' process more-or-less makes our categories $\mathrm{T}(A)$ into a cooperad of categories. To make this precise, we describe an 'add a disjoint basepoint' functor for categories.

Definition 4.20 (Categories with initial objects) Write Cat + for the category in which an object is a (small) category $\mathrm{C}_{+}$together with an initial object $*$ such that $\operatorname{Hom}_{\mathrm{C}_{+}}(X, *)$ is empty for all $X \neq *$. The morphisms in Cat $\mathrm{C}_{+}$are functors that preserve the initial objects.

There is a functor from the category Cat of all (small) categories to Cat + given by adding an initial object with the correct morphisms to a category $\mathrm{C}$ to obtain $\mathrm{C}_{+}$. Note that every object in $\mathrm{Cat}_{+}$can be obtained in this way, but not every morphism in Cat + $_{+}$is given by adding an initial object to a morphism in Cat.

Define a symmetric monoidal product $\wedge$ on $\mathrm{Cat}_{+}$by

$$
C_{+} \wedge D_{+}:=C_{+} \times D_{+} / C_{+} \vee D_{+},
$$

where the wedge product is the disjoint union with the initial objects identified and the quotient identifies this wedge product to the initial object of the smash product. Notice that if $\mathrm{C}, \mathrm{D} \in$ Cat then

$$
C_{+} \wedge D_{+}=(C \times D)_{+} \cdot
$$

The unit for this product is the category $1_{+}$with two objects and a single morphism between them. 
In particular we write $\mathrm{T}(A)_{+}$for the category formed by adding an initial object to our poset of $A$-labelled trees $\mathrm{T}(A)$. The reason for making all these new definitions is then the following result.

Proposition 4.21 The categories $\mathrm{T}(A)_{+}$form a reduced cooperad in $\mathrm{Cat}_{+}$.

Proof The cocomposition maps have the form

$$
\mathrm{T}\left(A \cup_{a} B\right)_{+} \rightarrow \mathrm{T}(A)_{+} \wedge \mathrm{T}(B)_{+}=(\mathrm{T}(A) \times \mathrm{T}(B))_{+}
$$

and are given by 'ungrafting' trees. Take $V \in \mathrm{T}\left(A \cup_{a} B\right)$. If $V$ is a tree of type $(A, B)$ we map it to the pair $(T, U)$ where $T, U$ are the unique trees that graft together to give $V$ (see Lemma 4.15). If $V$ is not of type $(A, B)$ (or is the initial object) we map it to the initial object of the right-hand side.

First we must check that we have indeed given a functor here. Suppose that $V \leq V^{\prime}$ in $\mathrm{T}\left(A \cup_{A} B\right)$. The only interesting case is when $V$ is of type $(A, B)$, so maps to a pair $(T, U)$ on the right-hand side. We have to show two things: that $V^{\prime}$ is also of type $(A, B)$ with decomposition $\left(T^{\prime}, U^{\prime}\right)$ and then that $T \leq T^{\prime}$ and $U \leq U^{\prime}$. Well, let $e_{a}$ be the edge in $V$ at which the grafting took place. Since $V$ is obtained from $V^{\prime}$ by a sequence of edge collapses, $e_{a}$ must come from an edge $e_{a^{\prime}}$ in $V^{\prime}$ that is not collapsed in this sequence. This edge breaks $V^{\prime}$ into two parts and we can write $V^{\prime}=T^{\prime} \cup_{a^{\prime}} U^{\prime}$ for some trees $T^{\prime}, U^{\prime}$ with some labellings (a priori, not necessarily by $A$ and $B$ ). But it is now clear that $U^{\prime}$ must yield $U$ after undergoing some edge collapses. So $U^{\prime} \in \mathrm{T}(B)$ and $U \leq U^{\prime}$. Similarly, $T^{\prime} \in \mathrm{T}(A)$ and $T \leq T^{\prime}$ (after relabelling $a^{\prime}$ by $a$ ).

Notice that $\mathrm{T}(1)_{+}$is isomorphic to the unit $1_{+}$for the symmetric monoidal structure on $\mathrm{Cat}_{+}$. We take as unit map the (unique) isomorphism $1_{+} \rightarrow \mathrm{T}(1)_{+}$.

It still remains to check that the cooperad axioms do indeed hold for our cocomposition maps. This is simple and we leave it to the reader.

Remark 4.22 The original categories $\mathrm{T}(A)$ in fact already form an operad in Cat with composition maps given by grafting rather than ungrafting. This operad structure is effectively what is used by Boardman and Vogt to define their $W$-construction.

The next step is to show that the bar construction can be defined as a coend in $\mathrm{T}(A)_{+}$instead of $\mathrm{T}(A)$. 
Lemma 4.23 Let $P$ be a reduced operad in $\mathcal{C}$. The functors $\bar{w}(-)$ and $P_{A}(-)$ on $\mathrm{T}(A)$ naturally extend to functors

$$
\bar{w}(-): \mathrm{T}(A)_{+} \rightarrow \mathcal{T}
$$

and

$$
P_{A}(-):\left(\mathrm{T}(A)_{+}\right)^{o p} \rightarrow \mathcal{C}
$$

and we have

$$
B(P)(A)=\int^{T \in \mathrm{T}(A)_{+}} \bar{w}(T) \otimes P_{A}(T) .
$$

Proof We set $\bar{w}(*)=*_{\mathcal{T}}$ and $P_{A}(*)=*_{\mathcal{C}}$ with the necessary definition on morphisms (given by the fact that $*_{\mathcal{T}}$ is an initial object in $\mathcal{T}$ and $*_{\mathcal{C}}$ is a terminal object in $\mathcal{C})$. It is then clear that $* \in \mathrm{T}(A)_{+}$does not contribute anything to the coend which therefore reduces to the previous definition of $B(P)(A)$.

The maps 4.18) of Definition 4.16 are still the key ingredients in constructing the cooperad maps for $B(P)$.

Lemma 4.24 The maps

$$
\bar{w}\left(T \cup_{a} U\right) \rightarrow \bar{w}(T) \wedge \bar{w}(U)
$$

previously defined form part of a natural transformation

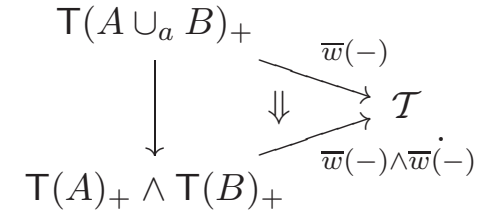

Proof The bottom functor here is defined in the obvious way on $\mathrm{T}(A) \times \mathrm{T}(B)$ and sends $*$ to $*$. For $V \in \mathrm{T}\left(A \cup_{a} B\right)$ not of type $(A, B)$, the corresponding part of the natural transformation is

$$
\bar{w}(V) \rightarrow * .
$$

The only really interesting naturality square comes from $V \leq V^{\prime}$ with $V^{\prime}$ of type $(A, B)$ and $V$ not. The square that must commute in this case is

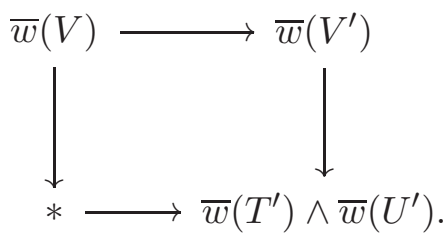


This is the content of part (4) of the checking we did towards the end of Definition 4.16 from any weighting on $V$, the weighting we get on $V^{\prime}$ will have length zero for the edge connecting the $T^{\prime}$-part to the $U^{\prime}$-part. Hence the root edge of the corresponding weighting on $U^{\prime}$ will have length zero. So we map into the basepoint of $\bar{w}\left(T^{\prime}\right) \wedge \bar{w}\left(U^{\prime}\right)$.

We have a corresponding result for the functors $P_{A}(-)$ of Definition 4.4.

Lemma 4.25 Let $P$ be a reduced operad in $\mathcal{C}$. Then there is a natural transformation

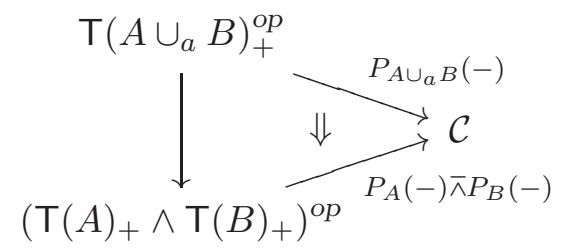

Proof In other words, given $V \in \mathrm{T}\left(A \cup_{a} B\right)$ we have maps

$$
P_{A \cup_{a} B}(V) \rightarrow P_{A}(T) \bar{\wedge} P_{B}(U)
$$

when $V=T \cup_{a} U$. There are obvious isomorphisms that we take for these maps. The naturality squares are easily seen to commute. Again the only one that seems like it might be interesting is for $V \leq V^{\prime}$ with $V^{\prime}$ of type $(A, B)$ and $V$ not. But in fact this square just turns out to be

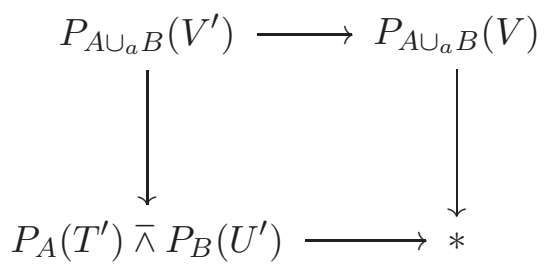

which is not so interesting after all.

Finally, we can give the formal construction of the cocomposition maps for the cooperad $B(P)$.

Definition 4.26 Let $P$ be a reduced operad in $\mathcal{C}$ and let $B(P)$ be the symmetric sequence of Definition 4.4. The cocomposition map

$$
B(P)\left(A \cup_{a} B\right) \rightarrow B(P)(A) \bar{\wedge} B(P)(B)
$$


is given by the following sequence of maps:

$$
\begin{aligned}
& B(P)\left(A \cup_{a} B\right)=\underset{V \leq V^{\prime} \in \mathrm{T}\left(A \cup_{a} B\right)_{+}}{\operatorname{colim}} \bar{w}(V) \otimes P_{A \cup_{a} B}\left(V^{\prime}\right)
\end{aligned}
$$

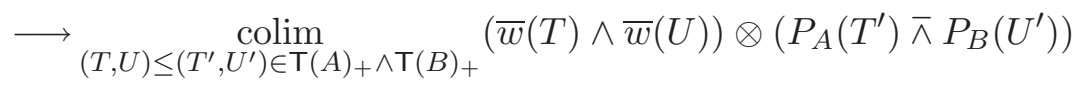

$$
\begin{aligned}
& \longrightarrow \operatorname{colim}_{(T, U) \leq\left(T^{\prime}, U^{\prime}\right) \in \mathbf{T}(A)_{+} \wedge \mathbf{T}(B)_{+}}\left(\bar{w}(T) \otimes P_{A}\left(T^{\prime}\right)\right) \bar{\wedge}\left(\bar{w}(U) \otimes P_{B}\left(U^{\prime}\right)\right) \\
& \longrightarrow\left(\operatorname{colim}_{T \leq T^{\prime} \in \mathbf{T}(A)_{+}} \bar{w}(T) \otimes P_{A}\left(T^{\prime}\right)\right) \bar{\wedge}\left(\operatorname{colim}_{U \leq U^{\prime} \in \mathbf{T}(B)_{+}} \bar{w}(U) \otimes P_{B}\left(U^{\prime}\right)\right) \\
& =B(P)(A) \bar{\wedge} B(P)(B) \text {. }
\end{aligned}
$$

The first map here comes from combining the natural transformations of Lemmas 4.24 and 4.25 The second is given by the transformation $d$ of Definition 1.10 It is for precisely this reason that the axiom giving us $d$ is necessary. The third map is given by universal properties of colimits. This completes the construction of the cooperad structure maps for $B(P)$.

The next task is to check that the maps we have described actually do make $B(P)$ into a cooperad. That is, we must check the duals of axioms (1)-(4) from Definition 2.2 The key step is to see that the maps (4.18) satisfy corresponding conditions.

Lemma 4.28 Let T, $U, V$ be $A-, B$ - and $C$-labelled trees respectively and let $a, a^{\prime} \in A$ and $b \in B$. Let $I$ denote the unique $*$-labelled tree. Recall that $\bar{w}(I)=S^{0}$. Then the following diagrams commute:

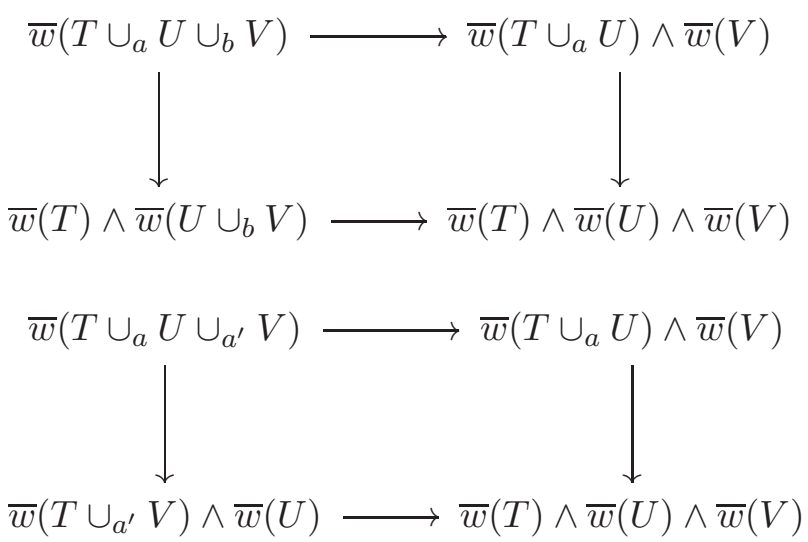




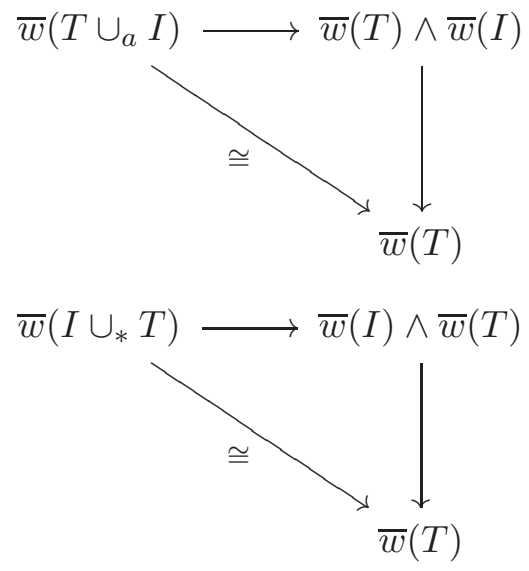

Proof The argument for diagram (1) is contained in Figure 10. A point in $\bar{w}\left(T \cup_{a} U \cup_{b} V\right)$ comes from a weighting of the grafted tree $T \cup_{a} U \cup_{b} V$. The top-left corner of Figure 10 shows a generic version of such a tree with some lengths labelled:

- $u$ is the length of the root edge.

- $v$ is the distance from the root vertex to the lower vertex of the edge that joins $U$ to $T$ (there may be intermediate vertices along this route, we let $v$ denote the total distance).

- $w$ is the length of the edge that joins $U$ to $T$.

- $x$ is the distance from the upper vertex of that edge to the lower vertex of the edge that joins $V$ to $U$.

- $y$ is the length of the edge that joins $V$ to $U$.

- $z$ is the remaining distance to any of the leaves of $V$.

Figure 10] shows that whichever way we map our weighted tree around diagram (1) we get the same result. (Note that if $y+z$ or $w+x+y+z$ equals to zero, then $z=0$ and we are the basepoint in every corner of diagram (1).) We therefore conclude that diagram (1) commutes.

Diagram (2) is similar to (1) but easier. For diagram (3), notice that the image in $\bar{w}(T)$ of a weighting of $T \cup_{a} I$ will be effectively the same weighting. The image in $\bar{w}(I)=S^{0}$ will be the non-basepoint unless the leaf edge for $a$ has length zero. But if this is the case our starting point was the basepoint in $\bar{w}\left(T \cup_{a} I\right)$. This shows that the diagram commutes.

For diagram (4), the image in $\bar{w}(T)$ of a weighting of $I \cup_{*} T$ will again be the very same weighting (no scaling up is necessary). The image in $\bar{w}(I)=S^{0}$ will always be the non-basepoint. Therefore this diagram also commutes. 


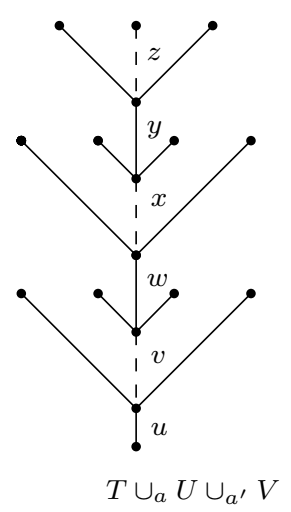

I
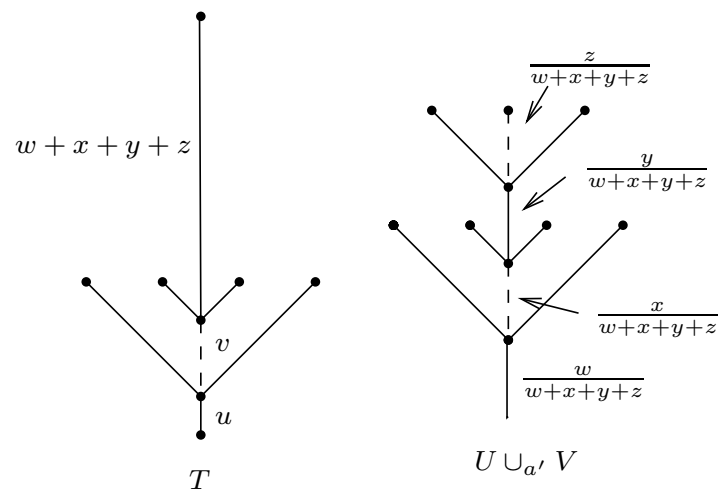

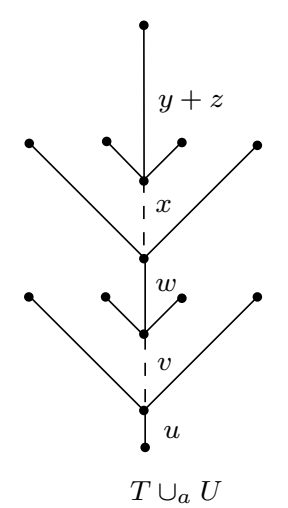

$\stackrel{\infty}{\infty}$
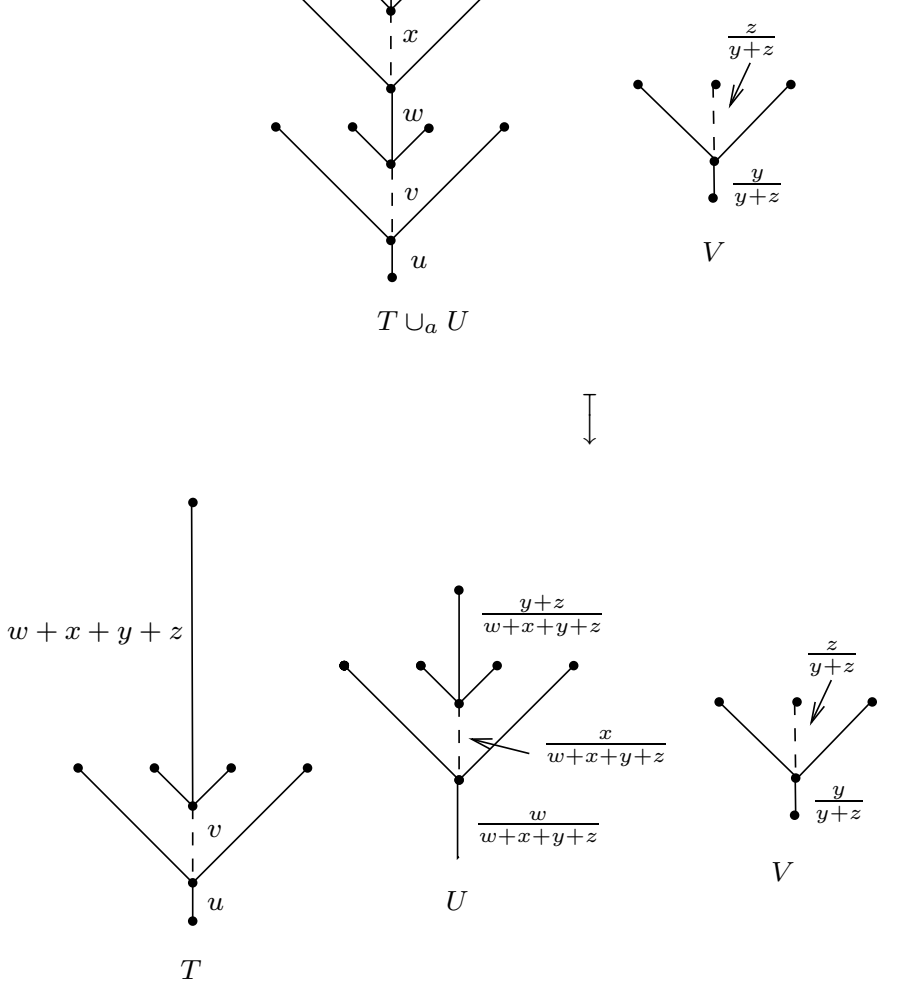

$T$

Figure 10: Commutativity of diagram (1) of Lemma 4.28 The dashed lines represent sequences of possibly more than one edge. 
We are now in a position to state the main result of this paper.

Theorem 4.29 Let $P$ be a reduced operad in the symmetric monoidal $\mathcal{T}$ category $\mathcal{C}$. The maps of Definition [4.16] make $B(P)$ into a reduced cooperad in $\mathcal{C}$.

Proof We give the formal argument for the maps of Definition 4.26. To fit the relevant diagrams onto a page we need some new notation. Let's write

$$
\bar{w}(T, U):=\bar{w}(T) \wedge \bar{w}(U)
$$

and

$$
P_{A, B}\left(T^{\prime}, U^{\prime}\right):=P_{A}\left(T^{\prime}\right) \bar{\wedge} P_{B}\left(U^{\prime}\right) .
$$

Figure [1] then shows the diagram that has to commute for the dual of axiom (1) of Definition 2.2 to hold for $B(P)$. The key to showing that this commutes is putting

$$
\operatorname{colim}_{\substack{T \leq T^{\prime} \in \mathbf{T}(A)_{+} \\ U \leq U^{\prime} \in \mathbf{T}(B)_{+} \\ V \leq V^{\prime} \in \mathbf{T}(C)_{+}}} \bar{w}(T, U, V) \otimes P_{A, B, C}\left(T^{\prime}, U^{\prime}, V^{\prime}\right)
$$

into the center of the square. We've connected this to the top and left sides of the square using maps similar to the first map in Definition 4.26. We've connected it to the right and bottom sides using maps of the form $d$ from Definition 1.10. It's then enough to show that the four smaller squares commute.

The top-left square commutes because of diagram (1) in Lemma 4.28 The bottom-left and top-right squares commute because of the naturality of the transformations $d$. The bottom-right square commutes because it is an example of the associativity axiom we required of our $d$ transformations in 1.10

This completes the verification of the dual of axiom (1) of Definition 2.2. For axiom (2) the argument is similar, but using diagram (2) of Lemma 4.28. For the duals of axioms (3) and (4) we use the unit axiom for the transformations $d$ together with diagrams (3) and (4) of Lemma 4.28. We leave the reader to fill in the details of these proofs.

\section{Cobar constructions for reduced cooperads}

We now dualize to cooperads. The cobar construction for a cooperad is strictly dual to the bar construction for an operad. More precisely, recall that a cooperad $Q$ in a category $\mathcal{C}$ is the same thing as an operad $Q^{\text {op }}$ in the opposite 


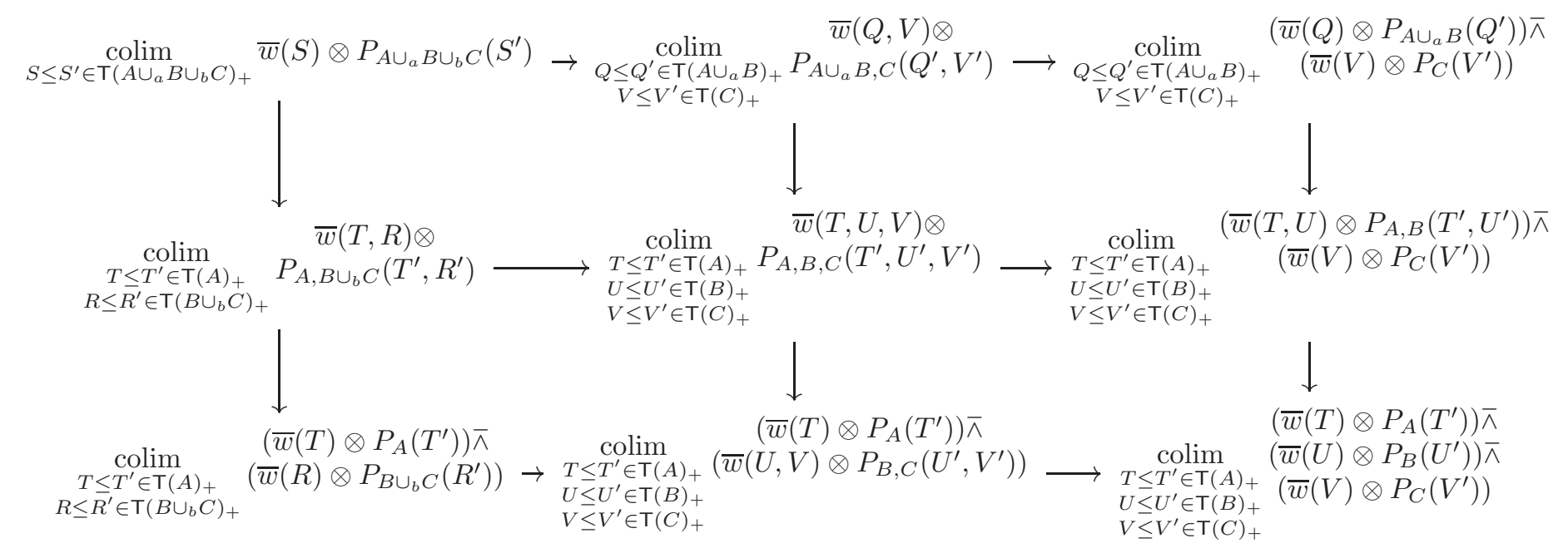

Figure 11: The commutative diagram that verifies the first associativity axiom for the cooperad structure on $B(P)$ in the proof of Theorem 4.29 
category $\mathcal{C}^{\text {op }}$. The cobar construction on $Q$ is then defined to be the bar construction on $Q^{\mathrm{op}}$. This bar construction is a cooperad in $\mathcal{C}^{\text {op }}$ and hence an operad in $\mathcal{C}$. In symbols, the cobar construction on $Q$ is

$$
\Omega(Q):=B\left(Q^{\mathrm{op}}\right)^{\mathrm{op}} \text {. }
$$

It can be useful to have a more explicit description of this.

Definition 5.1 (Cobar construction on a cooperad) The cobar construction, being dual to the bar construction, is defined as an end rather than a coend. Let $Q$ be a cooperad in $\mathcal{C}$. Then for each finite set $A, Q$ determines a functor

$$
Q_{A}(-): \mathrm{T}(A) \rightarrow \mathcal{C}
$$

by

$$
Q_{A}(T)=Q\left(i\left(v_{1}\right)\right) \bar{\wedge} \cdots \bar{\wedge} Q\left(i\left(v_{n}\right)\right)
$$

where $v_{1}, \ldots, v_{n}$ are the vertices of $T$. This is a functor because the cocomposition maps for $Q$ give us maps

$$
Q_{A}(T / e) \rightarrow Q_{A}(T) .
$$

(Recall that the corresponding functor for an operad was defined on $\mathrm{T}(A)^{\mathrm{op}}$.) The cobar construction $\Omega(Q)$ is then the symmetric sequence with

$$
\Omega(Q)(A):=\operatorname{Map}_{\mathbf{T}(A)}\left(\bar{w}(-), Q_{A}(-)\right)=\int_{T \in \mathrm{T}(A)} \operatorname{Map}_{\mathcal{C}}\left(\bar{w}(T), Q_{A}(T)\right) .
$$

This is the end of the bifunctor

$$
\mathrm{T}(A)^{\mathrm{op}} \times \mathrm{T}(A) \rightarrow \mathcal{C}
$$

given by

$$
(T, U) \mapsto \operatorname{Map}_{\mathcal{C}}\left(\bar{w}(T), Q_{A}(U)\right)
$$

where $\operatorname{Map}_{\mathcal{C}}$ denotes the cotensoring structure for $\mathcal{C}$ over $\mathcal{T}$ (and hence the tensoring structure for $\mathcal{C}^{\mathrm{op}}$ ).

Remark 5.2 The cobar construction $\Omega(Q)$ on a reduced cooperad $Q$ in based spaces is isomorphic to the totalization of a cosimplicial cobar construction that is dual to the simplicial bar construction. The terms in this cosimplicial construction are iterated versions of the dual composition product of Remark 2.20] The fact that $\Omega(Q)$ is the totalization of this is dual to the result that $B(P)$ is the realization of the simplicial bar construction.

The operad structure maps for $\Omega(Q)$ are dual to the cooperad maps for $B(P)$. The following result is the dual of Proposition 4.29 .

Corollary 5.3 Let $Q$ be a reduced cooperad in a symmetric monoidal $\mathcal{T}$ category $\mathcal{C}$. Then the cobar construction $\Omega(Q)$ is a reduced operad in $\mathcal{C}$. 


\section{Duality for operads and cooperads}

In this section we examine how the bar and cobar constructions relate to the 'duality' functor

$$
\mathbb{D}: \mathcal{T}^{\text {op }} \rightarrow \mathcal{C} ; X \mapsto \operatorname{Map}_{\mathcal{C}}(X, S)
$$

where $S$ is the unit of the symmetric monoidal structure on $\mathcal{C}$. The case to keep in mind is $\mathcal{C}=\mathcal{S} p$ in which case $S$ is the sphere spectrum and this duality functor is Spanier-Whitehead duality.

Lemma 6.1 Let $Q$ be a cooperad of based spaces. Then $\mathbb{D} Q$ is an operad in the category $\mathcal{C}$.

Proof The composition maps for $\mathbb{D} Q$ are given by

$$
\begin{aligned}
\operatorname{Map}_{\mathcal{C}}(Q(A), S) \pi \operatorname{Map}_{\mathcal{C}}(Q(B), S) & \rightarrow \operatorname{Map}_{\mathcal{C}}(Q(A) \wedge Q(B), S) \\
& \rightarrow \operatorname{Map}_{\mathcal{C}}\left(Q\left(A \cup_{a} B\right), S\right) .
\end{aligned}
$$

The first map is the natural transformation constructed in Proposition 1.12 (it's the distributive map $d$ for $\mathcal{C}^{\text {op }}$ ). The second comes from the corresponding cocomposition map for $Q$.

Remark 6.2 The dual of an operad need not in general be a cooperad because the map $d$ need not in general have an inverse. However when it does we have a nice duality result connecting the bar and cobar constructions. For this to work we need to put the following condition on the spaces that make up our operad.

Definition 6.3 Two based spaces $X, Y$ are compatibly dualizable in $\mathcal{C}$ if the map

$$
d: \operatorname{Map}_{\mathcal{C}}(X, S) \bar{\wedge} \operatorname{Map}_{\mathcal{C}}(Y, S) \rightarrow \operatorname{Map}_{\mathcal{C}}(X \wedge Y, S)
$$

is an isomorphism.

Proposition 6.4 Let $P$ be an operad in based spaces whose terms (that is, the $P(A)$ for finite sets $A$ ) are pairwise compatibly dualizable. Then $\mathbb{D} P$ has a natural cooperad structure. Moreover, we have an isomorphism

$$
\mathbb{D} B(P) \cong \Omega(\mathbb{D} P)
$$

of operads in $\mathcal{C}$. 
Proof The cooperad structure maps for $\mathbb{D} P$ are constructed in the same way as the operad structure maps for $\mathbb{D} Q$ in 6.1 but using the inverse of the relevant map $d$ provided by the 'compatibly dualizable' hypothesis.

The second part relies on the descriptions of the bar and cobar constructions as coends and ends respectively. The coend $B(P)$ is a colimit:

$$
B(P)(A)=\underset{T \leq T^{\prime}}{\operatorname{colim}} \bar{w}(T) \wedge P_{A}\left(T^{\prime}\right)
$$

where the colimit is taken over all inequalities of trees in $\mathrm{T}(A)$. Therefore

$$
\begin{aligned}
\mathbb{D} B(P)(A) & =\operatorname{Map}_{\mathcal{C}}\left(\operatorname{colim} \bar{w}(T) \wedge P_{A}\left(T^{\prime}\right), S\right) \\
& \cong \lim \operatorname{Map}_{\mathcal{C}}\left(\bar{w}(T) \wedge P_{A}\left(T^{\prime}\right), S\right) \\
& \cong \lim \operatorname{Map}_{\mathcal{C}}\left(\bar{w}(T), \operatorname{Map}_{\mathcal{C}}\left(P_{A}\left(T^{\prime}\right), S\right)\right) \\
& \cong \lim \operatorname{Map}_{\mathcal{C}}\left(\bar{w}(T),(\mathbb{D} P)_{A}\left(T^{\prime}\right)\right)
\end{aligned}
$$

The last identity again uses the 'compatibly dualizable' hypothesis in the form $\operatorname{Map}_{\mathcal{C}}\left(P\left(i\left(v_{1}\right)\right) \wedge \ldots \wedge P\left(i\left(v_{n}\right)\right), S\right) \cong \operatorname{Map}_{\mathcal{C}}\left(P\left(i\left(v_{1}\right)\right), S\right) \bar{\wedge} \ldots \bar{\wedge} \operatorname{Map}_{\mathcal{C}}\left(P\left(i\left(v_{n}\right)\right), S\right)$. The final line of this calculation is precisely the limit that defines $\Omega(\mathbb{D} P)$. We leave the reader to check that this is an isomorphism of operads.

Remark 6.5 The only case of this result we will use in this paper is when all the terms of the operad $P$ are $S^{0}$. These are pairwise compatibly dualizable in any $\mathcal{C}$ because

$$
\operatorname{Map}_{\mathcal{C}}\left(S^{0}, C\right) \cong C
$$

for any $C \in \mathcal{C}$.

Remark 6.6 Replacing $\mathcal{C}$ with $\mathcal{C}^{\text {op }}$ we obtain dual results. These concern the functor $\mathbb{S}: X \mapsto X \otimes S$, the 'suspension spectrum' functor. We find that if $Q$ is a cooperad in based spaces then $\mathbb{S} Q$ is a cooperad in $\mathcal{C}$. If $P$ is an operad whose terms are pairwise compatibly dualizable then $\mathbb{S} P$ is an operad in $\mathcal{C}$ and $\mathbb{S} B(P) \cong B(\mathbb{S} P)$.

We have now reached the stage where we can apply our constructions to Goodwillie's calculus of functors (see Section 8). Before doing so, we extend our bar and cobar constructions to modules and comodules. This will then allow us to construct modules over the derivatives of the identity. 


\section{Bar constructions for modules and comodules}

In this section we extend the bar and cobar constructions to modules and comodules. We show that there is a bar construction on left (respectively right) modules over a reduced operad $P$ that yields left (respectively right) comodules over the cooperad $B(P)$. Dually, there is a cobar construction on left (respectively right) comodules over a reduced cooperad $Q$ that yields left (respectively right) modules over the operad $\Omega(Q)$. These are special cases of two-sided bar and cobar constructions. Given a reduced operad $P$ with right module $R$ and left module $L$, we will define a two-sided bar construction $B(R, P, L)$. Taking either $R$ or $L$ to be the unit symmetric sequence $I$ will yield the promised one-sided constructions for individual modules. The two-sided construction is isomorphic to the standard simplicial two-sided bar construction (see Definition (7.9) but, in order to get the comodule structure, we have reinterpreted this in terms of trees.

Most of the material in this section is a straightforward generalization of that of Sections 3 .5 First, in Section 7.1 we describe the more general species of tree necessary for the definitions of the two-sided constructions. In Section $\mathbf{7 . 2}$ we give these definitions and show that the bar construction of Section 4.1 is a special case. In Section 7.3 we construct the maps that make the bar construction on a module into a comodule, and dually, the cobar construction on a comodule into a module.

As previously, $\mathcal{C}$ denotes a symmetric monoidal $\mathcal{T}$-category with null object $*$ and which has all necessary limits and colimits.

\subsection{Generalized trees}

To accommodate the presence of the $P$-modules $R$ and $L$ in the two-sided bar construction, we need to make two changes to our notion of tree, one at the root level and one at the leaf level:

(1) We allow the root element of a tree to have more than one incoming edge.

(2) We allow the leaves of a tree to have repeated labels, that is, an $A-$ labelling is a surjection from $A$ to the set of leaves, rather than a bijection.

We will refer to this notion as a 'generalized tree', or sometimes just a 'tree' if the context makes it clear that we mean the generalized version. The following definition makes things precise. 
Definition 7.1 Let $A$ be a finite set. A generalized $A$-labelled tree consists of

- a poset $T$ with a unique minimal element $r$ (the root) satisfying conditions (2) and (3) of Definition [3.1] and

- a surjection $\iota$ from the finite set $A$ to the set of maximal elements (the leaves) of $T$.

We use letters $T, U, \ldots$ to denote generalized trees, usually taking the labelling map $\iota$ for granted. We write $\operatorname{Tree}(A)$ for the set of isomorphism classes of generalized $A$-labelled trees. All the terminology of Definition 3.1 applies equally well to generalized trees.

Edge collapse for generalized trees is defined in exactly the same way as for the trees of Section 3 except that now we allow ourselves to collapse root edges as well as internal edges. To get the right category structure on $\operatorname{Tree}(A)$ we need a way to collapse leaf edges as well. The following definition provides this.

Definition 7.2 (Bud collapse) A bud in a generalized tree $T$ is a vertex all of whose incoming edges are leaf edges. Equivalently, a bud is a maximal vertex. If $b$ is a bud in $T$, a $b$-leaf is a leaf of $T$ that is attached to $b$.

Given a generalized $A$-labelled tree $T$ and a bud $b \in T$, we define a generalized $A$-labelled tree $T_{b}$ which is obtained from $T$ by bud collapse. The underlying poset of $T_{b}$ is obtained from $T$ by removing the $b$-leaves. This makes $b$ into a leaf in $T_{b}$. The $A$-labelling on $T_{b}$ is that of $T$ for the leaves that still remain, with $b$ inheriting the labels of its old leaves. Formally, we are composing the $A$-labelling on $T$ with the surjection from the leaves of $T$ to the leaves of $T_{b}$ that sends the $b$-leaves in $T$ to $b$. Visually, we can think of this process as collapsing all the leaf edges attached to $b$ (see Figure 12).

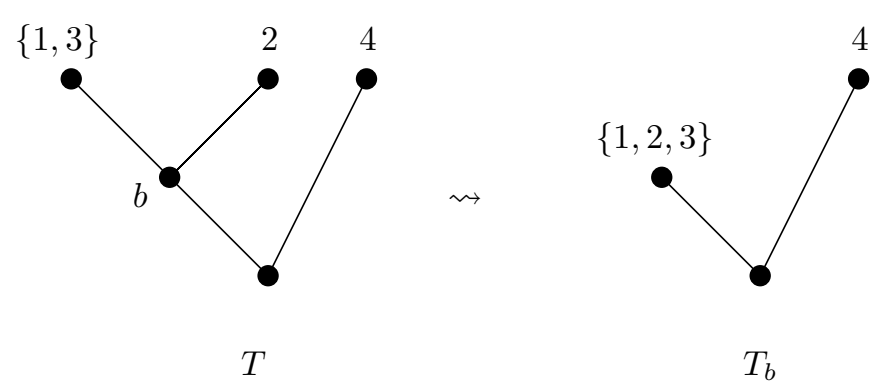

Figure 12: An example of bud collapse for generalized $\{1,2,3,4\}$-labelled trees 
Definition 7.3 (The categories Tree $(A)$ ) If $T$ and $T^{\prime}$ are generalized $A-$ labelled trees, we say that $T \leq T^{\prime}$ if $T$ can be obtained from $T^{\prime}$ by a sequence of edge collapses (of either internal or root edges) or bud collapses. This makes the set $\operatorname{Tree}(A)$ of isomorphism classes of generalized $A$-labelled trees into a poset and hence a category. Standard $A$-labelled trees (as defined in Section (3) are also generalized $A$-labelled trees and $\mathrm{T}(A)$ is a full subcategory of $\operatorname{Tree}(A)$. See Figure 13 for pictures of Tree(1) and Tree(2).

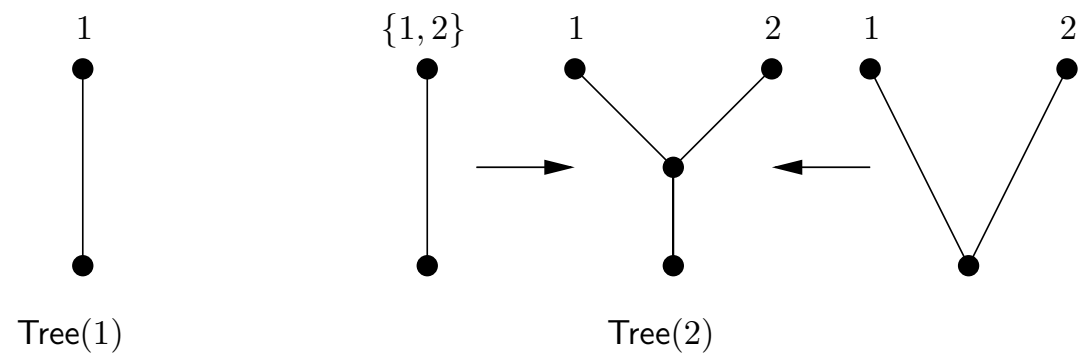

Figure 13: Tree(1) and Tree(2) (the arrows represent the direction of the morphisms in $\operatorname{Tree}(2))$

Definition 7.4 We don't need to change the definition of a weighting for generalized trees: it is an assignment of lengths to the edges of a tree such that the root-leaf distances all equal 1. As before, we write $w(T)$ for the space of weightings on the generalized tree $T$. The following result generalizes Lemma 3.9

Lemma 7.5 Let $T$ be a generalized $A$-labelled tree with $n$ (internal) vertices. Then $w(T)$ is homeomorphic to $D^{n}$ and the boundary $\partial w(T) \cong S^{n-1}$ consists of those points in which some edge of $T$ has length zero.

Proof The labelling plays no role in the space of weightings so we can ignore it. Picture $T$ as a collection of (non-generalized) trees $T_{1}, \ldots, T_{k}$ attached at their roots. Suppose $T_{j}$ has $n_{j}$ vertices so that $n=\sum n_{j}$. Then we have

$$
w(T) \cong w\left(T_{1}\right) \times \cdots \times w\left(T_{k}\right) \cong D^{n_{1}} \times \cdots \times D^{n_{k}} \cong D^{n} .
$$

Under this decomposition, a point is in the boundary of $w(T)$ if and only if any of it is in the boundary of any of the $w\left(T_{j}\right)$. That is, if and only if any of the edges of $T$ has length zero. 
Definition 7.6 The 'space of weightings' functor $w(-): \mathrm{T}(A) \rightarrow \mathcal{U}$ of Definition 3.10 can be extended to all of $\operatorname{Tree}(A)$. To do this, we have to say what happens when we apply $w(-)$ to a morphism $T_{b} \rightarrow T$ coming from a bud collapse (for $b$ a bud in a tree $T$ ). Given a weighting of $T_{b}$ we get a weighting of $T$ by giving length zero to all the leaf edges attached to $b$. This defines a map

$$
w\left(T_{b}\right) \rightarrow w(T)
$$

and it is not hard to see that this does indeed give us a functor

$$
w(-): \operatorname{Tree}(A) \rightarrow \mathcal{U}
$$

as claimed. Adding a disjoint basepoint we get a functor

$$
w(-)_{+}: \operatorname{Tree}(A) \rightarrow \mathcal{T} .
$$

\subsection{The two-sided bar construction}

We now update Definition 4.4 to the two-sided case. Along with the spaces of weightings the key parts of this definition were functors

$$
P_{A}(-): \mathrm{T}(A)^{\mathrm{op}} \rightarrow \mathcal{C} .
$$

The appropriate generalizations of these to functors on Tree $(A)^{\mathrm{op}}$ are as follows.

Definition 7.7 Let $P$ be a reduced operad in $\mathcal{C}$ with right module $R$ and left module $L$. We define functors $(R, P, L)_{A}: \operatorname{Tree}(A)^{\mathrm{op}} \rightarrow \mathcal{C}$ by 11

$$
(R, P, L)_{A}(T):=R(i(r)) \overline{\bigwedge_{\text {vertices } v \in T}} P(i(v)) \overline{\bigwedge_{\text {leaves }} l \in T} L\left(\iota^{-1} l\right) .
$$

Recall that $i(v)$ denotes the set of incoming edges to a vertex $v \in T$. Here $\iota$ denotes the labelling surjection from $A$ to the set of leaves of $T$, so that $\iota^{-1} l$ is the set of labels attached to the leaf $l$.

To complete the definition, we have to give the effect of $(R, P, L)_{A}(-)$ on morphisms in $\operatorname{Tree}(A)$. Notice that $\operatorname{Tree}(A)$ is generated by the morphisms corresponding to

(1) collapse of root edges,

(2) collapse of internal edges, and

(3) bud collapse.

\footnotetext{
${ }^{11}$ It is a serendipitous fact of our terminology for trees that the right module $\mathbf{R}$ relates to the roots of our trees and the left module $\mathbf{L}$ relates to the leaves.
} 
We will describe the effect of $(R, P, L)_{A}(-)$ on each of these types of generating morphism and then check that they are compatible.

(1) Suppose first that $e$ is a root edge of the generalized $A$-labelled tree $T$. Then we have a morphism $T / e \rightarrow T$ that collapses $e$. The corresponding morphism

$$
(R, P, L)_{A}(T) \rightarrow(R, P, L)_{A}(T / e)
$$

is given by the map

$$
R(i(r)) \bar{\wedge} P(i(v)) \rightarrow R(i(r \circ v))
$$

that comes from the right $P$-module structure on $R$. Here $v$ is the upper endpoint of the edge $e$ in $T$. Notice that $r \circ v$ is the root element in $T / e$.

(2) Now suppose that $e$ is an internal edge of $T$. The morphism

$$
(R, P, L)_{A}(T) \rightarrow(R, P, L)_{A}(T / e)
$$

is then given (as in Definition 4.4) by the partial composition map

$$
P(i(u)) \bar{\wedge} P(i(v)) \rightarrow P(i(u \circ v))
$$

for the operad $P$ where $u, v$ are the endpoints of $e$.

(3) Finally, suppose that $b$ is a bud in the generalized $A$-labelled tree $T$. The required map

$$
(R, P, L)_{A}(T) \rightarrow(R, P, L)_{A}\left(T_{b}\right)
$$

comes from the map

$$
P(i(b)) \bar{\wedge} L\left(\iota^{-1} l_{1}\right) \bar{\wedge} \cdots \bar{\wedge} L\left(\iota^{-1} l_{r}\right) \rightarrow L\left(\iota_{1}^{-1} b\right)
$$

that is part of the left $P$-module structure on $L$. Here $l_{1}, \ldots, l_{r}$ are the $b$-leaves in $T$ and we have

$$
\iota^{-1} b=\coprod_{i=1}^{r} \iota^{-1} l_{i}
$$

from the definition of bud collapse, where $\iota_{1}$ is the $A$-labelling of $T_{b}$.

The associativity conditions for $P$ to be an operad and for $R$ and $L$ to be $P$ modules ensure that these choices indeed determine a functor Tree $(A)^{\mathrm{op}} \rightarrow \mathcal{C}$.

Definition 7.8 (Two-sided bar construction) Let $P$ be a reduced operad in $\mathcal{C}$ with right module $R$ and left module $L$ as above. The bar construction on $P$ with coefficients in $R$ and $L$ is the symmetric sequence $B(R, P, L)$ defined by the coends

$$
B(R, P, L)(A):=\int^{T \in \operatorname{Tree}(A)} w(T)_{+} \otimes(R, P, L)_{A}(T)
$$


for finite sets $A$. A bijection $A \rightarrow A^{\prime}$ determines an isomorphism of categories $\operatorname{Tree}(A) \rightarrow \operatorname{Tree}\left(A^{\prime}\right)$ under which the pairs of functors $w_{A}(-), w_{A^{\prime}}(-)$ and $(R, P, L)_{A},(R, P, L)_{A^{\prime}}$ correspond. It therefore induces an isomorphism

$$
B(R, P, L)(A) \rightarrow B(R, P, L)\left(A^{\prime}\right) .
$$

So we obtain a symmetric sequence $B(R, P, L)$.

There is a more informal description of this bar construction that generalizes that of $B(P)$ from Definition 4.1. For a finite set $A$, a point in $B(R, P, L)(A)$ consists of

- a weighted generalized $A$-labelled tree $T$,

- a point in $R(i(r))$ where $r$ is the root of $T$,

- a point in $P(i(v))$ for each vertex $v \in T$, and

- a point in $L\left(\iota^{-1} l\right)$ for each leaf $l \in T$.

These are subject to identifications that tell us what happens when the lengths of some of the edges tend to zero. When a root edge tends to zero we use the right $P$-module structure map for $R$. When an internal edge tends to zero we use the composition map for $P$. When a collection of leaf edges attached to a bud tend to zero (note that the leaf edges attached to a particular bud must all have the same length in a weighting) we use the left $P$-module structure for $L$. Finally, of course, we identify to the basepoint in $B(R, P, L)(A)$ if any of the chosen points in $R(i(r)), P(i(v)), L\left(\iota^{-1} l\right)$ are the basepoint there.

We now recall the simplicial version of the two-sided bar construction for an operads and modules over them.

Definition 7.9 (Simplicial two-sided bar construction) Let $P$ be an operad in $\mathcal{C}$ with right module $R$ and left module $L$. The simplicial bar construction on $P$ with coefficients in $L$ and $R$ is the simplicial object $\mathcal{B}_{\bullet}(R, P, L)$ in the category of symmetric sequences in $\mathcal{C}$ with

$$
\mathcal{B}_{n}(R, P, L):=R \circ \underbrace{P \circ \cdots \circ P}_{n} \circ L \text {. }
$$

The face maps

$$
d_{i}: \mathcal{B}_{n}(R, P, L) \rightarrow \mathcal{B}_{n-1}(R, P, L)
$$

for $i=1, \ldots, n-1$ are given by the operad composition map $P \circ P \rightarrow P$ applied to the $i^{\text {th }}$ and $i+1^{\text {th }}$ factors. The face map $d_{0}$ is given by the right module 
structure $R \circ P \rightarrow R$ and $d_{n}$ is given by the left module structure $P \circ L \rightarrow L$. The degeneracy map

$$
s_{j}: \mathcal{B}_{n}(R, P, L) \rightarrow \mathcal{B}_{n+1}(R, P, L)
$$

is given by using the unit map $I \rightarrow P$ to insert an extra copy of $P$ between the $j^{\text {th }}$ and $j+1^{\text {th }}$ factors.

Proposition 7.10 Let $P$ be a reduced operad in $\mathcal{C}$ with right module $R$ and left module $L$. The bar construction of Definition 7.8 is isomorphic to the geometric realization of the simplicial bar construction:

$$
B(R, P, L) \cong\left|\mathcal{B}_{\bullet}(R, P, L)\right|
$$

Proof This is a straightforward extension of the argument used to prove Proposition 4.13

Our first example of the two-sided bar construction is that the reduced bar construction a lone operad is a special case.

Example 7.11 Let $P$ be a reduced operad in $\mathcal{C}$ and take $R=L=I$ the unit symmetric sequence. Recall that $I$ is a left and right module over any augmented operad. It is easy to see from the definitions that for the simplicial bar constructions we have

$$
\mathcal{B}_{\bullet}(I, P, I) \cong \mathcal{B}_{\bullet}(P)
$$

This tells us that

$$
B(I, P, I) \cong B(P),
$$

but we can see this directly as well. First notice that

$$
(I, P, I)_{A}(T) \cong \begin{cases}P_{A}(T) & \text { if } T \in \mathrm{T}(A) ; \\ * & \text { otherwise }\end{cases}
$$

The reason for this is as follows. Because $I(n)=*$ for $n>1$, we have $(I, P, I)_{A}(T)=*$ whenever $T$ has more than one root edge, or when any leaf has more than one label. These are precisely the generalized $A$-labelled trees not in $\mathrm{T}(A)$. For $T \in \mathrm{T}(A)$ we have

$$
(I, P, I)_{A}(T)=I(1) \bar{\wedge} P_{A}(T) \bar{\wedge} I(1) \bar{\wedge} \cdots \bar{\wedge} I(1) \cong P_{A}(T) .
$$

This calculation means that only the objects $T \in \mathrm{T}(A)$ contribute to the calculation of the coend in Definition [7.8. However, we still have to take into 
account morphisms $U \rightarrow T$ with $U \notin \mathrm{T}(A)$. This amounts to collapsing to the basepoint those weighted trees in which either the root edge or a leaf edge has length zero (since these are the images of the maps $w(U) \rightarrow w(T)$ ). All together this tells us that $B(I, P, I)(A)$ is equal to the coend

$$
\int^{T \in \mathbf{T}(A)} \bar{w}(T) \otimes P_{A}(T)
$$

where $\bar{w}(T)$ is the quotient of $w(T)$ by the weightings which have either root or leaf edge of length zero. This is precisely $B(P)(A)$. Therefore we have $B(I, P, I) \cong B(P)$ as claimed.

Example 7.12 It is easy to see that $B(R, P, L)(1)=R(1) \bar{\wedge} L(1)$. We have already seen (Figure 13) that there are three objects in Tree(2). From this we see that $B(R, P, L)(2)$ is the homotopy pushout of the following diagram

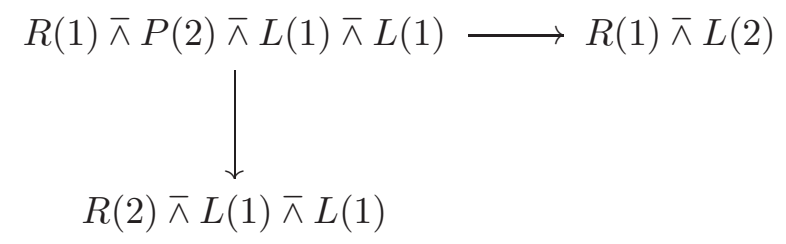

If $R=L=I$, the bottom-left and top-right objects are $*$ and the top-left object is $P(2)$. So we recover

$$
B(P)(2)=B(I, P, I)(2)=\Sigma P(2) .
$$

Definition 7.13 (Bar constructions for modules) Let $P$ be a reduced operad in $\mathcal{C}$ and let $R$ be a right $P$-module. We define the bar construction on $R$ by

$$
B(R):=B(R, P, I)
$$

where $I$, as previously, is the unit for the composition product of symmetric sequences. If $L$ is a left $P$-module, its bar construction is

$$
B(L):=B(I, P, L) .
$$

We trust that it will not be confusing to use the same notation for the bar construction of right and left modules.

Example 7.14 Applying Example 7.12 to the one-sided case we see that

$$
B(R)(1) \cong R(1) ; \quad B(R)(2) \cong \operatorname{hocofib}(R(1) \bar{\wedge} P(2) \rightarrow R(2))
$$

and

$$
B(L)(1) \cong L(1) ; \quad B(L)(2) \cong \operatorname{hocofib}(P(2) \pi L(1) \bar{\wedge} L(1) \rightarrow L(2)) .
$$


Definition 7.15 (Cobar constructions for comodules) All the constructions of this section can be applied to operads and modules in $\mathcal{C}^{\mathrm{op}}$, that is, to cooperads and comodules in $\mathcal{C}$. We summarize the results.

If $Q$ is a reduced cooperad in $\mathcal{C}$ with left comodule $L$ and right comodule $R$, the formula of Definition 7.7 defines functors

$$
(R, Q, L)_{A}(-): \operatorname{Tree}(A) \rightarrow \mathcal{C}
$$

for each finite set $A$ and we define the cobar construction on $Q$ with coefficients in $R$ and $L$ to be the symmetric sequence $\Omega(R, Q, L)$ with

$$
\Omega(R, Q, L)(A):=\int_{T \in \operatorname{Tree}(A)} \operatorname{Map}_{\mathcal{C}}\left(w(T)_{+},(R, Q, L)_{A}(T)\right) .
$$

This is isomorphic to the totalization of the two-sided cosimplicial cobar construction on $Q$ with coefficients in $R$ and $L$. The cobar construction on $R$ is

$$
\Omega(R):=\Omega(R, Q, I)
$$

and the cobar construction on $L$ is

$$
\Omega(L):=\Omega(I, Q, L) .
$$

Example 7.16 Taking $R=L=I$ we recover the cobar construction of Section 5 .

$$
\Omega(I, Q, I) \cong \Omega(Q) .
$$

Example 7.17 Taking the duals of the results of Example 7.12 we see that

$$
\Omega(R, Q, L)(1) \cong R(1) \bar{\wedge} L(1)
$$

and that $\Omega(R, Q, L)(2)$ is the homotopy pullback of

$$
\begin{aligned}
& R(1) \bar{\wedge} L(2) \\
& R(2) \bar{\wedge} L(1) \bar{\wedge} L(1) \longrightarrow R(1) \bar{\wedge} Q(2) \bar{\wedge} L(1) \bar{\wedge} L(1) .
\end{aligned}
$$

In particular,

$$
\Omega(R)(1) \cong R(1) ; \quad \Omega(R)(2) \cong \operatorname{hofib}(R(2) \rightarrow R(1) \wedge Q(2))
$$

and

$$
\Omega(L)(1) \cong L(1) ; \quad \Omega(L)(2) \cong \operatorname{hofib}(L(2) \rightarrow Q(2) \bar{\wedge} L(1) \bar{\wedge} L(1)) .
$$




\subsection{Structure maps for bar constructions on modules}

In this section we use similar methods to Section 4.3 to show that the bar construction on a $P$-module (that is, a single left or right module) is a comodule over the cooperad $B(P)$. In fact, we will construct maps of the form

$$
B(R, P, L) \rightarrow B(R, P, I) \widehat{o} B(I, P, L)
$$

where $\widehat{o}$ is the composition of symmetric sequences defined using the product in $\mathcal{C}$ rather than the coproduct (see Remark 2.20). Taking $R=I$ and recalling that $B(I, P, I)=B(P)$ we obtain a left $B(P)$-comodule structure on $B(L)=$ $B(I, P, L)$. Similarly, taking $L=I$ we get a right $B(P)$-comodule structure on $B(R)$. Notice that taking $R=L=I$ we recover the cooperad structure on $B(P)$.

The definition of the map (7.18) is a relatively straightforward generalization of the cooperad structure on $B(P)$. We start by describing the grafting and ungrafting processes for generalized trees.

Definition 7.19 (Grafting for generalized trees) Let $T$ be a generalized $A$ labelled tree and $U$ a generalized $B$-labelled tree and let $a$ be an element of $A$. We will define the grafting of $U$ onto $T$ only if $T$ and $U$ satisfy the following conditions:

- The root of $U$ has only one incoming edge.

- The leaf of $T$ labelled by $a$ is labelled only by $a$ and no other elements of $A$.

In this case, the grafted tree $T \cup_{a} U$ is defined exactly as in Definition 4.14 by identifying the root edge of $U$ to the $a$-leaf edge of $T$. Figure 14 gives an example.
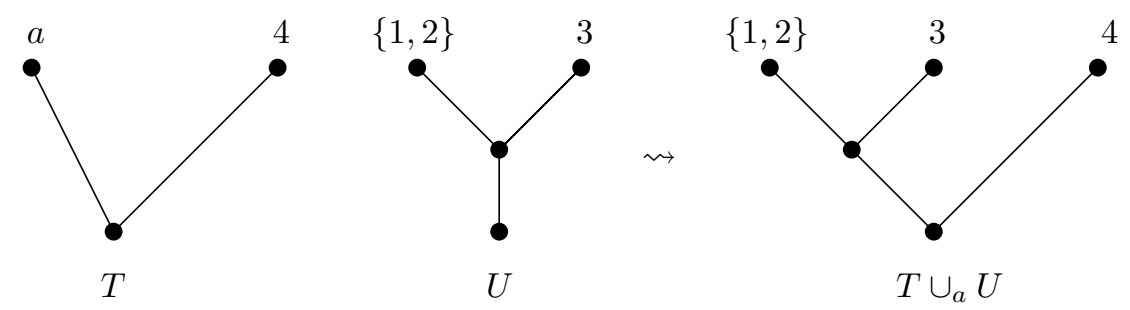

Figure 14: Grafting generalized labelled trees 
To define the maps (7.18) we will need to graft trees onto all of the leaf edges of the base tree $T$. To do this, we must assume that all the leaves of $T$ only have one label, so that $T$ satisfies the stronger condition for a labelling we required in Definition [3.3 Notice also that the trees $U$ we are to graft onto $T$ satisfy the stronger root condition of Definition 3.1. The following definitions will help us talk about trees of these types.

Definition 7.20 For a finite set $A$, we define the following full subcategories of $\operatorname{Tree}(A)$ :

$$
\begin{gathered}
\mathrm{T}_{\text {root }}(A):=\{T \in \operatorname{Tree}(A) \mid \text { the root of } T \text { has only one incoming edge }\} \\
\mathrm{T}_{\text {leaf }}(A):=\{T \in \operatorname{Tree}(A) \mid \text { the leaves of } T \text { are labelled bijectively by } A\} .
\end{gathered}
$$

Notice that $\mathrm{T}(A)=\mathrm{T}_{\text {root }}(A) \cap \mathrm{T}_{\text {leaf }}(A)$.

Definition 7.21 Let $A=\coprod_{j \in J} A_{j}$ be a partition of $A$ into nonempty subsets. Given trees $U_{j} \in \mathrm{T}_{\text {root }}\left(A_{j}\right)$ and $T \in \mathrm{T}_{\text {leaf }}(J)$, we denote the tree obtained by grafting all the $U_{j}$ onto $T$ at the appropriate places by

$$
T \cup_{J} U_{j} \text {. }
$$

We say that a generalized $A$-labelled tree is of type $\left\{A_{j}\right\}$ if it is of the form $T \cup_{J} U_{j}$ for some such $T$ and $U_{j}$. The correct generalization of the functor of Proposition 4.21 is then a functor

$$
\operatorname{Tree}(A)_{+} \rightarrow \mathrm{T}_{\text {leaf }}(J)_{+} \wedge \mathrm{T}_{\text {root }}\left(A_{j_{1}}\right)_{+} \wedge \ldots \wedge \mathrm{T}_{\text {leaf }}\left(A_{j_{r}}\right)_{+}
$$

that breaks the tree $\left(T \cup_{J} U_{j}\right)$ into its components $T$ and the $U_{j}$ and sends a tree not of type $\left\{A_{j}\right\}$ to the initial object on the right-hand side. This 'ungrafting' functor is the basis of the map (7.18).

Our new categories of trees can be used as the base categories for defining the one-sided bar constructions. For this we need the appropriate spaces of weightings.

Definition 7.22 For each finite set $A$ we define a functor

$$
w_{\text {leaf }}(-): \mathrm{T}_{\text {leaf }}(A) \rightarrow \mathcal{T}
$$

where $w_{\text {leaf }}(T)$ is the quotient of $w(T)$ by the space of weightings in which some leaf edge has length zero, and a functor

$$
w_{\text {root }}(-): \mathrm{T}_{\text {root }}(A) \rightarrow \mathcal{T}
$$

where $w_{\text {root }}(T)$ is the quotient of $w(T)$ by the space of weightings in which the root edge has length zero. 
Lemma 7.23 Let $P$ be a reduced operad in $\mathcal{C}$ with right module $R$ and left module $L$. Then the one-sided bar constructions are given by

$$
B(R)(A)=B(R, P, I)(A) \cong \int^{T \in \mathrm{T}_{\text {leaf }}(A)} w_{\text {leaf }}(T) \otimes(R, P, I)_{A}(T)
$$

and

$$
B(L)(A)=B(I, P, L)(A) \cong \int^{T \in \mathrm{T}_{\text {root }}(A)} w_{\text {root }}(T) \otimes(I, P, L)_{A}(T) .
$$

Proof These calculations are similar to that in Example 7.11 where we showed that $B(P)=B(I, P, I)$. They use the facts that

$$
(R, P, I)_{A}(T)=* \text { for } T \notin \mathrm{T}_{\text {leaf }}(A)
$$

and

$$
(I, P, L)_{A}(T)=* \text { for } T \notin \mathrm{T}_{\text {root }}(A) .
$$

The final piece of the puzzle is the construction of a map analogous to (4.18) that tells us how to weight the trees obtained from ungrafting.

Definition 7.24 Let $A=\coprod_{j \in J} A_{j}$ be a partition of the finite set $A$ into nonempty subsets. Given trees $T \in \mathrm{T}_{\text {leaf }}(J)$ and $U_{j} \in \mathrm{T}_{\text {root }}\left(A_{j}\right)$ we define a map

$$
w\left(T \cup_{J} U_{j}\right)_{+} \rightarrow w_{\text {leaf }}(T) \wedge w_{\text {root }}\left(U_{j_{1}}\right) \wedge \ldots \wedge w_{\text {root }}\left(U_{j_{r}}\right)
$$

by the obvious generalization of the construction of the maps $\bar{w}\left(T \cup_{a} U\right) \rightarrow$ $\bar{w}(T) \wedge \bar{w}(U)$ in Definition 4.16 .

Definition 7.25 Putting together all these ingredients we construct maps

$$
B(R, P, L)(A) \rightarrow B(R, P, I)(J) \bar{\wedge} B(I, P, L)\left(A_{j_{1}}\right) \bar{\wedge} \cdots \bar{\wedge} B(I, P, L)\left(A_{j_{r}}\right) .
$$

In an analogous way to Definition 4.26] these come from the maps of Definition 7.24 together with the isomorphisms

$(R, P, L)_{A}\left(T \cup_{J} U_{j}\right) \rightarrow(R, P, I)_{J}(T) \bar{\wedge}(I, P, L)_{A_{j_{1}}}\left(U_{j_{1}}\right) \bar{\wedge} \cdots \bar{\wedge}(I, P, L)_{A_{j_{r}}}\left(U_{j_{r}}\right)$.

Together these maps make up the map of symmetric sequences

$$
B(R, P, L) \rightarrow B(R, P, I) \widehat{\circ} B(I, P, L)
$$

as promised.

Proposition 7.26 Let $P$ be a reduced operad in $\mathcal{C}$ with right module $R$ and left module $L$. The maps of Definition 7.25 determine a right $B(P)$-comodule structure on $B(R)$ and a left $B(P)$-comodule structure on $B(L)$. 
Proof Taking $L=I$ in 7.25 we get the right comodule structure on $B(R)$. Taking $R=I$ we get the left comodule structure on $B(L)$. We have to check the appropriate associativity and unit axioms. This is a generalization of the work of Section 4.3. We leave the reader to write out all the details, including the diagrams corresponding to Figure 11.

Corollary 7.27 Dually, suppose that $Q$ is a reduced cooperad in $\mathcal{C}$ with right comodule $R$ and left comodule $L$. Then there is a map

$$
\Omega(R, Q, I) \circ \Omega(I, Q, L) \rightarrow \Omega(R, Q, L)
$$

that makes $\Omega(R)$ into a right $\Omega(Q)$-module (by taking $L=I$ ) and $\Omega(L)$ into a left $\Omega(Q)$-module (by taking $R=I$ ).

Proof Apply Proposition 7.26 to $Q$ considered as an operad in $\mathcal{C}^{\text {op }}$.

This completes our descriptions of the bar and cobar constructions for operads, cooperads, modules and comodules. We turn now to our main application of this theory - the Goodwillie derivatives of the identity functor.

\section{Application to the calculus of functors}

In this section we describe our application of bar and cobar constructions to Goodwillie's calculus of homotopy functors. The main result is that the derivatives of the identity form an operad in spectra. We now assume that $\mathcal{C}$ is a suitable category $\mathcal{S} p$ of spectra, for example, the $S$-modules of EKMM [6] (see Example 1.14(2)).

Let $I: \mathcal{T} \rightarrow \mathcal{T}$ be the identity functor on based spaces. The Goodwillie derivatives of $I$ can be described in terms of the partition poset complexes [1. We recall one of the ways to define these.

Definition 8.1 A partition of a finite set $A$ is an equivalence relation on $A$. Let $K(A)$ be the poset formed by the partitions of $A$ with $\lambda \leq \mu$ if $\lambda$ is finer than $\mu$, that is, if the set of relations for $\lambda$ is contained in the set of relations for $\mu$. The category $K(A)$ has an initial object $\widehat{0}$ and a terminal object $\widehat{1}$. Let $K_{0}(A)=K(A)-\widehat{0}$, the category of proper partitions, and $K_{1}(A)=K(A)-\widehat{1}$, the category of non-trivial partitions. Note that the group $\Sigma_{A}$ of permutations of $A$ acts on all of these categories in an obvious way. 
Definition 8.2 (Partition poset complexes) For a finite set $A$, the partition poset complex $\Delta(A)$ is the geometric realization of the following simplicial set $T(A)$. formed from the nerves of these categories of partitions:

$$
T(A) \bullet=\frac{N_{\bullet} K(A)}{N_{\bullet} K_{0}(A) \cup N_{\bullet} K_{1}(A)}
$$

So the $n$-simplices in $T(A)$ • are sequences of $n+1$ partitions

$$
\lambda_{0} \leq \lambda_{1} \leq \cdots \leq \lambda_{n}
$$

with a sequence identified to the basepoint if it does not have both $\lambda_{0}=\widehat{0}$ and $\lambda_{n}=\widehat{1}$. The face and degeneracy maps are given by respectively removing partitions from the sequence and repeating terms in the usual way for the nerve of a category. The simplicial set $T(A)$. is pointed and so its geometric realization $\Delta(A)$ is a based space. A bijection $A \rightarrow A^{\prime}$ induces an isomorphism $\Delta(A) \rightarrow \Delta\left(A^{\prime}\right)$ that makes $\Delta$ into a symmetric sequence in $\mathcal{T}$.

Remark 8.3 What we are calling the partition poset complex is the suspension of the complex $K_{n}$ of [1]. The simplicial set $T(n) \bullet$ is isomorphic to that called $T_{n}$ in Definition 1.1 of [1].

Proposition 8.4 (Arone-Mahowald, [1]) The derivatives of the identity are modelled by the dual spectra of the finite complexes $\Delta(n)=\Delta(\{1, \ldots, n\})$ :

$$
\partial_{n} I \simeq \operatorname{Map}_{\mathcal{S} p}(\Delta(n), S)
$$

The action of the symmetric group $\Sigma_{n}$ on $\Delta(n)$ induces an action on the dual spectrum and this agrees with the action that comes with the spectrum $\partial_{n} I$.

The key observation (apparently due to Greg Arone) is that the partition poset complexes can be described as spaces of trees. We can interpret these as the spaces of a bar construction.

Definition 8.5 Let $\underline{S^{0}}$ be the operad in based spaces with

$$
\underline{S^{0}}(A):=S^{0}
$$

for all finite sets $A$ and with all composition maps equal to the identity on $S^{0}$. This is the operad for commutative monoids of based spaces.

Lemma 8.6 The partition poset complex $\Delta(A)$ is homeomorphic to the bar construction $B\left(\underline{S^{0}}\right)(A)$. 
Proof We have already seen that $B\left(\underline{S^{0}}\right)$ is homeomorphic to the realization of the simplicial bar construction on $\underline{S^{0}}$. It is therefore enough to show that the simplicial set $T(A)$ • used to define $\Delta(A)$ is also given by this simplicial bar construction.

A non-basepoint $n$-simplex in $T(A)$ is an increasing sequence of partitions of $A$ of length $n-1$. On the other hand the based set of $n$-simplices in the simplicial bar construction is

$$
\underbrace{S^{0} \circ \cdots \circ \underline{S^{0}}}_{n}(A)
$$

But this is equal to the wedge over increasing sequences of partitions of length $n-1$ of $S^{0}$. Hence we see that the two sets of $n$-simplices are the same. The face and degeneracy maps in each case correspond to removing a partition and repeating a partition respectively. We therefore have isomorphic simplicial sets.

Remark 8.7 In [18, Bruno Vallette describes the notion of a $P$-partition for an operad $P$ in Set. The $P$-partitions form a poset whose nerve (or order complex in [18) is isomorphic to the bar construction $B\left(P_{+}\right)$(where we are considering $P$ as a discrete operad in unbased spaces and adding a disjoint basepoint). Lemma 8.6 is the special case of this fact when $P$ is the "commutative operad' in Set, that is, with $P(n)=*$ for all $n$.

Corollary 8.8 Let $\partial_{n} I$ denote the model of the $n^{\text {th }}$ derivative of the identity given by

$$
\partial_{n} I=\operatorname{Map}_{\mathcal{S} p}(\Delta(n), S)
$$

Then we have

$$
\partial_{n} I=\Omega\left(\mathbb{D} \underline{S^{0}}\right)(n) .
$$

In particular, the derivatives of the identity form an operad in spectra. We denote this operad by $\partial_{*} I$.

Proof We have

$$
\partial_{n} I=\operatorname{Map}(\Delta(n), S)=\mathbb{D} B\left(\underline{S^{0}}\right)(n)=\Omega\left(\mathbb{D} \underline{S^{0}}\right)(n)
$$

by Lemma 8.6 and Proposition 6.4 (which applies since all the spaces in $\underline{S^{0}}$ are $\left.S^{0}\right)$. 
Remark 8.9 The derivatives of the identity are the cobar construction on the cooperad $\underline{S}$ in spectra with

$$
\underline{S}(A)=\mathbb{D} \underline{S^{0}}(A)=S
$$

where $S$ is the sphere spectrum, for all finite sets $A$ and with all cocomposition maps the canonical isomorphisms. This is the analogue for spectra of the cooperad for cocommutative coalgebras.

Remark 8.10 We can use the constructions of Section $\mathbf{7}$ to get modules over the operad $\partial_{*} I$. If $C$ is a comodule over $\underline{S}$ then its cobar construction $\Omega(C)$ is a $\partial_{*} I$-module. We give two examples:

(1) Let $X$ be a based space. Then the suspension spectrum $\Sigma^{\infty} X$ is a $\underline{S}_{-}^{-}$ coalgebra (that is, just a commutative coalgebra) with comultiplication given by the (reduced) diagonal map on $X$ :

$$
\Sigma^{\infty} X \rightarrow \Sigma^{\infty}(X \wedge X) \cong \Sigma^{\infty} X \wedge \Sigma^{\infty} X .
$$

As remarked in Definition 2.22 a coalgebra over a cooperad $Q$ determines a left $Q$-comodule. Thus we obtain a left $\underline{S}$-comodule $\underline{\Sigma^{\infty} X}$. We now take the cobar construction to get a left $\partial_{*} I$-module

$$
M_{X}:=\Omega\left(\underline{\Sigma^{\infty} X}\right)=\Omega\left(I, \underline{S}, \underline{\Sigma^{\infty} X}\right)
$$

(where $I$ in this formula denotes the unit symmetric sequence of Definition 2.5). From the calculations of 7.17 we find that

$$
M_{X}(1)=\Sigma^{\infty} X
$$

and

$$
\begin{aligned}
M_{X}(2) & \cong \operatorname{hofib}\left(\Sigma^{\infty} X \rightarrow \Sigma^{\infty} X \wedge \Sigma^{\infty} X\right) \\
& \simeq \Sigma^{-1} \operatorname{hocofib}\left(\Sigma^{\infty} X \rightarrow \Sigma^{\infty} X \wedge \Sigma^{\infty} X\right) \\
& \simeq \Sigma^{-1} \Sigma^{\infty} \operatorname{hocofib}(X \rightarrow X \wedge X)
\end{aligned}
$$

So $M_{X}(2)$ is (up to homotopy and a desuspension) the mapping cone of the reduced diagonal on $X$. Further work is needed to analyze the spectra $M_{X}(n)$ for larger $n$. In Section 9.7 we will look at ways to calculate the homology of these spectra.

(2) A moment's thought will reveal that a right $\underline{S}$-comodule is precisely the same thing as a functor

$$
(\text { FinSets, } \rightarrow) \longrightarrow \mathcal{S} p
$$

where the left-hand side is the category of finite sets with morphisms given by the surjections. Work in progress by Greg Arone has demonstrated a relationship between such functors and the Goodwillie calculus of homotopy functors $F$ from based spaces to spectra. 
Remark 8.11 (Derivatives of general homotopy functors) The derivatives of any homotopy functor $F$ form a symmetric sequence in spectra and it is natural to ask how these symmetric sequences might be related for different functors. We conjecture that there is in general a map of symmetric sequences

$$
\partial_{*} F \circ \partial_{*} G \rightarrow \partial_{*}(F G)
$$

for any two homotopy functors $F, G: \mathcal{T} \rightarrow \mathcal{T}$ such that $F(*)=*$, where $F G$ denotes the composite of $F$ and $G$. These maps should have suitable associativity properties that taking $F=G=I$ would recover an operad structure on $\partial_{*} I$ equivalent to the one we have constructed in this section. Similarly, taking $F=I$ would yield the structure of a left $\partial_{*} I$-module on $\partial_{*} G$ and taking $G=I$ a right $\partial_{*} I$-module structure on $\partial_{*} F$. The main obstacle at present for constructing these maps is finding good models for the derivatives of a general functor in a symmetric monoidal category $\mathcal{S} p$ of spectra. In the case of the identity functor we were fortunate that such models naturally arose from the partition poset complexes.

\section{Homology of the bar and cobar constructions and Koszul duality}

In this section we look at spectral sequences for calculating the homology of the bar and cobar constructions on operads and cooperads in based spaces or spectra. It turns out that we can relate the $E^{1}$-term of these spectral sequences to the algebraic bar and cobar constructions described in, for example, 8 8 and [7. This leads to a link with Koszul duality which says, briefly, that if the homology of the reduced operad $P$ is Koszul, then the homology of $B(P)$ is its Koszul dual cooperad, and dually, if the homology of the cooperad $Q$ is Koszul then the homology of $\Omega(Q)$ is its Koszul dual operad. This supports the point-of-view that the bar construction for an operad in based spaces or spectra is the analogue of the Koszul dual for an algebraic operad.

Here is a summary of this section. We start in Section 9.1 by recalling how the homology (with coefficients in the commutative ring $k$ ) of an operad in based spaces or spectra has the structure of an operad in graded $k$-modules. Then in Section 9.2 the main work of the chapter begins and we describe the filtration of the bar construction that gives rise to our spectral sequence and identify the 'filtration quotients'. This filtration is based on the number of vertices in the trees that underlie the bar construction. We deal immediately with the two-sided construction of Section [7.2 recalling that the construction for a lone 
operad is a special case of this. As usual, for the cobar construction, we just dualize everything. That is, we get a cofiltration, or tower, whose inverse limit is the cobar construction and we identify the fibres of the stages in this tower. In Section 9.3 we give conditions under which the inclusion maps of the filtrations are cofibrations, thus ensuring that our 'filtration quotients' are actually the homotopy cofibres of filtration. This will allow us later to use our identification of these quotients to calculate the $E^{1}$ term in the spectral sequence. This $E^{1}$ term turns out to be given by the algebraic bar construction which we describe in Section 9.4. We give a definition of this that emphasizes its similarity to the topological version and show that this definition is equivalent to that given by Getzler and Jones [8] and Fresse [7. Then in Section 9.5] we finally set up the spectral sequence and identify its $E^{1}$ term with the algebraic bar construction as claimed. In Section 9.6 we look at Koszul operads and prove the result identifying the homology of the bar construction on $P$ with the Koszul dual of the homology of $P$. Finally, in Section 9.7 we use our spectral sequences to investigate the homology of the $\partial_{*} I$-modules $M_{X}$ constructed in Remark $8.10(1)$.

\subsection{Homology of topological operads}

Throughout the chapter we fix a commutative ring $k$ and consider the categories $\operatorname{Mod}_{k}$ of graded $k$-modules and $\mathrm{Ch}_{k}$ of chain complexes over $k$. First we describe the symmetric monoidal structure on these categories.

Definition 9.1 The tensor product determines a symmetric monoidal structure on graded $k$-modules with

$$
(M \otimes N)_{r}:=\bigoplus_{p+q=r} M_{p} \otimes N_{q}
$$

where the graded symmetry isomorphism

$$
M \otimes N \rightarrow N \otimes M
$$

is given by

$$
m \otimes n \mapsto(-1)^{|m||n|} n \otimes m
$$

and the unit object is the graded module $k$ concentrated in degree 0 . If $M$ and $N$ are chain complexes with differentials $d_{M}$ and $d_{N}$ respectively, we define a differential on $M \otimes N$ by

$$
d_{M \otimes N}(m \otimes n):=d_{M}(m) \otimes n+(-1)^{|m|} m \otimes d_{N}(n) .
$$

This makes $\otimes$ into a symmetric monoidal structure on $\mathrm{Ch}_{k}$ with the same unit $k$ endowed with the trivial differential. 
Throughout this section we will use $H_{*}(-)$ to denote the homology with coefficients in the commutative ring $k$ of an object in $\mathcal{C}$ when $\mathcal{C}$ is either $\mathcal{T}$ or $\mathcal{S} p$. If $\mathcal{C}$ is the category $\mathcal{T}$ of based spaces, this is the reduced homology 12 If $\mathcal{C}$ is a category $\mathcal{S} p$ of spectra, it is the spectrum homology $H_{*}(E)=\pi_{*}(H k \wedge E)$. We recall the Künneth maps for these homology theories.

Proposition 9.2 Let $\mathcal{C}=\mathcal{T}$ or $\mathcal{S} p$ and take $C, D \in \mathcal{C}$. Then there is a natural map

$$
H_{*}(C) \otimes H_{*}(D) \rightarrow H_{*}(C \bar{\wedge} D)
$$

that is an isomorphism if either $H_{*}(C)$ or $H_{*}(D)$ consists of flat $k$-modules. These maps are symmetric monoidal in the sense that they commute with the associativity and commutativity isomorphisms in the categories $\mathcal{C}$ and $\operatorname{Mod}_{k}$.

Definition 9.3 Let $M$ be a symmetric sequence in $\mathcal{T}$ or $\mathcal{S} p$. Then we denote by $H_{*} M$ the symmetric sequence of graded $k$-modules given by

$$
H_{*} M(A):=H_{*}(M(A)) .
$$

The main result of this section is that the homology of a topological operad or cooperad is, under suitable conditions, an operad or cooperad in $\operatorname{Mod}_{k}$.

Lemma 9.4 Let $P$ be an operad in $\mathcal{T}$ or $\mathcal{S} p$. Then $H_{*} P$ is an operad of graded $k$-modules. If $P$ is reduced then so is $H_{*} P$. If $M$ is a left (respectively, right) $P$-module, then $H_{*} M$ is a left (respectively, right) $H_{*} P$-module.

Let $Q$ be a cooperad in $\mathcal{T}$ or $\mathcal{S} p$ such that the homology groups $H_{*}(Q(A))$ are flat $k$-modules. Then $H_{*}(Q)$ is a cooperad of graded $k$-modules that is reduced if $Q$ is. If $C$ is a right $Q$-comodule then $H_{*}(C)$ is a right $H_{*}(Q)$-comodule. If $C$ is a left $Q$-comodule such that the $H_{*}(C(A))$ are flat $k$-modules then $H_{*}(C)$ is a left $H_{*}(Q)$-comodule.

Proof The operad structure maps are given by the composites

$$
H_{*}(P(A)) \otimes H_{*}(P(B)) \rightarrow H_{*}(P(A) \wedge P(B)) \rightarrow H_{*}\left(P\left(A \cup_{a} B\right)\right)
$$

and the unit by the map

$$
k \cong H_{*}(S) \rightarrow H_{*}(P)(1)
$$

where $S$ denotes either $S^{0}$, the unit of $\mathcal{T}$, or the unit of $\mathcal{S} p$. To check the operad axioms we use the associativity and commutativity of the Künneth

\footnotetext{
${ }^{12}$ We stress that any homology group of a based space in this paper is meant to be the reduced homology.
} 
formula as stated in Proposition 9.2. Clearly, if $P$ is reduced (so that the unit map $S \rightarrow P(1)$ is an isomorphism) then so is $H_{*} P$. The structure maps for $H_{*} M$ are defined similarly.

In the cooperad case we need the flatness condition. It allows us to define cocomposition maps by

$$
H_{*}\left(Q\left(A \cup_{a} B\right)\right) \rightarrow H_{*}(Q(A) \wedge Q(B)) \cong H_{*}(Q(A)) \otimes H_{*}(Q(B))
$$

using the inverse of the Künneth map. The counit map is the composite

$$
H_{*} Q(1) \rightarrow H_{*}(S) \cong k
$$

and again, if $Q$ is reduced, so is $H_{*} Q$. In the case of a right comodule $C$ we similarly get comodule structure maps

$$
H_{*}\left(C\left(A \cup_{a} B\right)\right) \rightarrow H_{*}(C(A) \wedge Q(B)) \cong H_{*}(C(A)) \otimes H_{*}(Q(B))
$$

where the Künneth map is an isomorphism without any condition on $H_{*}(C(A))$ (we are still assuming that the $H_{*}(Q(B))$ are flat). For a left comodule, we do still need the flatness assumption.

Remark 9.5 We can consider cohomology instead of homology in which case the Künneth isomorphism also requires a finiteness hypothesis. We get the following results. If $Q$ is a cooperad in based spaces or spectra then $H^{*}(Q)$ is an operad of graded $k$-modules. If $P$ is an operad with the cohomology groups $H^{*}(P)$ finitely-generated flat $k$-modules then $H^{*}(P)$ is a cooperad of graded $k$-modules. Similar results hold for comodules and modules.

\subsection{Filtering the bar construction}

The spectral sequence we want to construct comes from a filtration on the bar construction by the number of vertices in the underlying trees. In this section we construct this filtration and calculate the filtration quotients.

Definition 9.6 (Filtration on the category of trees) Write $\operatorname{Tree}_{s}(A)$ for the subcategory of Tree $(A)$ whose objects are the (isomorphism classes of) trees with less than or equal to $s$ (internal) vertices. We then have

$$
\operatorname{Tree}_{0}(A) \subset \operatorname{Tree}_{1}(A) \subset \cdots \subset \operatorname{Tree}_{|A|-1}(A)=\operatorname{Tree}(A) .
$$

Each $\operatorname{Tree}_{s}(A)$ is an initial subcategory of $\operatorname{Tree}(A)$. That is, if $U \leq T$ and $T \in \operatorname{Tree}_{s}(A)$ then $U \in \operatorname{Tree}_{s}(A)$. The filtration 'quotients' are the discrete categories

$$
\mathrm{Q}_{s}(A):=\operatorname{Tree}_{s}(A)-\operatorname{Tree}_{s-1}(A)
$$


whose objects are the trees with precisely $s$ vertices. For each tree $T \in \operatorname{Tree}(A)$ we write $|T|$ for the number of vertices of $T$.

Definition 9.7 (Filtration on the two-sided bar construction) For a reduced operad $P$ in $\mathcal{C}$ with right module $R$ and left module $L$, define

$$
B(R, P, L)_{s}(A):=\int^{T \in \operatorname{Tree}_{s}(A)} w(T)_{+} \otimes(R, P, L)_{A}(T) .
$$

For varying finite sets $A$ these form a symmetric sequence in $\mathcal{C}$. From the inclusion of categories $\operatorname{Tree}_{s-1}(A) \subset \operatorname{Tree}_{s}(A)$ we get natural maps

$$
B(R, P, L)_{s-1}(A) \rightarrow B(R, P, L)_{s}(A) .
$$

In the case $\mathcal{C}=\mathcal{T}$, it is easy to see that the resulting sequence of maps is a filtration of $B(R, P, L)(A)$ by subspaces. The subspace $B(R, P, L)_{s}(A)$ consists of those points in $B(R, P, L)(A)$ that can be represented by trees with less than or equal to $s$ vertices.

Example 9.8 The generalized $A$-labelled trees with no vertices (i.e. only a root and some leaves) correspond one-to-one with (unordered) partitions of $A$. We therefore see that

$$
B(R, P, L)_{0}=R \circ L
$$

where $\circ$ is the composition product of symmetric sequences.

Example 9.9 Take $R=L=I$ so that $B(R, P, L)=B(P)$. We then have $B(P)_{0}=I$ by the previous example. If $|A|>1$ there is precisely one (nongeneralized) $A$-labelled tree with only one vertex and we therefore get

$$
B(P)_{1}(A)= \begin{cases}S^{1} \otimes P(A) & \text { if }|A|>1 \\ B(P)(1) \cong S & \text { if }|A|=1\end{cases}
$$

where $S$ is the unit of the symmetric monoidal category $\mathcal{C}$.

We can think of the sequence

$$
B(R, P, L)_{0}(A) \rightarrow B(R, P, L)_{1}(A) \rightarrow \cdots \rightarrow B(R, P, L)(A)
$$

as a kind of 'cellular' filtration. That is, we obtain $B(R, P, L)_{s}(A)$ by attaching 'cells' to $B(R, P, L)_{s-1}(A)$, one for each generalized $A$-labelled tree $T$ with exactly $s$ vertices. The following proposition makes this precise. 
Proposition 9.10 There is a pushout square in $\mathcal{C}$ of the form

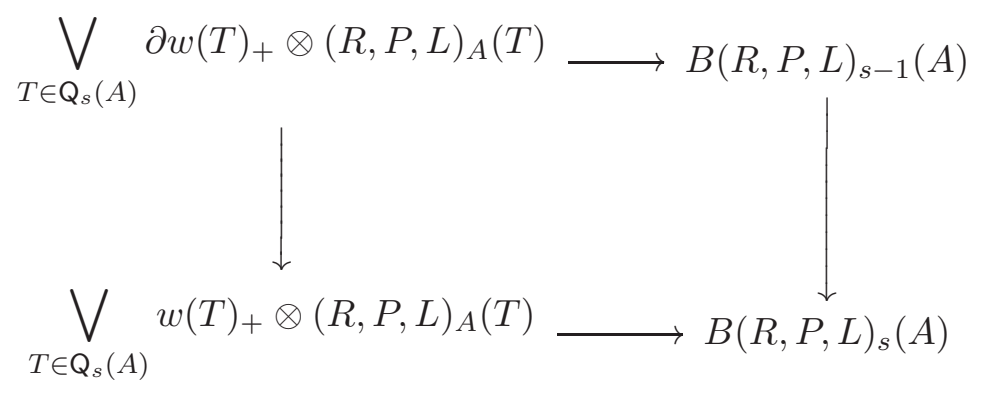

where $\partial w(T)$ denotes the boundary of the space $w(T)$.

To identify the top horizontal map in this diagram we use the following simple but important lemma.

Lemma 9.11 Let $T$ be a generalized $A$-labelled tree. Then

$$
\partial w(T)_{+} \cong \operatorname{colim}_{U<T} w(U)_{+} .
$$

The indexing category of the colimit is the full subcategory of $U \in \operatorname{Tree}(A)$ with $U<T$.

Proof of Lemma This is a categorical reflection of that fact (Lemma 7.5) that the boundary $\partial w(T)$ consists precisely of those weightings of $T$ in which some edge has length zero.

Proof of Proposition 9.10 The top horizontal map in the diagram is given by

$$
\begin{aligned}
\bigvee_{T \in Q_{s}(A)} \partial w(T)_{+} \otimes(R, P, L)_{A}(T) & \cong \bigvee_{T \in Q_{s}(A)} \underset{U<T}{\operatorname{colim}}\left[w(U)_{+} \otimes(R, P, L)_{A}(T)\right] \\
& \longrightarrow \bigvee_{T \in Q_{s}(A)} \underset{U<T}{\operatorname{colim}}\left[w(U)_{+} \otimes(R, P, L)_{A}(U)\right] \\
& \longrightarrow B(R, P, L)_{s-1}(A) .
\end{aligned}
$$

Here we've used the fact that $-\otimes C$ is a left adjoint so commutes with colimits. If $T \in Q_{s}(A)$ and $U<T$ then $U \in \operatorname{Tree}_{s-1}(A)$ so there are compatible maps from $w(U)_{+} \otimes(R, P, L)_{A}(U)$ to the coend defining $B(R, P, L)_{s-1}(A)$. 
With this definition, it is a simple exercise in naturality and colimits to see that the square commutes. To see that it is a pushout, take a commutative diagram

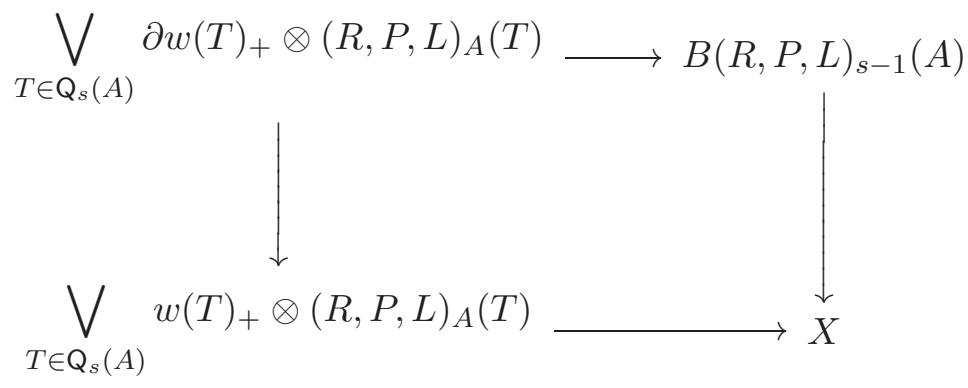

We have to show that this factors via a unique map

$$
B(R, P, L)_{s}(A) \rightarrow X .
$$

Since $B(R, P, L)_{s}(A)$ is a coend and hence a colimit, it is enough to get a unique set of compatible maps

$$
w(U)_{+} \otimes(R, P, L)_{A}(T) \rightarrow X
$$

for $U \leq T$ in $\operatorname{Tree}_{s}(A)$. If $T \notin \mathrm{Q}_{s}(A)$ the required map comes from the right-hand edge of diagram $(*)$. So suppose that $T \in \mathrm{Q}_{s}(A)$. Then we have

$$
w(U)_{+} \otimes(R, P, L)_{A}(T) \rightarrow w(T)_{+} \otimes(R, P, L)_{A}(T) \rightarrow X
$$

where the second map comes from the bottom edge of diagram $(*)$. We leave the reader to check that these maps are compatible in the appropriate way and suitably unique. We conclude that $B(R, P, L)_{s}(A)$ is the claimed pushout.

We use this result to identify the quotients of our filtration of the bar construction.

Corollary 9.12 Let $P$ be a reduced operad in $\mathcal{C}$ with right module $R$ and left module $L$. The following is a pushout square in $\mathcal{C}$ :

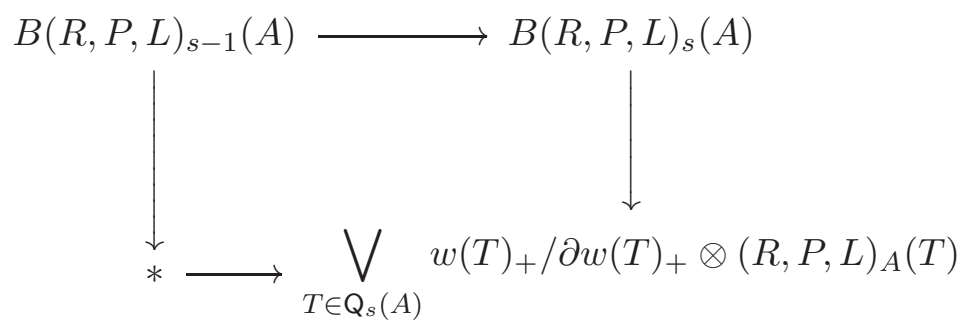


Proof Since $-\otimes C$ preserves colimits, the following is a pushout square in $\mathcal{C}$ :

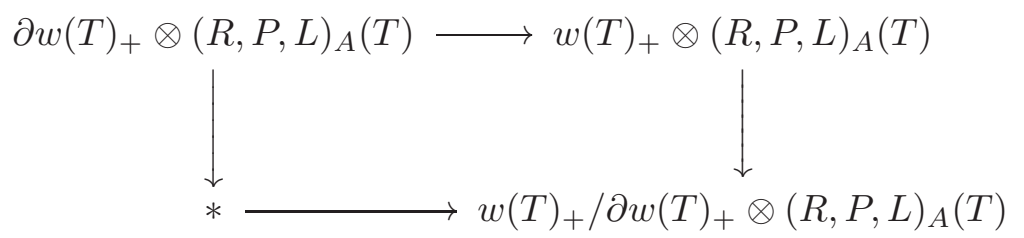

The corollary now follows from Proposition 9.10 and the universal properties of colimits.

Remark 9.13 Recall from Lemma 7.5 that for any generalized $A$-labelled tree $T$ with $s$ vertices, $w(T) \cong D^{s}$. Therefore, $w(T)_{+} / \partial w(T)_{+} \cong S^{s}$. We will be talking a lot about these spaces in the coming sections, so we will give them some more compact notation:

$$
\underline{w}(T):=w(T)_{+} / \partial w(T)_{+} \cong w(T) / \partial w(T) \cong S^{s}
$$

The results for the cobar construction are, as usual, just the duals of those for the bar construction. We summarize these briefly.

Definition 9.14 (Cofiltration of the cobar construction) Let $Q$ be a reduced cooperad in a symmetric monoidal $\mathcal{T}$-category $\mathcal{C}$ with right comodule $R$ and left comodule $L$. Then the two-sided cobar construction $\Omega(R, Q, L)$ has a 'cofiltration', that is, there is a sequence

$$
\Omega(R, Q, L)(A) \rightarrow \cdots \rightarrow \Omega(R, Q, L)^{s}(A) \rightarrow \Omega(R, Q, L)^{s-1}(A) \rightarrow \cdots
$$

where

$$
\Omega(R, Q, L)^{s}(A):=\int_{T \in \operatorname{Tree}_{s}(A)} \operatorname{Map}_{\mathcal{C}}\left(w(T)_{+},(R, Q, L)_{A}(T)\right),
$$

and the 'projection' map

$$
\Omega(R, Q, L)^{s}(A) \rightarrow \Omega(R, Q, L)^{s-1}(A)
$$

comes from the inclusion of categories $\operatorname{Tree}_{s-1}(A) \rightarrow \operatorname{Tree}_{s}(A)$ for $s \geq 1$.

Corollary 9.15 With $Q, R, L$ as in Definition 9.14 the following is a pullback 
square:

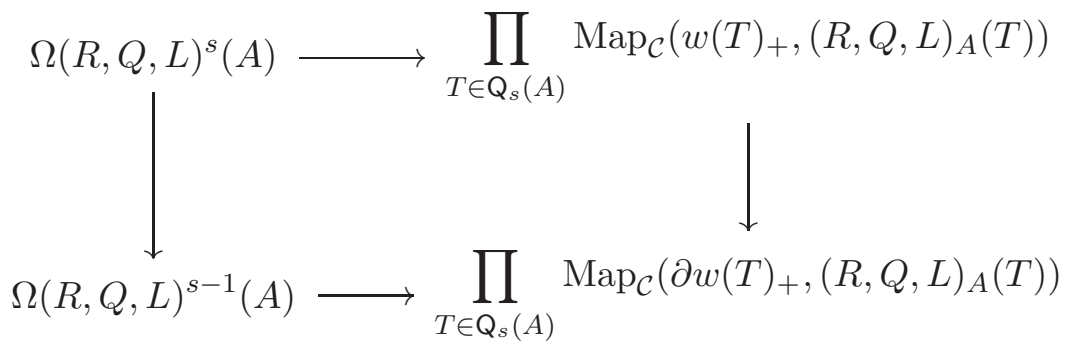

We can identify the fibres of the projections by the pullback squares

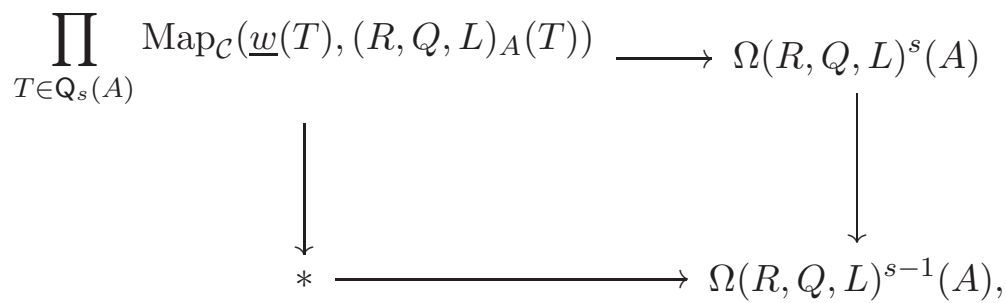

where $\underline{w}(T)=w(T) / \partial w(T)$.

\subsection{Conditions for the inclusion maps of the filtration to be cofibrations}

In the case that $\mathcal{C}$ is either $\mathcal{T}$ or $\mathcal{S} p$, the filtration of Section 9.2 allows us to construct a spectral sequence converging to the homology of $B(R, P, L)$. The $E^{1}$ term of this spectral sequence is given by the homologies of the homotopy cofibres of the inclusion maps of the filtration. In this section we give conditions under which these inclusions are cofibrations (in the standard model category structures on $\mathcal{T}$ and $\mathcal{S} p$ ) and which therefore ensure that the homotopy cofibres are given by the strict cofibres, or filtration quotients, that we have already calculated.

We state the main result of this section (Proposition 9.19 below) for a general symmetric monoidal $\mathcal{T}$-category $\mathcal{C}$ with a compatible model structure. Definition 9.16 says what we mean by 'compatible' here. We use Mark Hovey's book 13 as our basic reference for model categories.

Definition 9.16 A symmetric monoidal $\mathcal{T}$-model category is a symmetric monoidal $\mathcal{T}$-category $\mathcal{C}$ (as in Section 1 ) together with a model structure (in 
the sense of [13, Definition 1.1.3]) such that the tensoring makes $\mathcal{C}$ into a $\mathcal{T}$ model category (in the sense of [13, Definition 4.2.18]). That is, if $X \rightarrow Y$ is a cofibration in $\mathcal{T}$ and $C \rightarrow D$ is a cofibration in $\mathcal{C}$ then the induced map

$$
(X \otimes D) \amalg_{X \otimes C}(Y \otimes C) \rightarrow Y \otimes D
$$

is a cofibration in $\mathcal{C}$ that is trivial if either of our original cofibrations is. (The domain of this map is the pushout of $X \otimes D$ and $Y \otimes C$ over $X \otimes C$.)

Remark 9.17 We should say a few words about this definition. Firstly, we are not requiring that $\mathcal{C}$ be a monoidal model category in its own right (in the sense of [13, Section 4.2.6]). That is, we are not insisting that the symmetric monoidal structure $\bar{\wedge}$ on $\mathcal{C}$ in any way respect the model structure. Our reason for doing this is to preserve the self-duality of Definition 9.16 (see Lemma 9.21] below). In general, the opposite category of a monoidal model category is not another monoidal model category and we wish to dualize our theory to obtain results on the cobar construction.

On the other hand, the hypotheses we need to prove Proposition 9.19 are natural consequences of the assumption that $\mathcal{C}$ is a monoidal model category, and the categories we are most interested in, $\mathcal{T}$ and $\mathcal{S} p$, satisfy this assumption. This suggests that a breaking of the symmetry between bar and cobar is necessary when we come to study the homotopy theory of these constructions. In this paper, we do not pretend to give the beginnings of such a theory and, in particular, we do not claim that Definition 9.16 is the philosophically correct way to mix model category theory into this paper. For us, it serves the purposes of allowing us to make calculations with our spectral sequence in cases that are of interest.

Lemma 9.18 Let $\mathcal{C}$ be a symmetric monoidal $\mathcal{T}$-model category. If $C \in \mathcal{C}$ is cofibrant and $X \rightarrow Y$ is a cofibration in $\mathcal{T}$ then $X \otimes C \rightarrow Y \otimes C$ is a cofibration in $\mathcal{C}$.

Proof Apply the definition of $\mathcal{T}$-model category to the cofibrations $X \rightarrow Y$ and $* \rightarrow C$.

Proposition 9.19 Let $\mathcal{C}$ be a symmetric monoidal $\mathcal{T}$-model category such that if $C, D$ are cofibrant then $C \wedge D$ is also cofibrant. Let $P$ be a reduced operad in $\mathcal{C}$ with right module $R$ and left module $L$ such that, for all $A$, the objects $P(A), R(A), L(A)$ are cofibrant. Then, for all $s \geq 1$ and all finite sets $A$, the map

$$
B(R, P, L)_{s-1}(A) \rightarrow B(R, P, L)_{s}(A)
$$

of Definition 9.7 is a cofibration in $\mathcal{C}$. 
Proof The cofibrancy conditions on the $P(A), L(A), R(A)$ together with the extra condition on $\mathcal{C}$ ensure that the objects $(R, P, L)_{A}(T)$ are all cofibrant. For any generalized tree $T$, the map

$$
\partial w(T)_{+} \rightarrow w(T)_{+}
$$

is a cofibration in $\mathcal{T}$ (it is the inclusion of the boundary of a ball). Therefore, by Lemma 9.18 ,

$$
\partial w(T)_{+} \otimes(R, P, L)_{A}(T) \rightarrow w(T)_{+} \otimes(R, P, L)_{A}(T)
$$

is a cofibration. Proposition 9.10 tells us that the filtration map

$$
B(R, P, L)_{s-1}(A) \rightarrow B(R, P, L)_{s}(A)
$$

is a pushout of a coproduct of such maps so it too is a cofibration.

Remark 9.20 As we commented in Remark 9.17 above, if $\mathcal{C}$ is a symmetric monoidal model category in its own right, we get for free that $C$ and $D$ cofibrant imply $C \bar{\wedge} D$ cofibrant. In particular this is the case for $\mathcal{T}$ and $\mathcal{S} p$ (that is, the $S$-modules of EKMM [6] $)$.

As promised, our definition of symmetric monoidal $\mathcal{T}$-model category is selfdual.

Lemma 9.21 Let $\mathcal{C}$ be a symmetric monoidal $\mathcal{T}$-model category. Then $\mathcal{C}^{o p}$ is also a symmetric monoidal $\mathcal{T}$-model category with the standard dual symmetric monoidal and model structures.

Proof We already know from Proposition 1.12 that $\mathcal{C}^{\text {op }}$ is a symmetric monoidal $\mathcal{T}$-category. Recall that the tensoring for $\mathcal{C}^{\text {op }}$ is given by the cotensoring for $\mathcal{C}$, the cofibrations in $\mathcal{C}^{\text {op }}$ are the fibrations in $\mathcal{C}$ and a pushout in $\mathcal{C}^{\text {op }}$ is a pullback in $\mathcal{C}$. The weak equivalences in $\mathcal{C}^{\text {op }}$ are the same as those in $\mathcal{C}$.

To see that $\mathcal{C}^{\text {op }}$ is a $\mathcal{T}$-model category we have to show that if $X \rightarrow Y$ is a cofibration in $\mathcal{T}$ and $D \rightarrow C$ a fibration in $\mathcal{C}$ then

$$
\operatorname{Map}_{\mathcal{C}}(Y, D) \rightarrow \operatorname{Map}_{\mathcal{C}}(Y, C) \times_{\operatorname{Map}_{\mathcal{C}}(X, C)} \operatorname{Map}_{\mathcal{C}}(X, D)
$$

is a fibration in $\mathcal{C}^{\text {op }}$ that is trivial if either of our original maps is a weak equivalence. This result is given by Lemma 4.2.2 of [13].

The result dual to Proposition 9.19 is then the following. 
Corollary 9.22 Let $\mathcal{C}$ be a symmetric monoidal $\mathcal{T}$-model category such that if $C, D$ are fibrant then $C \wedge D$ is also fibrant. Let $Q$ be a reduced cooperad in $\mathcal{C}$ with right comodule $R$ and left comodule $L$ such that all the objects $Q(A), R(A), L(A)$ are fibrant. Then the map

$$
\Omega(R, Q, L)^{s}(A) \rightarrow \Omega(R, Q, L)^{s-1}(A)
$$

of Definition 9.14 is a fibration in $\mathcal{C}$.

In these circumstances, then, the fibres of the maps in the tower for $\Omega(R, Q, L)$ are also the homotopy fibres and so can be used to calculate the $E^{1}$ term of the associated spectral sequence.

Remark 9.23 In our categories of interest, $\mathcal{T}$ and $\mathcal{S} p$, all objects are fibrant and so the conditions of Corollary 9.22 hold for any cooperad and any comodules over it.

\subsection{The algebraic bar and cobar constructions}

So far we have constructed (under suitable conditions) a filtration of the twosided bar construction $B(R, P, L)$ by a sequence of cofibrations. This filtration yields a homology spectral sequence whose $E^{1}$ term turns out to be given by an algebraic version of our bar construction. In fact, it was this algebraic version, previously studied by Ginzburg-Kapranov [9], Getzler-Jones [8] and Fresse [7] among others, that inspired our definition of the bar construction for operads in topological settings. This section is devoted to the description of this algebraic bar construction. As in the topological case, we will only deal with reduced operads, that is, those for the unit map $k \rightarrow P(1)$ is an isomorphism.

Our definition of the algebraic bar construction emphasizes its similarity to the topological versions of Section 4 and Section 7 and it will follow the same pattern.

Definition 9.24 Let $P$ be a reduced operad in the category $\mathrm{Ch}_{k}$ of chain complexes over the commutative ring $k$ (with the symmetric monoidal structure of Definition 9.1). Let $R$ be a right $P$-module and $L$ a left $P$-module. More or less repeating Definition 7.7 we define a functor

$$
(R, P, L)_{A}: \operatorname{Tree}(A)^{\mathrm{op}} \rightarrow \mathrm{Ch}_{k}
$$

for each nonempty finite set $A$ by the formula

$$
(R, P, L)_{A}(T):=R(i(r)) \otimes \bigotimes_{\text {vertices } v \in T} P(i(v)) \otimes \bigotimes_{\text {leaves } l \in T} L\left(\iota^{-1} l\right) .
$$


The composition maps for $R, P$ and $L$ make $(R, P, L)_{A}$ into a functor as claimed. In making explicit calculations we have to be careful with the signs involved in the symmetry isomorphism for $\otimes$ but for theoretical purposes we can treat $(R, P, L)_{A}(T)$ as an unordered tensor product (see Remark [1.2).

We now wish to define the bar construction $B(R, P, L)$ by the same coend formula as in Definition 7.8 For this we need chain complex versions of the spaces $w(T)$ of weightings on trees $T \in \operatorname{Tree}(A)$. As in the topological case, the structures of these 'spaces', and how they fit together for different trees, are the key parts of the definition of the bar construction.

Definition 9.25 Let $T$ be a generalized $A$-labelled tree. The chain complex $C_{*} w(T)$ representing the space of weightings on $T$ will be the cellular chain complex for a certain cellular decomposition of the space $w(T)$. The cells in this decomposition correspond one-to-one with the trees $U \in \operatorname{Tree}(A)$ with $U \leq T$. The $r$-skeleton of $w(T)$ is given by

$$
\operatorname{sk}_{r} w(T):=\underset{U<T: U \in \operatorname{Tree}_{r}(A)}{\operatorname{colim}} w(U) .
$$

The attaching map for the cell corresponding to the tree $U$ with $r+1$ vertices is the map

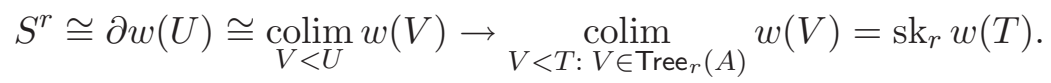

The cellular chain complex for this cell structure then has

$$
C_{r} w(T)=\bigoplus_{U \leq T: U \in \mathrm{Q}_{r}(A)} H_{r}(w(U), \partial w(U)) \cong \bigoplus_{U \leq T: U \in \mathrm{Q}_{r}(A)} \widetilde{H}_{r}(\underline{w}(U)) .
$$

Recall from Remark 9.13 that $\underline{w}(U)$ denotes the quotient $w(U) / \partial w(U)$. The differential

$$
C_{r} w(T) \rightarrow C_{r-1} w(T)
$$

is given by summing the map 13

$$
\widetilde{H}_{r}(\underline{w}(U)) \cong H_{r}(w(U), \partial w(U)) \rightarrow \widetilde{H}_{r-1}\left(\partial w(U)_{+}\right) \rightarrow \widetilde{H}_{r-1}(\underline{w}(V))
$$

\footnotetext{
${ }^{13}$ The last part of this composite comes from the map

$$
\partial w(U)_{+} \cong \underset{V<U}{\operatorname{colim}} w(V)_{+} \rightarrow w(V)_{+} / \partial w(V)_{+}=\underline{w}(V)
$$

given by collapsing to the basepoint everything except the interior of the 'face' $w(V)$ of $\partial w(U)$.
} 
for pairs $(U, V)$ with $V<U,|U|=r$ and $|V|=r-1$. An example of this chain complex for a particular tree is shown in Figure 15.

The inclusion $w(U) \rightarrow w(T)$ is cellular and so we have inclusions

$$
C_{*} w(U) \rightarrow C_{*} w(T)
$$

for $U<T$. These make $C_{*} w(-)$ into a functor

$$
C_{*} w(-): \operatorname{Tree}(A) \rightarrow \mathrm{Ch}_{k} .
$$

This is the chain complex analogue of the functor $w(-)$ of Definition [7.6.

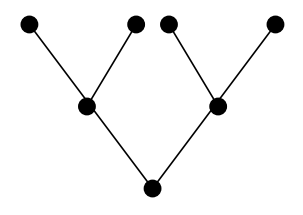

$T$

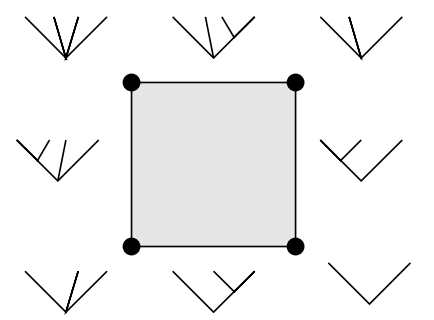

$w(T)$

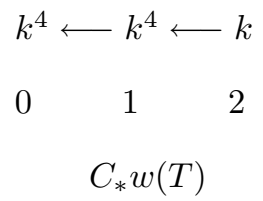

$C_{*} w(T)$

Figure 15: Example of the chain complex $C_{*} w(T)$ showing the cellular decomposition of $w(T)$

Definition 9.26 With our 'chain complexes of weighted trees' $C_{*} w(T)$, we now define the two-sided algebraic bar construction on the reduced operad $P$ with coefficients in $R$ and $L$ to be the symmetric sequence $B(R, P, L)$ with

$$
B(R, P, L)(A):=\int^{T \in \operatorname{Tree}(A)} C_{*} w(T) \otimes(R, P, L)_{A}(T) .
$$

This coend is calculated in the category of chain complexes on $k$ and results in a chain complex $B(R, P, L)(A)$. However, it will be useful to consider a bicomplex structure on $B(R, P, L)(A)$ for which this chain complex is the total complex. The bicomplex structure comes about by considering the tensor product of the chain complexes $C_{*} w(T)$ and $(R, P, L)_{A}(T)$ as a bicomplex with gradings and differentials coming from these separate terms. We will write

$$
B(R, P, L)_{*, *}(A)
$$

to emphasize this bigrading with the first index denoting the grading that comes from $C_{*} w(T)$ (we'll call this the tree grading) and the second the grading that 
comes from $(R, P, L)_{A}(T)$ (which we'll call the internal grading). We then have two separate differentials on $B(R, P, L)_{*, *}$ :

$$
\partial: B(R, P, L)_{*, *} \rightarrow B(R, P, L)_{*-1, *}
$$

coming from the differentials on the chain complexes $C_{*} w(T)$ which will refer to as the tree differential on the bar construction, and

$$
d: B(R, P, L)_{*, *} \rightarrow B(R, P, L)_{*, *-1}
$$

coming from the differentials on the $(R, P, L)_{A}(T)$ which we will call the internal differential.

In later sections, we will be applying the algebraic bar construction to operads of graded $k$-modules, that is, chain complexes with zero differential. In this case, the internal differential of $B(R, P, L)(A)$ will be zero.

We can give a more explicit description of $B(R, P, L)$ as follows.

Lemma 9.27 Let $P$ be a reduced operad in $\mathrm{Ch}_{k}$ with right module $R$ and left module $L$. Then we hav 14

$$
B(R, P, L)_{s, *}(A) \cong \bigoplus_{T \in Q_{s}(A)} \widetilde{H}_{s}(\underline{w}(T)) \otimes(R, P, L)_{A}(T)
$$

as chain complexes of $k$-modules with respect to the internal grading and differential.

Under these isomorphisms, the tree differential

$$
\partial: B(R, P, L)_{s, *}(A) \rightarrow B(R, P, L)_{s-1, *}(A)
$$

is given by summing, over all pairs $(T, U)$ with $U<T,|T|=s$ and $|U|=s-1$, the maps

$$
\widetilde{H}_{s}(\underline{w}(T)) \otimes(R, P, L)_{A}(T) \rightarrow \widetilde{H}_{s-1}(\underline{w}(U)) \otimes(R, P, L)_{A}(U)
$$

obtained by combining the maps

$$
(R, P, L)_{A}(T) \rightarrow(R, P, L)_{A}(U)
$$

with the terms

$$
\widetilde{H}_{s}(\underline{w}(T)) \rightarrow \widetilde{H}_{s-1}(\underline{w}(U))
$$

from the top differential of the chain complex $C_{*} w(T)$.

\footnotetext{
${ }^{14}$ Here, as elsewhere, the reduced homology of the quotient $\underline{w}(T)=w(T) / \partial w(T)$ can be replaced with the homology of the pair $(w(T), \partial w(T))$. Both of these are isomorphic to the graded module $k$ concentrated in degree $|T|$.
} 
Proof We consider a filtration of the algebraic bar construction analogous to that of Section 9.2 for the topological version. Virtually the same analysis applies and we get short exact sequences of chain complexe 15

$$
B(R, P, L)_{s-1}(A) \rightarrow B(R, P, L)_{s}(A) \rightarrow \bigoplus_{T \in Q_{s}(A)} C_{*} w / C_{*} \partial w(T) \otimes(R, P, L)_{T}(A) .
$$

where $C_{*} \partial w(T)$ is the cellular chain complex for the subcomplex $\partial w(T) \subset w(T)$ (that is, everything except the top-dimension cell). Notice that

$$
C_{*} w(T) / C_{*} \partial w(T) \cong \widetilde{H}_{s}(\underline{w}(T))
$$

for $T \in \mathrm{Q}_{s}(A)$. We construct a splitting of the above short exact sequence (with respect to the internal differential but not the tree differential) using the obvious splittings (as $k$-modules) of the sequences

$$
C_{*} \partial w(T) \rightarrow C_{*} w(T) \rightarrow \widetilde{H}_{*}(\underline{w}(T)) .
$$

We get by induction on $s$ that

$$
B(R, P, L)(A) \cong \bigoplus_{T \in \operatorname{Tree}(A)} \widetilde{H}_{|T|}(\underline{w}(T)) \otimes(R, P, L)_{A}(T)
$$

which splits, by tree degree, into the isomorphisms of the lemma. We leave the reader to check that the tree differential has the promised formula.

Remark 9.28 Choosing generators of the groups $H_{|T|}(w(T), \partial w(T)) \cong k$ determines an isomorphism

$$
B(R, P, L)(A) \cong \bigoplus_{T \in \operatorname{Tree}(A)}(R, P, L)_{A}(T)
$$

which is the definition of the algebraic bar construction given by Fresse in [7, Section 4.4]. Such choices determine choices of the coefficients (in fact, signs) for the maps that make up the differential $\partial$ on $B(R, P, L)(A)$.

Fresse shows that this bar construction is a representative of the derived composition product of $R$ and $L$ as $P$-modules, that is,

$$
B(R, P, L) \simeq R \circ_{P}^{\mathbb{L}} L
$$

\footnotetext{
${ }^{15}$ The notation here is probably rather confusing. We are using $B(R, P, L){ }_{s}(A)$ to denote the part of the filtration of $B(R, P, L)(A)$ obtained via the chain complex version of Definition 9.7 This is not to be confused with $B(R, P, L)_{s, *}$ which is the graded summand of tree degree $s$. In fact, it's a consequence of the proof of this lemma that

$$
B(R, P, L)_{s}(A) \cong \bigoplus_{r \leq s} B(R, P, L)_{r, *}(A) .
$$
}


and so the homology groups of $B(R, P, L)$, with respect to the tree differential, are Tor groups of $P$-modules.

The relationship between this algebraic bar construction and the simplicial bar construction was analyzed by Fresse. His proof of the following proposition uses a 'levelization' process analogous to that we used in the proof of Proposition 4.13

Proposition 9.29 ([7, Theorem 4.1.8]) The algebraic two-sided bar construction $B(R, P, L)$ is quasi-isomorphic to the normalized chain complex of the simplicial bar construction on $P$ with coefficients in $R$ and $L$ (the algebraic version of Definition [7.9).

As usual, we have the dual constructions and results.

Definition 9.30 Let $Q$ be a reduced cooperad of chain complexes of $k-$ modules with right comodule $R$ and left comodule $L$. Then there are functors $(R, Q, L)_{A}$ from $\operatorname{Tree}(A)$ to $\mathrm{Ch}_{k}$ and we can define the algebraic cobar construction on $Q$ with coefficients in $R$ and $L$ by the same formula

$$
\Omega(R, Q, L)(A):=\int_{T \in \operatorname{Tree}(A)} \operatorname{Hom}\left(C_{*} w(T),(R, Q, L)_{A}(T)\right)
$$

as in Definition [5.1] where, for chain complexes $M, N$, $\operatorname{Hom}(M, N)$ denotes the chain complex of maps of graded modules $M \rightarrow N$. The cobar construction is a bicomplex with an internal grading and differential coming from the $(R, Q, L)_{A}(T)$ and a tree grading and differential $\partial^{*}$ coming from the $C_{*} w(T)$. We follow the convention that $\operatorname{Hom}(M, N)_{s, t}=\operatorname{Hom}\left(M_{-s}, N_{t}\right)$ so that the tree grading on the cobar construction is concentrated in negative degrees.

There is an explicit description of the cobar construction analogous to that of Lemma 9.27 for the bar construction.

Lemma 9.31 With $Q, R, L$ as above:

$$
\Omega(R, Q, L)_{-s, *}(A):=\bigoplus_{T \in Q_{s}(A)} \operatorname{Hom}\left(\widetilde{H}_{s}(\underline{w}(T)),(R, Q, L)_{A}(T)\right)
$$

which again is just isomorphic to

$$
\bigoplus_{T \in \operatorname{Tree}(A)}(R, Q, L)_{A}(T)
$$


after choosing generators of the groups $\widetilde{H}_{s}(\underline{w}(T))$. The internal grading and differential correspond in the obvious way under this isomorphism. The explicit form of the tree differential $\partial^{*}$ is given by the maps

$$
(R, Q, L)_{A}(U) \rightarrow(R, Q, L)_{A}(T)
$$

with coefficients again given by the components

$$
\widetilde{H}_{s}(\underline{w}(T)) \rightarrow \widetilde{H}_{s-1}(\underline{w}(U))
$$

of the top differential on the chain complex $C_{*} w(T)$.

Definition 9.32 When $P$ is a reduced operad in the category of graded $k-$ modules, the unit symmetric sequence $I$ defined by

$$
I(A):= \begin{cases}k & \text { if }|A|=1 ; \\ 0 & \text { otherwise. }\end{cases}
$$

is both a left and right $P$-module. The reduced bar construction on $P$ is then given by the two-sided bar construction with coefficients in $I$ on both sides:

$$
B(P):=B(I, P, I)
$$

The definition of the algebraic bar construction reduces in this case to

$$
B(P)(A)=\int^{T \in \mathrm{T}(A)} C_{*} \bar{w}(T) \otimes P_{A}(T) .
$$

Recall that the space $\bar{w}(T)$ is the quotient of $w(T)$ by the subspace $w_{0}(T)$ of weightings that give length 0 to either the root edge or a leaf edge of $T$. This subspace is in fact a subcomplex with respect to our chosen cellular structure on $w(T) 16$ Therefore we obtain a cell structure on $\bar{w}(T)$ and in the above formula, $C_{*} \bar{w}(T)$ denotes the relative cellular chain complex for the pair $(\bar{w}(T), *)$, or equivalently, for the pair $\left(w(T), w_{0}(T)\right) 17$ It is clear that $C_{*} \bar{w}(T)$ is a quotient of $C_{*} w(T)$.

It's also easy to check that by Lemma 9.27 we have

$$
B(P)(A) \cong \bigoplus_{T \in \mathbf{T}(A)} \widetilde{H}_{|T|}(\underline{w}(T)) \otimes P_{A}(T)
$$

\footnotetext{
${ }^{16}$ It is the union of the cells corresponding to $U<T$ that are not in the original category $\mathrm{T}(A)$, that is, that are generalized trees, but not trees in the sense of Section 3

${ }^{17}$ The tensor product of chain complexes is here playing the role of the smash product of based spaces so we need the reduced version of the cellular chain complex. Strictly speaking, the chain complex $C_{*} w(T)$ is the relative chain complex of the pair $\left(w(T)_{+}, *\right)$.
} 
which (after choosing isomorphisms $\widetilde{H}_{|T|}(\underline{w}(T)) \cong k$ ) is the original definition of the algebraic bar construction given in Getzler-Jones [8, Section 2.1].

Similarly, if $Q$ is a reduced cooperad then $I$ is both a left and right $Q$-comodule and the reduced cobar construction on $Q$ is

$$
\Omega(Q):=\Omega(I, Q, I)
$$

and is given by a formula analogous to that of Definition 5.1

As in the topological case, the reduced algebraic bar construction on a reduced operad $P$ of chain complexes has a cooperad structure. We now describe this.

Definition 9.33 The required maps

$$
B(P)\left(A \cup_{a} B\right) \rightarrow B(P)(A) \otimes B(P)(B)
$$

are defined in exactly the same way as the corresponding maps in the topological case (Definition 4.3). To do this we must construct the algebraic versions of the key maps (4.18):

$$
C_{*} \bar{w}\left(T \cup_{a} U\right) \rightarrow C_{*} \bar{w}(T) \otimes C_{*} \bar{w}(U)
$$

for $A$-labelled trees $T$ and $B$-labelled trees $U$. We get this by taking the map of cellular chain complexes induced by the topological map

$$
\bar{w}\left(T \cup_{a} U\right) \rightarrow \bar{w}(T) \wedge \bar{w}(U)
$$

of Definition 4.16] For this to work, we need the following lemma.

Lemma 9.34 Let $T$ be an $A$-labelled tree, $U$ a $B$-labelled tree and let $a \in A$. The map

$$
\bar{w}\left(T \cup_{a} U\right) \rightarrow \bar{w}(T) \wedge \bar{w}(U)
$$

is cellular, that is, it preserves skeleta.

Proof A point $p$ in $\bar{w}\left(T \cup_{a} U\right)$ is in the $s$-skeleton if and only if it is in the subspace $\bar{w}(V)$ for some tree $V$ with $s$ vertices. If this tree $V$ is not of type $(A, B)$ then $p$ is mapped to the basepoint which is certainly in the $s^{-}$ skeleton of the right-hand side. If $V$ is of type $(A, B)$ (that is, obtained by grafting an $A$-labelled tree $T^{\prime}$ and a $B$-labelled tree $\left.U^{\prime}\right)$ then the point $p$ maps to a pair consisting of a point in some $\bar{w}\left(T^{\prime}\right) \subset \bar{w}(T)$ and a point in some $\bar{w}\left(U^{\prime}\right) \subset \bar{w}(U)$. The first point is in the $s^{\prime}$-skeleton of $\bar{w}(T)$ where $T^{\prime}$ has $s^{\prime}$ vertices. The second point is in the $s^{\prime \prime}$-skeleton of $\bar{w}(U)$ where $U^{\prime}$ has $s^{\prime \prime}$ vertices. Therefore the pair is in the $s^{\prime}+s^{\prime \prime}$-skeleton of $\bar{w}(T) \wedge \bar{w}(U)$. However, since $V$ only had $s$ vertices, we must have $s^{\prime}+s^{\prime \prime} \leq s$. So the image of $p$ is in the $s$-skeleton of $\bar{w}(T) \wedge \bar{w}(U)$ as required. 
It is easy to describe explicitly the resulting map of chain complexes

$$
C_{*} \bar{w}\left(T \cup_{a} U\right) \rightarrow C_{*} \bar{w}(T) \otimes C_{*} \bar{w}(U) .
$$

Recall that the left-hand side is given by the direct sum over $V \in \operatorname{Tree}\left(A \cup_{a} B\right)$ with $V \leq T \cup_{a} U$ of the homology groups $\widetilde{H}_{*}(\underline{w}(V))$. The above map sends the term corresponding to a tree $V$ that is not of type $(A, B)$, to zero. If $V=T^{\prime} \cup_{a} U^{\prime}$, then $T^{\prime} \leq T$ and $U^{\prime} \leq U$ and the corresponding term maps to the right-hand side via the isomorphism

$$
\widetilde{H}_{*}\left(\underline{w}\left(T^{\prime} \cup_{a} U^{\prime}\right)\right) \rightarrow \widetilde{H}_{*}\left(\underline{w}\left(T^{\prime}\right)\right) \otimes \widetilde{H}_{*}\left(\underline{w}\left(U^{\prime}\right)\right),
$$

which is induced by the homeomorphism 18

$$
\underline{w}\left(T^{\prime} \cup_{a} U^{\prime}\right) \rightarrow \underline{w}\left(T^{\prime}\right) \wedge \underline{w}\left(U^{\prime}\right),
$$

which in turn is a quotient of the map

$$
\bar{w}\left(T^{\prime} \cup_{a} U^{\prime}\right) \rightarrow \bar{w}\left(T^{\prime}\right) \wedge \bar{w}\left(U^{\prime}\right) .
$$

With this key map in place, the rest of the formal definition of the cooperad structure maps for the topological bar construction (Definition 4.26) carries over to the algebraic case.

Lemma 9.35 Let $P$ be a reduced operad in $\mathrm{Ch}_{k}$. Under the isomorphism of Lemma 9.27 the cooperad structure on $B(P)$ corresponds to the cooperad structure on the chain complexes

$$
\bigoplus_{T \in \mathrm{T}(A)} \widetilde{H}_{|T|}(\underline{w}(T)) \otimes P_{A}(T)
$$

whose cocomposition maps are given by summing over the maps obtained by combining the isomorphisms

$$
H_{*}\left(\underline{w}\left(T \cup_{a} U\right)\right) \rightarrow H_{*}(\underline{w}(T)) \otimes H_{*}(\underline{w}(U))
$$

with the isomorphisms

$$
P_{A \cup_{a} B}\left(T \cup_{a} U\right) \rightarrow P_{A}(T) \otimes P_{B}(U) .
$$

Proof This is a simple check using the definition of the isomorphism in Lemma 9.27 by splittings of short exact sequences.

\footnotetext{
${ }^{18}$ It is easy to check that this map is a bijection. The spaces involved are all spheres which are compact Hausdorff, so it is a homeomorphism.
} 
Remark 9.36 Choosing generators for the groups $\widetilde{H}_{*}(\underline{w}(T))$, we see that this is equivalent to the cooperad structure defined by Getzler-Jones $[\underline{8}$ and by Fresse [7].

Definition 9.37 Dually, if $Q$ is a reduced cooperad of chain complexes, there is an operad structure on the reduced algebraic cobar construction $\Omega(Q)$. The corresponding operad structure under the isomorphism of Lemma 9.31 is built from the isomorphisms

$$
Q_{A}(T) \otimes Q_{B}(U) \rightarrow Q_{A \cup_{a} B}\left(T \cup_{a} U\right)
$$

and the same maps

$$
\widetilde{H}_{*}\left(\underline{w}\left(T \cup_{a} U\right)\right) \rightarrow \widetilde{H}_{*}(\underline{w}(T)) \otimes \widetilde{H}_{*}(\underline{w}(U)) .
$$

Remark 9.38 It does not take much more effort to extend the cooperad and operad structure above to maps

$$
B(R, P, L) \rightarrow B(R, P, I) \widehat{\circ} B(I, P, L)
$$

and

$$
\Omega(R, Q, I) \circ \Omega(I, Q, L) \rightarrow \Omega(R, Q, L)
$$

following the same sort of generalization that we did in Section 7.3

\subsection{A spectral sequence for the homology of the bar construc- tion}

We now turn our attention directly to the homology spectral sequences born from the filtration of the bar construction and cofiltration of the cobar construction 19 The work we have done in the last few sections allows us to identify the $E^{1}$ terms of these spectral sequences, under suitable conditions, with the algebraic bar and cobar constructions.

A quick word on notation: from now on, the only topological categories $\mathcal{C}$ we are interested in are $\mathcal{T}$ and $\mathcal{S} p$. We will therefore drop the notation $\bar{\wedge}$ for the monoidal product and $\otimes$ for the tensoring over $\mathcal{T}$, replacing both with the standard notation $\wedge$. We will reserve $\otimes$ for the tensor product of graded $k$-modules.

\footnotetext{
${ }^{19}$ If $\mathcal{C}$ is the category of based spaces, we only get a spectral sequence for the bar construction and not for the cobar construction. This is because a fibre sequence in $\mathcal{T}$ does not immediately yield a long exact sequence in homology.
} 
Proposition 9.39 Let $P$ be a reduced operad in $\mathcal{T}$ or $\mathcal{S} p$ with right module $R$ and left module $L$ such that all the objects $P(A), R(A), L(A)$ are cofibrant and all homology groups $H_{*} P, H_{*} R, H_{*} L$ flat $k$-modules. Then for each finite set $A$ there is a spectral sequence converging to $H_{*} B(R, P, L)(A)$ with $E^{1}$-term and first differential given by the algebraic bar construction:

$$
\left(E^{1}, d^{1}\right) \cong\left(B\left(H_{*} R, H_{*} P, H_{*} L\right)(A), \partial\right) \Longrightarrow H_{*} B(R, P, L)(A) .
$$

Let $Q$ be a reduced cooperad in $\mathcal{S} p$ with right comodule $R$ and left comodule $L$ such that all the objects $Q(A), R(A), L(A)$ are fibran 20 and all the homology groups $H_{*} Q, H_{*} R, H_{*} L$ are flat $k$-modules. Then for each finite set $A$ there is a spectral sequence converging to $H_{*}(\Omega(R, Q, L)(A))$ with $E^{1}$-term and first differential given by the algebraic cobar construction:

$$
\left(E^{1}, d^{1}\right) \cong\left(\Omega\left(H_{*} R, H_{*} Q, H_{*} L\right)(A), \partial^{*}\right) \Longrightarrow H_{*} \Omega(R, Q, L)(A) .
$$

Remark 9.40 By the comments of Remark 9.28, the work of Fresse allows us to identify the $E^{2}$ terms of these spectral sequences as suitable Tor groups. That is, our spectral sequence take the form

$$
E^{2}=\operatorname{Tor}^{H_{*} P}\left(H_{*} R, H_{*} L\right) \Longrightarrow H_{*} B(R, P, L)
$$

and

$$
E^{2}=\operatorname{Tor}^{H_{*} Q}\left(H_{*} R, H_{*} L\right) \Longrightarrow H_{*} \Omega(R, Q, L) .
$$

This suggests that the topological bar and cobar constructions should have an interpretation as topological Tor objects. We have not yet studied the homotopy theory of these constructions sufficiently to make this precise.

Proof of Proposition 9.39 By Proposition 9.19 we have cofibre sequences

$$
B(R, P, L)_{s-1}(A) \rightarrow B(R, P, L)_{s}(A) \rightarrow \bigvee_{T \in Q_{s}(A)} \underline{w}(T) \wedge(R, P, L)_{A}(T)
$$

Summing these over $s$ we obtain an exact couple and hence a spectral sequence.

\footnotetext{
${ }^{20}$ This is really automatic since we have chosen $\mathcal{S} p$ to be the category of $S$-modules of EKMM [6] in which all objects are fibrant. If we want to work with other categories of spectra, however, we need this condition.
} 
The $E^{1}$ term of this spectral sequence is

$$
\begin{aligned}
E_{s, t}^{1} & :=H_{s+t}\left(\bigvee_{T \in Q_{s}(A)} \underline{w}(T) \wedge(R, P, L)_{A}(T)\right) \\
& \cong \bigoplus_{T \in Q_{s}(A)} H_{s+t}\left(\underline{w}(T) \wedge(R, P, L)_{A}(T)\right) \\
& \cong \bigoplus_{T \in Q_{s}(A)} \widetilde{H}_{s}(\underline{w}(T)) \otimes H_{t}\left((R, P, L)_{A}(T)\right) \\
& \cong \bigoplus_{T \in Q_{s}(A)} \widetilde{H}_{s}(\underline{w}(T)) \otimes\left(H_{*} R, H_{*} P, H_{*} L\right)_{A}(T)_{t} \\
& \cong B\left(H_{*} R, H_{*} P, H_{*} L\right)_{s, t}(A) .
\end{aligned}
$$

where we have made plentiful use of the Künneth formula. In particular, we need the flatness assumptions to get

$$
H_{*}\left((R, P, L)_{A}(T)\right) \cong\left(H_{*} R, H_{*} P, H_{*} L\right)_{A}(T) .
$$

The final isomorphism is that of Lemma 9.27 Since the filtration of each individual $B(R, P, L)(A)$ is finite, this spectral sequence certainly converges to $H_{*} B(R, P, L)(A)$. It remains to be shown that $d^{1}$ is given by the differential $\partial$ of the algebraic bar construction.

The differential $d^{1}$ is the composite

$$
\begin{aligned}
H_{*}\left(\bigvee_{T \in Q_{s}(A)} \underline{w}(T) \wedge(R, P, L)_{A}(T)\right) & \rightarrow H_{*-1} B(R, P, L)_{s-1}(A) \\
& \rightarrow H_{*-1}\left(\bigvee_{U \in Q_{s-1}(A)} \underline{w}(U) \wedge(R, P, L)_{A}(U)\right)
\end{aligned}
$$

of the boundary map in the long exact sequence associated to one of the cofibre sequences $(*)$, with the projection map from another one. To analyze this, fix for the moment a generalized $A$-labelled tree $T$ with $s$ vertices and consider the following map of cofibre sequences:

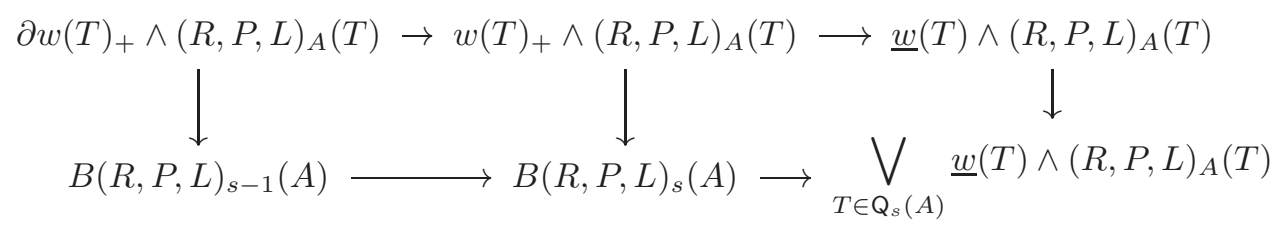


This induces a map of long exact sequences in homology, and in particular we have a commutative diagram

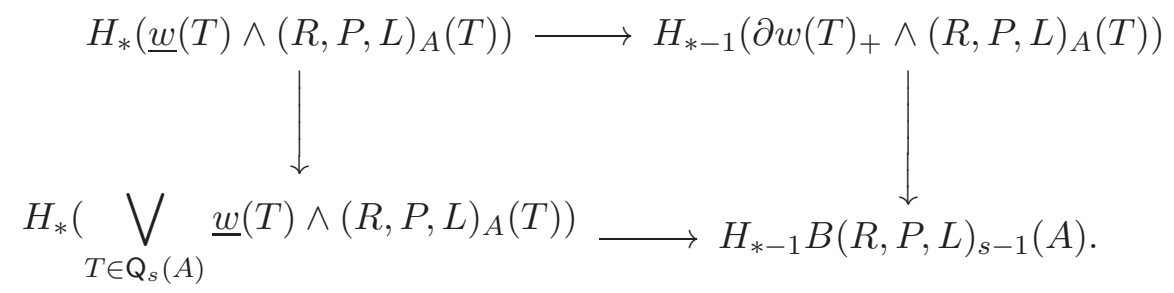

On the other hand, using the identity

$$
\partial w(T)_{+} \cong \operatorname{colim}_{U<T} w(U)_{+}
$$

we also have a commutative diagram

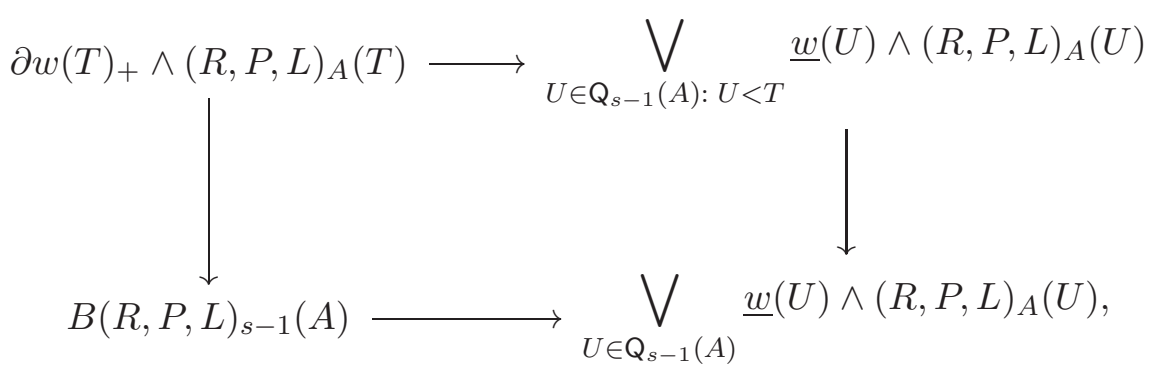

where the top horizontal map is constructed from the quotient maps

$$
\partial w(T)_{+} \rightarrow \underline{w}(U)
$$

for $U \in \operatorname{Tree}_{s-1}(A)$ such that $U<T$, together with the operad composition maps

$$
(R, P, L)_{A}(T) \rightarrow(R, P, L)_{A}(U) .
$$

Taking the homology of this diagram, combining it with our other diagram of homology groups, throwing in the Künneth formula, summing the top lines over all $T \in \mathrm{Q}_{s}(A)$ and using Lemma 9.27, we get the big commutative diagram of Figure 16] in which the top row is the differential $\partial$ on the algebraic bar construction $B\left(H_{*} R, H_{*} P, H_{*} L\right)(A)$ (under the isomorphism of Lemma 9.27) and the bottom row is the differential $d^{1}$ of our spectral sequence. The left and right sides of the diagram are the isomorphisms described at the beginning of this proof that identify $E^{1}$ with the algebraic bar construction. 


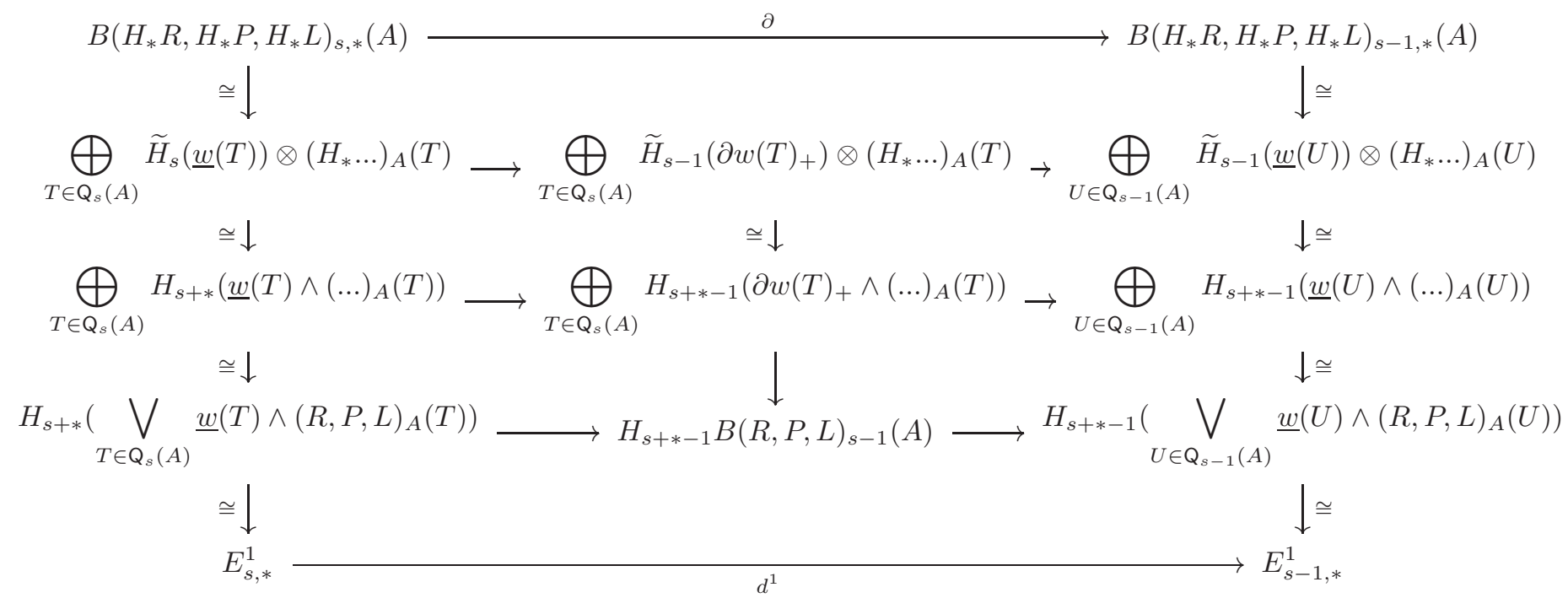

Figure 16: Another big commutative diagram. This shows that the tree differential on $B\left(H_{*} R, H_{*} P, H_{*} L\right)$ is the same as the $d^{1}$ differential in the spectral sequence of Proposition 9.39 Here $P$ is a reduced operad in $\mathcal{T}$ or $\mathcal{S} p$ with right module $R$ and left module $L$. In the second row, $\left(H_{*} \ldots\right)$ stands for $\left(H_{*} R, H_{*} P, H_{*} L\right)$ and in the middle row $(\ldots)$ stands for $(R, P, L)$. 
The argument for the cobar construction is dual but only applies when we are working in a category of spectra. The sequence of isomorphisms that identifies the $E^{1}$ term then takes the form

$$
\begin{aligned}
E_{-s, t}^{1} & :=H_{-s+t}\left(\prod_{T \in Q_{s}(A)} \operatorname{Map}_{\mathcal{S} p}\left(\underline{w}(T),(R, Q, L)_{A}(T)\right)\right) \\
& \left.\cong \bigoplus_{T \in Q_{s}(A)} H_{-s+t} \operatorname{Map}_{\mathcal{S} p}\left(\underline{w}(T),(R, Q, L)_{A}(T)\right)\right) \\
& \cong \bigoplus_{T \in Q_{s}(A)} \operatorname{Hom}\left(H_{s}(\underline{w}(T)), H_{t}(R, Q, L)_{A}(T)\right) \\
& \cong \bigoplus_{T \in Q_{s}(A)} \operatorname{Hom}\left(H_{s}(\underline{w}(T)),\left(H_{*} R, H_{*} Q, H_{*} L\right)_{A}(T)_{t}\right) \\
& \cong \Omega\left(H_{*} R, H_{*} Q, H_{*} L\right)_{s, t}(A) .
\end{aligned}
$$

In particular we use the fact that we are working with spectra and not based spaces to get the isomorphism

$$
H_{-s+t} \operatorname{Map}(\underline{w}(T), X) \cong H_{-s+t}\left(\Sigma^{-s} X\right) \cong H_{t} X \cong \operatorname{Hom}\left(H_{s}(\underline{w}(T)), H_{t} X\right)
$$

that replaces an application of the Künneth formula in the bar construction case.

Remark 9.41 Notice that the spectral sequence for the bar construction lies in the right half-plane (and the first quadrant if the objects $R(A), P(A), L(A)$ only have non-negative homology). That for the cobar construction lies in the left half-plane (and the second quadrant if the objects $R(A), Q(A), L(A)$ only have non-negative homology).

\subsection{The link to Koszul duality}

We now use our spectral sequence to look at the relationship between the bar construction on an operad in based spaces or spectra and Koszul duality. Koszul duality for operads initially appeared in Ginzburg-Kapranov [9]. Further references include Getzler-Jones [8] and Fresse [7].

The main result of this section is that if $P$ is a reduced operad in based spaces or spectra such that $H_{*} P$ is a Koszul operad in graded $k$-modules, then the spectral sequence for calculating $H_{*} B(P)$ collapses at the $E^{2}$-term and we conclude that $H_{*} B(P)$ is the Koszul dual cooperad of $H_{*} P$. This result is a simple consequence of the definitions of a Koszul operad and its Koszul dual cooperad. The dual result holds for cooperads in spectra. 
Definition 9.42 (Koszul operads) Let $P$ be a reduced operad in the category $\operatorname{Mod}_{k}$ of graded $k$-modules. We say $P$ is Koszul if the homology of the reduced bar construction on $P$ is concentrated in the top tree degree. We explain exactly what we mean by this. The reduced bar construction $B(P)$ is given by

$$
B(P)_{s, *}(A) \cong \bigoplus_{T \in \bar{Q}_{s}(A)} \widetilde{H}_{s}(\underline{w}(T)) \otimes P_{A}(T) .
$$

where $\overline{\mathrm{Q}}_{s}(A)=\mathrm{Q}_{s}(A) \cap \mathrm{T}_{s}(A)$ is the set of $A$-labelled trees (in the sense of Section 3 that is, not generalized trees) with exactly $s$ vertices. If $|A|=1$, this is concentrated in the column $s=0$. If $|A|>1$, it is concentrated in $1 \leq s \leq|A|-1$. We say that $P$ is Koszul if, for all $A$,

$$
H_{s, *}(B(P)(A), \partial)=0 \text { for } s \neq|A|-1
$$

where $\partial$ denotes the tree differential on $B(P)$.

Definition 9.43 (Koszul duals) Let $P$ be a Koszul operad in graded $k-$ modules. The Koszul dual of $P$ is the symmetric sequence $K(P)$ given by the homology of the reduced bar construction on $P$. We grade $K(P)$ according to the total degree (that is, internal degree plus tree degree) of $B(P)$ :

$$
K(P)_{r}(A)=H_{|A|-1, r+1-|A|}(B(P)(A), \partial) .
$$

Notice that $K(P)(A)$ is the kernel of the differential

$$
B(P)_{|A|-1, *}(A) \rightarrow B(P)_{|A|-2, *}(A),
$$

so there is a natural inclusion

$$
K(P) \rightarrow B(P)
$$

Proposition 9.44 Let $P$ be a Koszul operad in graded $k$-modules such that each $K(P)(A)$ is a flat $k$-module. Then the Koszul dual $K(P)$ has a natural cooperad structure.

Proof We already know from Definition 9.33 that the bar construction $B(P)$ has a cooperad structure. We get the structure for $K(P)$ by taking homology. So cocomposition maps for $K(P)$ are given by

$H\left(B(P)\left(A \cup_{a} B\right)\right) \rightarrow H(B(P)(A) \otimes B(P)(B)) \cong H(B(P)(A)) \otimes H(B(P)(B))$

where we use the flatness assumption to get the isomorphism.

We dually define the Koszul property and Koszul dual for cooperads of graded $k$-modules. 
Definition 9.45 (Koszul cooperads and Koszul duals) Let $Q$ be a reduced cooperad of graded $k$-modules. Then $Q$ is Koszul if the homology of the reduced cobar construction is concentrated in the lowest 21 tree degree. In this case, the Koszul dual of $Q$ is the symmetric sequence $K(Q)$ of graded $k-$ modules with

$$
K(Q)_{r}(A):=H_{1-|A|, r+|A|-1}\left(\Omega(Q)(A), \partial^{*}\right),
$$

where $\partial^{*}$ is the tree differential on $\Omega(Q)$. Since $K(Q)$ is the bottom homology group of $\Omega(Q)$ there is a natural surjection

$$
\Omega(Q) \rightarrow K(Q)
$$

Proposition 9.46 Let $Q$ be a Koszul cooperad of graded $k$-modules. Then the Koszul dual $K(Q)$ has a natural operad structure.

Proof The composition maps for $K(Q)$ are given by

$$
H(\Omega(Q)(A)) \otimes H(\Omega(Q)(B)) \rightarrow H(\Omega(Q)(A) \otimes \Omega(Q)(B)) \rightarrow H\left(\Omega(Q)\left(A \cup_{a} B\right) .\right.
$$

Notice that we don't need a flatness assumption here.

Fresse [7] gives various fundamental results for Koszul duality of operads and cooperads, in particular, the following.

Lemma 9.47 (Fresse, [7], Lemma 5.2.10) Let $P$ be a Koszul operad of graded $k$-modules such that the $k$-modules $P(A)$ and $K(P)(A)$ are flat. Then $K(P)$ is a Koszul cooperad and

$$
K(K(P)) \cong P
$$

as operads. Dually, let $Q$ be a Koszul cooperad of graded $k$-modules such that the modules $Q(A)$ and $K(Q)(A)$ are flat. If $Q$ is Koszul then its Koszul dual operad $K(Q)$ is also Koszul and

$$
K(K(Q)) \cong Q
$$

as cooperads.

We now give the main result of this section.

\footnotetext{
${ }^{21}$ Recall that the tree grading for the cobar construction is concentrated in negative degrees. 'Lowest' here means 'most negative'.
} 
Proposition 9.48 Let $P$ be a reduced operad in $\mathcal{T}$ or $\mathcal{S} p$ such that each object $P(A)$ is cofibrant and all homology groups $H_{*} P(A)$ and $H_{*} B(P)(A)$ are flat $k$-modules. If $H_{*} P$ is a Koszul operad then

$$
H_{*} B(P) \cong K\left(H_{*} P\right)
$$

as cooperads.

Dually, let $Q$ be a reduced cooperad in $\mathcal{S} p$ such that each object $Q(A)$ is fibrant and the homology groups $H_{*} Q(A)$ are flat $k$-modules. If $H_{*} Q$ is a Koszul cooperad then

$$
H_{*} \Omega(Q) \cong K\left(H_{*} Q\right)
$$

as operads.

Proof The cofibrancy and flatness conditions ensure that the spectral sequence of Proposition 9.39 exists for each finite set $A$ and that $H_{*} B(P)$ is a cooperad in $\operatorname{Mod}_{k}$. We have already seen that the spectral sequence has the form

$$
\left(E_{*, *}^{1}, d^{1}\right)=\left(B\left(H_{*} P\right)_{*, *}(A), \partial\right) \Longrightarrow H_{*} B(P) .
$$

Because $H_{*} P$ is Koszul, the homology of the bar construction is concentrated in the $s=|A|-1$ column. Therefore, the $E^{2}$-term is concentrated in this column and so the spectral sequence collapses. We then see that

$$
H_{r} B(P)(A) \cong E_{|A|-1, r-|A|+1}^{2} \cong H_{|A|-1, r-|A|+1}\left(B\left(H_{*} P\right)(A), \partial\right) \cong K\left(H_{*} P\right)_{r}(A)
$$

and so

$$
H_{*} B(P) \cong K\left(H_{*} P\right)
$$

as claimed. It follows that the modules $K\left(H_{*} P\right)(A)$ are flat so, by Proposition 9.44 $K\left(H_{*} P\right)$ has a cooperad structure. It remains to show that this cooperad structure agrees with that on $H_{*} B(P)$.

The first thing to notice is that the above identification of $H_{*} B(P)(A)$ with the submodule $K\left(H_{*} P\right)(A)$ on $B\left(H_{*} P\right)(A)$ is realized by an edge homomorphism of our spectral sequence. This edge homomorphism comes from applying homology to the quotient map

$$
B(P)(A) \rightarrow \bigvee_{T \in Q_{s}(A)} \underline{w}(T) \wedge P_{A}(T)
$$


where $s=|A|-1$. The key property of these maps is that they fit into commutative diagrams

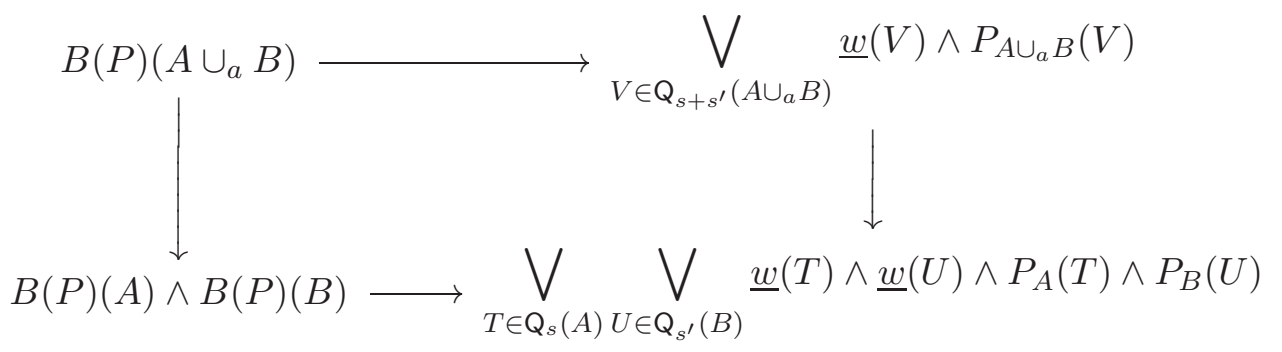

where the map on the right-hand side is built from the familiar maps

$$
\underline{w}\left(T \cup_{a} U\right) \rightarrow \underline{w}(T) \wedge \underline{w}(U)
$$

and the isomorphisms

$$
P_{A \cup_{a} B}\left(T \cup_{a} U\right) \rightarrow P_{A}(T) \otimes P_{B}(U)
$$

with terms for trees $V$ not of type $(A, B)$ mapping to the basepoint.

Taking homology of this diagram, the right-hand side map gives the cooperad structure on $B\left(H_{*} P\right)$ as described in Lemma 9.35 This shows that the edge homomorphisms of the spectral sequence identify the cooperad structure on $H_{*} B(P)$ with the restriction of that on $B\left(H_{*} P\right)$. Since the cooperad structure on $K\left(H_{*} P\right)$ is also the restriction of that on $B\left(H_{*} P\right)$, it follows that

$$
H_{*} B(P) \cong K\left(H_{*} P\right)
$$

is an isomorphism of cooperads. The dual result is proved similarly.

Remark 9.49 Proposition 9.48 extends a result of Vallette [18] for discrete operads. Recall from Remark 8.7 that he constructs the 'order complex' for an operad $P$ in Set. His main result then is that $H_{*} P$ is Koszul if and only if the homology of the order complex is concentrated in top degree. This follows immediately from our spectral sequence argument by identifying the order complex with the bar construction.

Example 9.50 We finally return to the Goodwillie derivatives of the identity functor. Recall that

$$
\partial_{*} I \cong \Omega(\underline{S})
$$

where $\underline{S}$ is the cooperad of spectra with $\underline{S}(A)=S$ for all $A$. The homology of this cooperad is given by

$$
H_{*}(\underline{S})(A)= \begin{cases}k & \text { if } *=0 \\ 0 & \text { otherwise }\end{cases}
$$


for all finite sets $A$. This is the cooperad of commutative coalgebras in the category of graded $k$-modules. Fresse shows in [7, Section 6] (by updating a result of Ginzburg and Kapranov [9]) that this cooperad is Koszul (for $k=$ $\left.\mathbb{Q}, \mathbb{F}_{p}, \mathbb{Z}\right)$ with Koszul dual given by a suspension of the Lie operad. Proposition 9.48 therefore applies and we recover the homology of the derivatives of the identity:

$$
H_{*}\left(\partial_{n} I\right)= \begin{cases}\operatorname{Lie}(n) \otimes s g n_{n} & \text { if } *=1-n ; \\ 0 & \text { otherwise. }\end{cases}
$$

Moreover, we now know that the induced operad structure on this homology of the derivatives is equal to the operad structure on the Koszul dual of the commutative cooperad, that is, the desuspended Lie structure. This completes the main goal set out in the introduction to this paper.

\subsection{Homology of modules over the derivatives of the identity}

In this final section, we use our spectral sequence to investigate the homology of the left $\partial_{*} I$-module $M_{X}$ associated to a based space $X$ as described in Remark 8.10(1). Recall that this module is given by a cobar construction:

$$
M_{X}:=\Omega\left(I, \underline{S}, \underline{\Sigma^{\infty} X}\right) .
$$

We can describe explicitly the spectral sequence for calculating $H_{*} M_{X}(2)$. The cobar construction is one-sided and we only have to consider trees for which the root has a single incoming edge. There are two 2 -labelled trees of this type with zero and one vertices respectively and a morphism between them. The $E^{1}$ term in the spectral sequence therefore only has nonzero entries in the columns $s=0$ and $s=-1$. These entries are respectively $H_{*} X$ and $H_{*}(X \wedge X) \cong H_{*} X \otimes H_{*} X$ with the differential given by the reduced diagonal $X \rightarrow X \wedge X$. The spectral sequence therefore takes the following form.

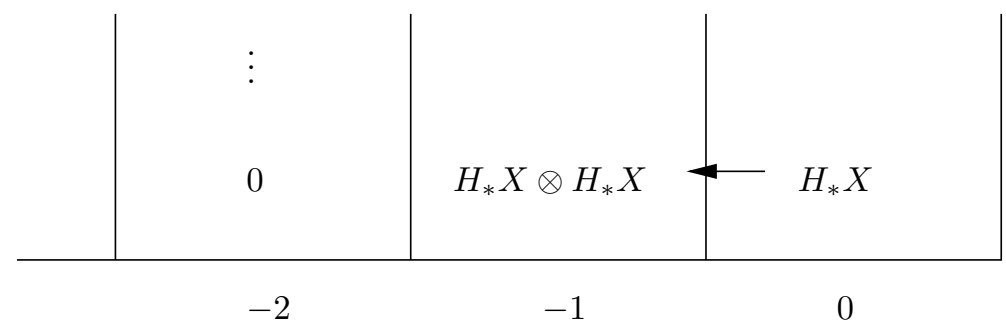

This reduces to the long exact sequence of homology determined by the cofibre sequence

$$
X \rightarrow X \wedge X \rightarrow \operatorname{hocofib}(X \rightarrow X \wedge X)
$$


which is consistent with the calculation of $M_{X}(2)$ made in Remark 8.10. Things become more interesting (and much more complicated) for $M_{X}(n)$ when $n>2$. For $n=3$ there are eight trees of interest:

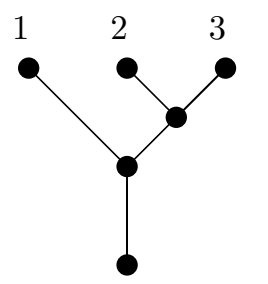

(3 labellings)

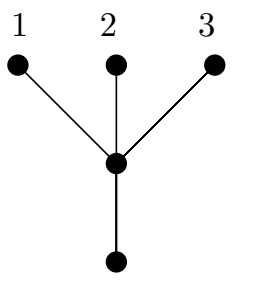

(3 labellings)

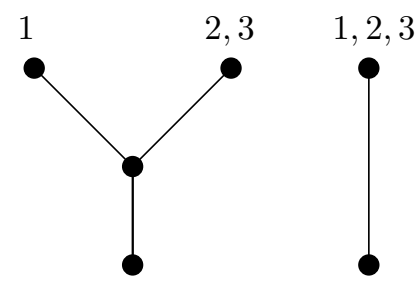

(3

and the $E^{1}$ term of the spectral sequence takes the form

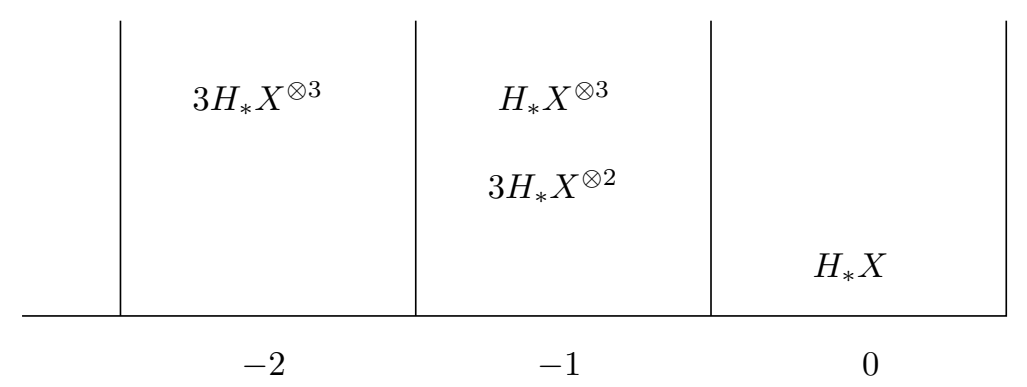

The differential $d^{1}$ is built from the reduced diagonal (between pairs of terms corresponding to bud collapse) and isomorphisms (between pairs of terms corresponding to collapse of an internal edge).

We will close the paper by looking at $X=S^{r}$, the $r$-sphere (for $r \geq 2$ ). In this situation the reduced diagonal is zero on homology and there can be no higher differentials or extensions in the spectral sequence. This allows us to calculate $H_{*} M_{S^{r}}$ with $\mathbb{Z}$ coefficients in its entirety.

Proposition 9.51 Let $S^{r}$ denote the $r$-sphere for $r \geq 2$. Then we have

$$
H_{*}\left(M_{S^{r}}\right) \cong H_{*}\left(\partial_{*} I\right) \circ H_{*}\left(\underline{S^{r}}\right)
$$

where $H_{*}\left(\underline{S^{r}}\right)$ is the symmetric sequence with

$$
H_{*}\left(\underline{S^{r}}\right)(n)= \begin{cases}\mathbb{Z} & \text { if } *=r \\ 0 & \text { otherwise }\end{cases}
$$

The left action of $H_{*}\left(\partial_{*} I\right)$ on $H_{*}\left(M_{S^{r}}\right)$ is given by the operad structure on $H_{*}\left(\partial_{*} I\right)$. 
Proof The $E^{1}$ term of the spectral sequence for the homology of $M_{X}$ is in this case the algebraic cobar construction

$$
\Omega\left(I, H_{*}(\underline{S}), H_{*}\left(\underline{S^{r}}\right)\right) .
$$

The coaction of $H_{*}(\underline{S})$ on $H_{*}\left(\underline{S^{r}}\right)$ is trivial in the sense that the only nonzero cocomposition maps are

$$
H_{*}\left(\underline{S^{r}}\right)(n) \rightarrow H_{*}(\underline{S})(1) \otimes H_{*}\left(\underline{S^{r}}\right)(n) .
$$

This is equivalent to saying that

$$
H_{*}\left(\underline{S^{r}}\right) \cong I \circ H_{*}\left(\underline{S^{r}}\right)
$$

as left $H_{*}(\underline{S})$-comodules, where the coaction of $H_{*}(\underline{S})$ on the right-hand side is via the coaugmentation action on $I$. It follows that the $E^{1}$ term of our spectral sequence can be written

$$
\Omega\left(I, H_{*}(\underline{S}), I \circ H_{*}\left(\underline{S^{r}}\right)\right) \cong \Omega\left(I, H_{*}(\underline{S}), I\right) \circ H_{*}\left(\underline{S^{r}}\right)
$$

where the differential on the right-hand side comes solely from the cobar construction and not from $H_{*}\left(\underline{S^{r}}\right)$. This isomorphism can be seen by working through the definition of the algebraic bar construction in this case.

It now follows that the $E^{2}$ term of our spectral sequence is given by

$$
H_{*}\left(\partial_{*} I\right) \circ H_{*}\left(\underline{S^{r}}\right) .
$$

In the $E^{2}$ term for calculating $H_{*} M_{S^{r}}(n)$, we only have nonzero entries in bidegrees $(-k, r(k+1))$ for integers $k \geq 0$. Since $r \geq 2$ there can be no further differentials or extensions and so we see that

$$
H_{*}\left(M_{S^{r}}\right) \cong H_{*}\left(\partial_{*} I\right) \circ H_{*}\left(\underline{S^{r}}\right) .
$$

The proof of Proposition 9.48 extends to show that the left action of $H_{*}\left(\partial_{*} I\right)$ is as claimed.

Remark 9.52 The functor $P \circ-$ from symmetric sequences to left $P$-modules is left adjoint to the forgetful functor and so can rightfully be called the free left $P$-module functor. Hence the homology of $M_{S^{r}}$ is the free left $P$-module on $H_{*}\left(\underline{S^{r}}\right)$.

Explicitly, there is a generator $x_{A}$ in $H_{r}\left(M_{S^{r}}\right)(A)$ for each finite set $A$. The entire homology group $H_{*}\left(M_{S^{r}}\right)(A)$ then has a basis given by all possible iterated brackets of the form

$$
\left[\ldots\left[\left[x_{A_{1}}, x_{A_{2}}\right], x_{A_{3}}\right] \ldots, x_{A_{k}}\right]
$$

where $A_{1}, \ldots, A_{k}$ is a partition of $A$ into nonempty finite subsets, and $[-,-]$ is a Lie bracket of degree -1 . This Lie bracket also represents the action of $H_{*}\left(\partial_{*} I\right)$ on $H_{*}\left(M_{S^{r}}\right)$. 


\section{References}

[1] Greg Arone, Mark Mahowald, The Goodwillie tower of the identity functor and the unstable periodic homotopy of spheres, Invent. Math. 135 (1999) 743-788 MathReview

[2] Clemens Berger, Ieke Moerdijk, Axiomatic homotopy theory for operads, Comment. Math. Helv. 78 (2003) 805-831 MathReview

[3] Francis Borceux, Handbook of categorical algebra. 2. categories and structures, Encyclopedia of Mathematics and its Applications 51, Cambridge University Press, Cambridge (1994), MathReview

[4] Michael Ching, Arboreal objects and operads, in preparation

[5] Michael Ching, A note on the composition product of symmetric sequences, arXiv:math.CT/0510490

[6] A D Elmendorf, I Kriz, MA Mandell, J P May, Rings, modules, and algebras in stable homotopy theory, with an appendix by M Cole, Mathematical Surveys and Monographs 47, American Mathematical Society, Providence, RI (1997), MathReview

[7] Benoit Fresse, Koszul duality of operads and homology of partition posets, from: "Homotopy theory and its applications (Evanston, 2002)", Contemp. Math. 346, Amer. Math. Soc. Providence, RI (2004) 115-215 MathReview

[8] Ezra Getzler, J D S Jones, Operads, homotopy algebra and iterated integrals for double loop spaces, arXiv:hep-th/9403055

[9] Victor Ginzburg, Mikhail Kapranov, Koszul duality for operads, Duke Math. J. 76 (1994) 203-272 MathReview

[10] Thomas G Goodwillie, Calculus. I. The first derivative of pseudoisotopy theory, $K$-Theory 4 (1990) 1-27 MathReview

[11] Thomas G Goodwillie, Calculus. II. Analytic functors, $K$-Theory 5 (1991/92) 295-332 MathReview

[12] Thomas G Goodwillie, Calculus. III. Taylor series, Geom. Topol. 7 (2003) 645-711 MathReview

[13] Mark Hovey, Model categories, Mathematical Surveys and Monographs 63, American Mathematical Society, Providence, RI (1999) MathReview

[14] L G Lewis, Jr, J P May, M Steinberger, J E McClure, Equivariant stable homotopy theory, Lecture Notes in Mathematics 1213, Springer-Verlag, Berlin (1986) MathReview

[15] Saunders MacLane, Categories for the working mathematician, Graduate Texts in Mathematics 5, Springer-Verlag, New York (1971), MathReview

[16] Martin Markl, Steve Shnider, Jim Stasheff, Operads in algebra, topology and physics, Mathematical Surveys and Monographs 96, American Mathematical Society, Providence, RI (2002) MathReview 
[17] Paolo Salvatore, Configuration operads, minimal models and rational curves, $\mathrm{PhD}$ thesis, University of Oxford (1998)

[18] Bruno Vallette, Homology of generalized partition posets, (2004)

[19] R M Vogt, Cofibrant operads and universal $E_{\infty}$ operads, Topology Appl. 133 (2003) 69-87 MathReview 